\title{
Structural and electronic properties
}

\author{
of swift heavy ion tracks
}

\section{in amorphous carbon}

\author{
Dissertation \\ zur Erlangung des Doktorgrades der \\ Mathematisch-Naturwissenschaftlichen Fakultäten \\ der Georg-August-Universität zu Göttingen
}

\author{
vorgelegt von \\ Daniel Schwen \\ aus Hannover
}

Göttingen 2007 
D7

Referent: Prof. Hans Hofsäss

Korreferent: PD Dr. Alexander Hartmann

Tag der mndlichen Prüfung: 14.2.2007 


\section{Contents}

1 Introduction $\quad 1$

1.1 Motivation . . . . . . . . . . . . . . . . . . . 1

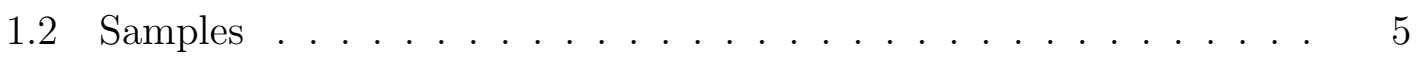

1.2.1 Tetrahedral amorphous carbon films . . . . . . . . 5

1.2.2 Fullerene films . . . . . . . . . . . . . . . . . . . 6

1.3 Ion track formation in solids . . . . . . . . . . . . . . 7

1.4 Electrical transport . . . . . . . . . . . . . . . . . . . . . . . . 12

1.4.1 Electronic structure . . . . . . . . . . . . . . . . 12

1.4.2 Conduction mechanisms in amorphous semiconductors . . . 13

1.4.3 Frenkel-Poole conduction . . . . . . . . . . . . . . 15

1.4.4 Variable range hopping . . . . . . . . . . . . . . . 16

1.4.5 Current distribution . . . . . . . . . . . . 18

2 Computational methods and results 23

2.1 Molecular dynamics simulations . . . . . . . . . . . . . . . . 23

2.1 .1 Interatomic potentials . . . . . . . . . . . . . 24

2.1.2 Implementation details . . . . . . . . . . . . . . . . . 26

2.1.3 Sample preparation . . . . . . . . . . . . . . . . . . . 27

2.1.4 Simulation setup . . . . . . . . . . . . . . . . . . . . . . . . . . . 30

2.1.5 Results . . . . . . . . . . . . . . . . . . . . . . 32

2.2 Density of states calculations . . . . . . . . . . . . . . 43

2.3 TEM image simulation . . . . . . . . . . . . . . . . . . . . . 46

2.3.1 Averaged potential image simulation . . . . . . . . . . . 50

2.3.2 High resolution image simulation . . . . . . . . . . . 52

3 Experimental results $\quad \mathbf{5 5}$

3.1 Atomic force microscopy . . . . . . . . . . . . . . . . . 55

3.1.1 Conducting atomic force microscopy . . . . . . . . . . . 57

3.1.2 Statistical hillock-height analysis . . . . . . . . . . . 58

3.2 Scanning tunneling microscopy . . . . . . . . . . . . . . . . . . 61

3.3 Current-voltage spectroscopy . . . . . . . . . . . . . . . 67 
3.3.1 Polycarbonate masking . . . . . . . . . . . . . . . 72

3.4 Plane view TEM analysis . . . . . . . . . . . . . . . . . . . 75

3.4.1 Sample preparation . . . . . . . . . . . . . 77

3.5 Irradiated fullerene films . . . . . . . . . . . . . . . . . . . 79

3.5.1 Thermal desorption . . . . . . . . . . . . . 80

3.5.2 Raman measurements . . . . . . . . . . . . . . . 82

3.5.3 AFM measurements . . . . . . . . . . . . . 84

$\begin{array}{llr}4 & \text { Discussion and conclusions } & 87\end{array}$

A Current Distribution $\quad 95$

B Publications $\quad 99$

B.1 List of publications . . . . . . . . . . . . . . . . . . . 99

B.2 Conference contributions . . . . . . . . . . . . . . 101

$\begin{array}{ll}\text { C List of Abbreviations } & 103\end{array}$

$\begin{array}{ll}\text { List of Figures } & 105\end{array}$

$\begin{array}{ll}\text { Bibliography } & 109\end{array}$ 


\section{Chapter 1}

\section{Introduction}

\subsection{Motivation}

It has long been known, that swift ions traveling through solids leave a trail of structural modifications along their trajectories. The study of ion tracks in solids was sparked in the late fifties when an increase of bulk etachability in radiation damaged lithium fluoride was discovered by Young et al. in 1958 [You58]. They already proposed the concept of a chain of defects created by each fission fragment. And only shortly after in 1959 Silk et al. detected individual tracks in mica on transmission electron micrographs [Sil59].

Tracks formed by fission fragments can be used for geological dating [Fle64a]. The individual track morphology allows to deduce the stopping powers of the fission fragments [HN05] and a pressure and temperature history ultimately yielding an understanding of lithospheric movements, crustal cooling, and uplift [Hur91].

Lunar and interplanetary materials such as cosmic dust or meteorites are susceptible to cosmic ray induced track formation [Ree83]. Ion tracks have been detected in interplanetary dust particles [Bra84], and ion bombardment is governing the morphological evolution of stellar carbon dust [Bus93]. The latter is detectable spectroscopically in the infrared and UV regions [Mar90, Men97] and possesses spectral features allowing for a quantization of carbon allotropes in the dust particles. The understanding of individual ion impact processes could lead to the identification of the timescales for the morphological changes, allowing to infer age or initial composition of the dust. The tracks detected in lunar rocks brought back to earth on 
the Apollo missions [Cro74] give insight into cosmic ray exposure times and, thus, changes of the lunar surface and a measure of cosmic ray activity.

Materials that exhibit tracks, which through etching can be made visible under an optical microscope, such as polycarbonate, mica, and cellulose nitrate, were investigated for their suitability as track detectors [Fle64b]. These detectors are cheap, producible in almost any shape or size and possess a high detection efficiency, energy specificity, and spatial resolution, arguably shifting the complexity from detection to detector readout.

Ion accelerators provide a controllable setup to study the effects of a variety of ion species at well defined energies on a wide range of materials. Ion tracks manifest in a variety of morphological changes, depending on the matrix material. Two regimes have to be discerned, the bulk region far away from entry or exit surfaces, and the surface near region. Due to the short timescales of the processes involved in track formation, material transport parallel to the ion trajectory plays no role far away from the surface. Flows in both directions cancel each other out for symmetry reasons. Amorphization can be observed in crystalline materials such as mica or lithium fluoride. In already amorphous materials a density reduction along the ion trajectories is expected [Kra03]. Certain materials even form hollow tubes along the trajectories [Bol06].

In polymers a change of the chemical composition due to chain breaking was observed early [Fle67, Fer93]. Each breaking point introduces chemically active sites, which can be attacked by a suitable etchant, allowing to create hollow channels at the sites of the latent tracks. Polymer foil stacks are commonly used as track detectors [Lan05]. Made visible through etching the tracks are counted under an optical microscope. The penetration depth in the stack allows the calculation of the ion energies. When using polymer foils with thicknesses well below the ion ranges, porous membranes can be created through the etching process, which can serve as ultrafine filters. These filters have applications for gas separation [Sud01] and ultrafiltration [Ape03] of bio molecules. Preliminary experiments for DNA detection have been performed, and techniques for the sequencing of single DNA molecules have been proposed [Mar04]. Through electro-chemical deposition the etched pores can be filled, creating nanowires with tunable diameters [Mol01, Dob01]. 
Near the surface, however, the aforementioned symmetry is broken, and material transport causes surface effects such as swelling [Tra00] or material ejection and cratering [Bri02b]. In the case of nickel oxide surface tension forms tiny spheres from the material ejected at the entry and exit points of the ion trajectory.

To achieve a direct functionalization of the ion tracks, which exceeds etchability or contrast changes in transmission electron micrographs, matrix materials which exhibit distinct physical properties in the unmodified and ion irradiated phases are needed. One such material is carbon. As a bulk material at standard temperature and pressure it coexists in the stable threefold coordinated graphitic phase and the metastable fourfold coordinated diamond phase. The different coordination numbers are achieved by the hybridization, the orbital mixing of $\mathrm{s}$ and $\mathrm{p}$ orbitals at each atomic site. Mixing of the s orbital with two of the three p orbitals is called the $\mathrm{sp}^{2}$ state, while the mixing of the s orbital with all three $\mathrm{p}$ orbitals is called the $\mathrm{sp}^{3}$ state. The $\mathrm{sp}^{2}$ state favors a planar bond distribution, while the $\mathrm{sp}^{3}$ state favors a tetrahedral spacefilling bond angle distribution.

The planar bond distribution in graphite gives rise to the sheet-like structure of the material. Each graphene sheet is made up of a two-dimensional hexagonal grid of strongly bound carbon atoms, while the inter-sheet bonding is dominated by weak van der Waals dispersion forces. The $\mathrm{p}$ orbitals not participating in the $\mathrm{sp}^{2}$ hybridized state form a network of delocalized $\pi$-bonds, making graphite a semimetal and an electrical conductor parallel to the graphene sheet directions.

The tetrahedral spacefilling bond structure of diamond makes it the hardest known material. Absence of $\pi$-bonds and the huge energy difference between the binding and anti-binding $\mathrm{sp}^{3}$ states make diamond a semiconductor with a wide band-gap of $5.45 \mathrm{eV}$ which is practically an electrical insulator at room temperature.

As the $\mathrm{sp}^{2}$ and $\mathrm{sp}^{3}$ bond states are almost isoenergetic, any mixture of $\mathrm{sp}^{2}$ and $\mathrm{sp}^{3}$ hybridized carbon atoms can form a stable amorphous network. The properties of this amorphous carbon (a-C) material are strongly dependent on the $\mathrm{sp}^{2}: \mathrm{sp}^{3}$ ratio. Predominantly $\mathrm{sp}^{3}$ bound materials, also called tetrahedrally bound amorphous carbon (see 1.2.1), resemble diamond in that they are also optically tansparent and electrically insulating. While the properties of predominantly $\mathrm{sp}^{2}$ bound amorphous carbon, abundant in soot, or glassy carbon, resemble those of graphite, being opaque and electrically conducting. 
While the transition from diamond to graphite would provide the most drastic change in physical properties, to this date no formation of continuous ion tracks has been reported in either material, and part of this work deals with this absence of tracks. Amorphous carbon materials, however, undergo morphological changes under swift heavy ion irradiation resulting in a modification of the $\mathrm{sp}^{2}: \mathrm{sp}^{3}$ ratio along the ion tracks [Kra01].

In the diamond-like tetrahedrally bound amorphous carbon (ta-C) a change to a more graphitic form has been observed. This effect could be used to selectively modify the electrical properties on a nanometer scale. The resulting tracks constitute wires which are extremely thin while having a high aspect ratio, suggesting a possible application as field emission cathodes [Sch04].

This work aims to combine experimental and computational investigations to shed light on the morphology, geometry and electronic properties of ion tracks in amorphous carbon. The introductory chapter continues with a brief description of the carbon sample materials used in the irradiations and the basic concepts of track formation in solids and closes with a discussion of the basic electrical transport mechanisms in amorphous solids relevant for the studied materials.

Chapter 2 encompasses all computational investigations of this work. It begins with a short introduction to molecular dynamics simulations with a discussion of the interatomic potentials used and some relevant implementation details. The setups of the simulations are described, with emphasis on the non-trivial generation of an amorphous carbon sample. The results of the simulations are discussed and number comparisons of the track formation processes in amorphous carbon, graphite, and diamond is presented. Some of the results in this chapter are published [Sch06]. The large scale simulations investigating surface hillocks induced by swift heavy ion irradiation are the largest performed to date by several orders of magnitude to the best of the author's knowledge. The relation of projectile ion energy loss and hillock dimensions is studied. The molecular dynamic simulation results of ion tracks were taken as the basis for further computational experiments. Electronic structure calculations were performed on small sub-samples and virtual transmission electron microscopy (TEM) micrographs were calculated to facilitate comparisons to experimental data. 
The experimental results are presented in chapter 3. Atomic force microscopy (AFM) measurements were performed to determine the surface morphology of the irradiated samples. Simultaneous conducting AFM measurements to map the electrical properties provide additional information on the local ion induced modifications. Scanning tunneling microscopy (STM) and spectroscopy (STS) was used to investigate the electronic structure in surface-near regions. Supplementary macroscopic temperature-dependent current-voltage measurements on large track ensembles were performed to shed light on the electrical transport properties. An I(V,T) spectroscopy setup was developed and assembled to perform electrical measurements at temperatures down to $15 \mathrm{~K}$. To gain insight into the material densty distribution inside the tracks samples were prepared for TEM. Fullerene $\mathrm{C}_{60}$ films were irradiated as a complementary system, providing insight into the radial damage distribution.

The concluding remarks in chapter 4 discuss the relation of computational and experimental results. In particular a justification for the treatment of ion tracks as a thermal spike process is given, and a linear scaling modell for the energy transfer from the electronic system to the lattice is proposed.

\subsection{Samples}

\subsubsection{Tetrahedral amorphous carbon films}

Tetrahedral amorphous carbon (ta-C) is a carbon material with no long-range ordering with a high ratio of $\mathrm{sp}^{3}: \mathrm{sp}^{2}$ hybridized carbon atoms. The high $\mathrm{sp}^{3}$ content determines the properties of ta-C, such as optical transparency and high electrical resistivity.

The samples used in this study were prepared using mass selected ion beam deposition (MSIBD). The source material, a $\mathrm{CO}_{2}$ and argon gas mixture, is broken down and ionized in a hot filament and hollow cathode Sidenius type plasma ionsource. The extracted and accelerated ions are mass-separated with isotopic resolution passing a sector magnet. At the substrate the ions are decelerated to the desired deposition energy. Several ion-optical elements ensure beam convergence, and a beam-sweep guarantees a homogeneous deposition across an area of about 
half a square centimeter on the substrate. The amount of deposited material is determined by beam-current integration, assuming that only singly ionized carbon atoms pass the mass separation magnet.

Mass selection with isotope resolution, deposition in ultra high vacuum (UHV) conditions, and well defined parameters such as temperature and ion energy make MISBD an ideal tool to study ion beam deposition processes and grow films with well defined properties under exactly reproducible consitions. All films examined in this work were grown at room temperature at an ion beam energy of $100 \mathrm{eV}$, which was found to maximize the $\mathrm{sp}^{3}$ content of the films at about $70 \%$ [Ron96]. The growth process takes place in the sub-surface implantation regime and is well described by the cylindrical thermal spike model [Hof98].

Prior to deposition all substrates were sputter cleaned using $15 \mathrm{mC}$ of a $1 \mathrm{keV}^{40} \mathrm{Ar}^{+}$ ion beam to remove the native oxide layer. Details of the deposition process are described by Hofsäss et al. [Hof93] and Ronning [Ron03].

\subsubsection{Fullerene films}

As a complementary carbon based thin film system fullerene $\mathrm{C}_{60}$ [Kro85, Krä90] was chosen. The films were deposited using an resistively heated Knudsen cell consisting of a molybdenum crucible heated by an attached UHV button heater. As a source material commercially available $\mathrm{C}_{60}$ (MerCorp) was used, the substrate material was silicon, dipped into hydrofluoric acid to remove the native oxide layer. The deposition rate of the fullerens was kept below $10 \mathrm{pm} / \mathrm{s}$, with the substrates at room temperature. UHV chamber base pressure was $10^{-10} \mathrm{mbar}$, rising to $10^{-8} \mathrm{mbar}$ during deposition.

Upon deposition $\mathrm{C}_{60}$ forms either hexagonal close-packed (hcp) structures, the slightly more stable [Sko05] face-centered cubic (fcc) structure, or a mixture of the two, which only differ in stacking order. Heteroepitaxy is possible on lattice matching substrates such as germanium sulfide (GeS) (001) cleaved surfaces. On non-matching substrates the films exhibit a columnar structure. With a film density of $1.67(2) \mathrm{g} / \mathrm{cm}^{3}$ [Kol95] the material is $25 \%$ less dense than graphite. The $\mathrm{C}_{60}$ molecules in those films are only bound by van der Waals forces and, above a transition temperature of $260 \mathrm{~K}$, can rotate freely around their center points. This 
weak bonding also explains the low sublimation temperature of $627 \mathrm{~K}$ and solubility in various organic compounds such as 1,2,4-trichlorbenzene.

\subsection{Ion track formation in solids}

A swift heavy ion can interact with matter through four mechanisms. Two of those mechanisms, dominating in the relativistic regime, emission of Cherenkov radiation or coherent bremsstrahlung lead to the direct creation of photons, which carry away the energy from the ion trajectory. Their contributions to the formation of ion tracks can therefore be neglected. The remaining two mechanisms are scattering on target nuclei and scattering on target electrons, each process dominating in a particular energy range. These energy ranges are determined by atomic numbers and masses of projectile ions and target atoms, and the density of the target material. The experimentally investigated target material was ${ }^{12} \mathrm{C}$ at densities in between $1.67 \mathrm{~g} / \mathrm{cm}^{3}$ for fullerene films and about $3 \mathrm{~g} / \mathrm{cm}^{3}$ for amorphous carbon.

At projectile energies below about $10^{4} \mathrm{keV}$ (fig. 1.1) a considerable amount of energy is transferred directly to the lattice of the target material through scattering of the swift heavy ion at the target nuclei. This energy loss mechanism is called nuclear stopping $S_{n}$ and results in the direct creation of lattice vibrations or (in crystralline materials) phonons. For the projectile ion species used in this work this scattering process yields a maximal energy loss contribution around $500 \mathrm{keV}$.

Above this energy the interaction of the projectile ions with the target electrons, the so called electronic stopping $S_{e}$, becomes the significant contribution. Its onset is correlated to the increase of ion-electron interaction time, when ion velocity becomes comparable to the Fermi velocity of the electrons in the target material. At the same time the interaction time between the fast projectiles and the quasi-static nuclei diminishes, and the nuclear stopping is reduced. The electronic stopping power maximum $S_{e, B}$, the Bragg peak, is located at around $1 \mathrm{GeV}$ projectile energy. Projectile energies beyond the Bragg preak and thus higher projectile velocities lead to a reduction of the ion-electron interaction time and a reduction of the electronic stopping power.

A specific stopping power $S_{e, 1}<S_{e, B}$ can be found on both sides of the Bragg peak, at high $E_{h}$ and at low $E_{l}$ projectile energies. Although equal in linear stopping power 


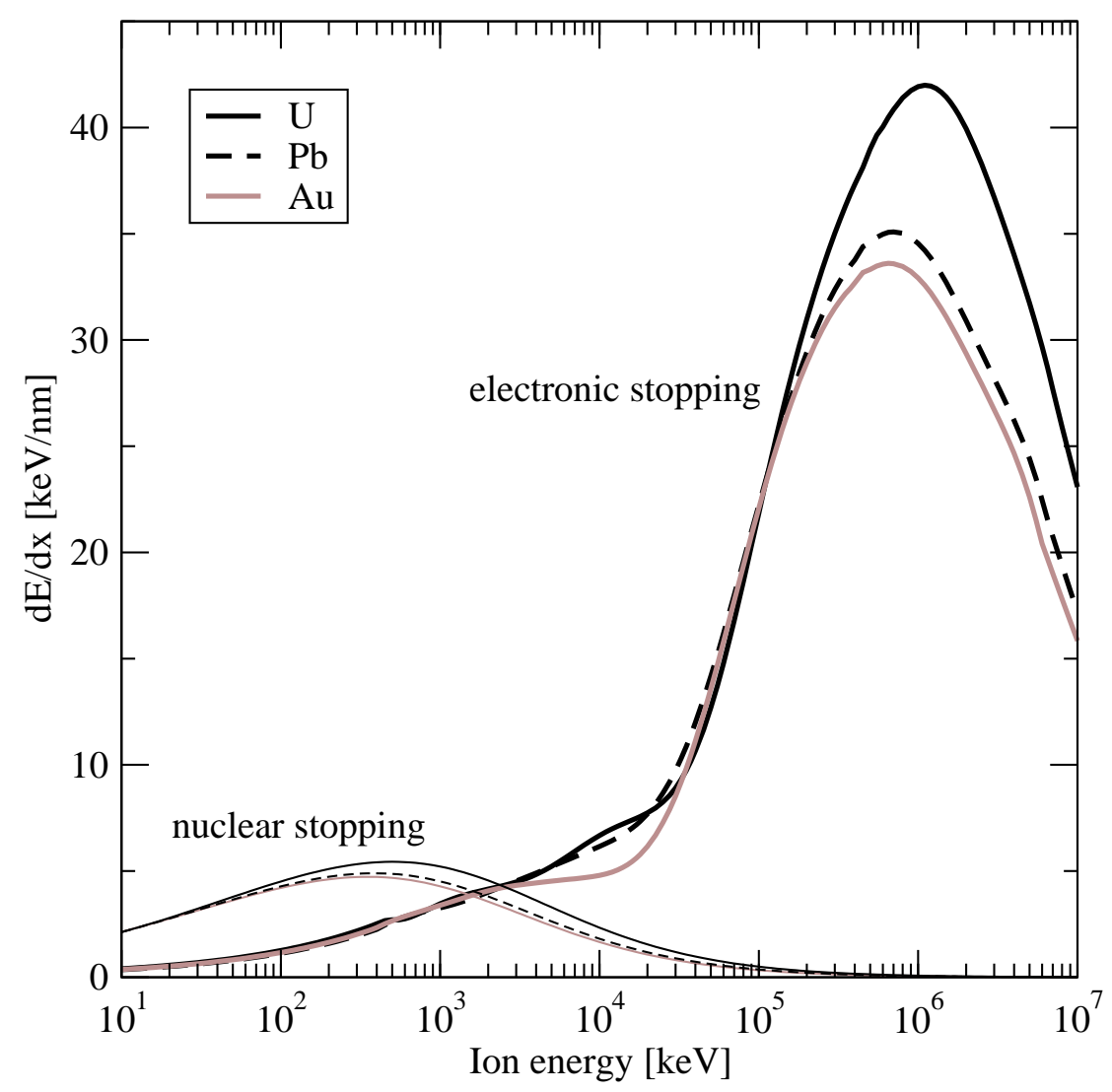

Figure 1.1: Stopping powers of select ions in ta-C in the range of $10 \mathrm{keV}$ to $10 \mathrm{GeV}$, calculated using the Monte-Carlo software SRIM2003 [Zie85]. All irradiations were performed close to the electronic stopping power maximum.

$d E / d x$, the deposited energy density varies with the projectile velocity [Kat69, Wal86]. This dependence is called velocity effect and leads to higher energy densities for low velocities, and spread-out excitations for high velocities (eq. 1.1).

All irradiations were performed at room temperature with ions impinging perpendicular to the film surface (fig. 1.2) (fig 1.2). The swift heavy ion passes the film within $\approx 1 / 100$ fs (fig. 1.3) at a speed of about $5 \% c\left(1 \mathrm{GeV}^{238} \mathrm{U}\right)$. At typical electronic energy losses of about $30-40 \mathrm{keV} / \mathrm{nm}$, the projectile ion creates a trail of electronic excitations. The electronic temperature during this early phase of track formation has been measured by spectroscopic analysis of Auger electrons ejected from the tracks [Sch99, Sch00], yielding values of over $5 \cdot 10^{4} \mathrm{~K}$, depending on the 


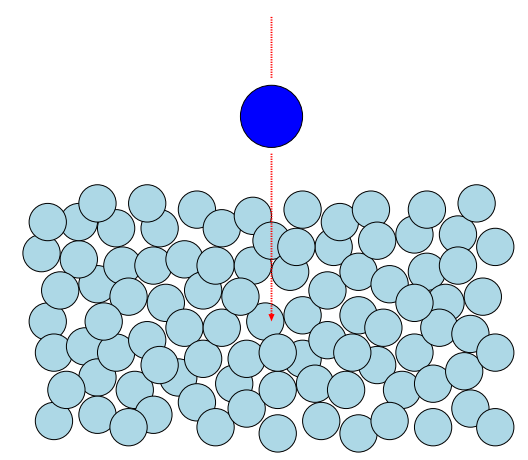

Figure 1.2: Ta-C film with a density of about $\approx 3 \mathrm{~g} / \mathrm{cm}^{3}$, irradiation perpendicular to film surface.

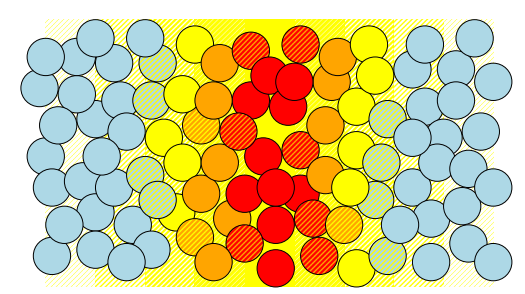

Figure 1.4: Electronic excitations couple to the lattice, heating it up, energy is available for atomic rearrangement.

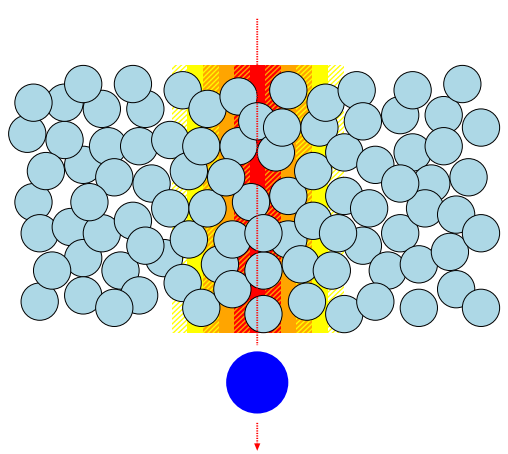

Figure 1.3: Swift heavy ion passes within $\approx 1 / 100 \mathrm{fs}$, leaving a trail of high energetic electronic excitations.

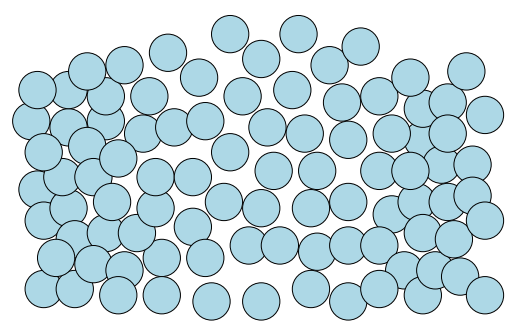

Figure 1.5: Density is reduced, $\mathrm{sp}^{2}$ fraction increased, heat dissipates within ps. 
projectile charge. The spatial extent of the excited region can be described by the Bohr adiabatic radius

$$
a_{\mathrm{ad}}=\frac{v}{\omega_{0}}
$$

with $v$ being the projectile ion velocity and $\omega_{0}$ the plasmon frequency of the target material. For a $1 \mathrm{GeV}^{235} \mathrm{U}$ ion at $v=2.45 \cdot 10^{7} \mathrm{~m} / \mathrm{s}$ in amorphous carbon with a plasmon energy of $\approx 30 \mathrm{eV}\left(\omega_{0}=4.56 \cdot 10^{16} \mathrm{~s}^{-1}\right)$ eq. 1.1 yields a radius $a_{\text {ad }}$ of about $5.4 \AA$.

Within the next few femtoseconds [Wan94, Tou02, Rot04], the electronic excitations couple to the lattice. During this time the excitations can diffuse away from the track center, leading to a further increase of the excited track radius. Coupling mechanisms include electron-phonon interaction or electrostatic effects such as coulomb-explosion or long-lived repulsive states.

A moving electron constitutes a perturbation of the interatomic potential instantly modulating the forces acting on the atoms it passes. With the force change the atomic equilibrium coordinates shifts, accelerating the atom. In a periodic lattice this leads to a displacement wave following the electron, called polaron.

Local excitation of the electronic system can lead to a charge separation. Electrons are displaced from the track region leaving behind an ionized region. If the recombination time is sufficiently long, the electrostatic potential energy of the ionized lattice can be transformed to kinetic energy and deformation in a process called coulomb explosion.

This transfer from the electronic system to the lattice causes a local heating (fig. 1.4). For about a picosecond the temperature inside the track is above the melting temperature $T_{\text {melt }}$ of the matrix material. This leads to a pressure increase, expansion, and a density reduction. With density the amorphous carbon material must also change its coordination, leading to an increase of the $\mathrm{sp}^{2}$-fraction. As the heat dissipates, the track freezes and the density change is conserved (fig. 1.5).

Not all energy deposited by the swift heavy ion into the electronic system is available for atomic rearrangement. Some energy gets carried away perpendicularly from the track core by high energetic $\delta$ electrons. Convoy electrons [Cro70] receive a high forward momentum, travel with the potential wake of the projectile and are displaced into the substrate depositing their energy far from the track. Depending 


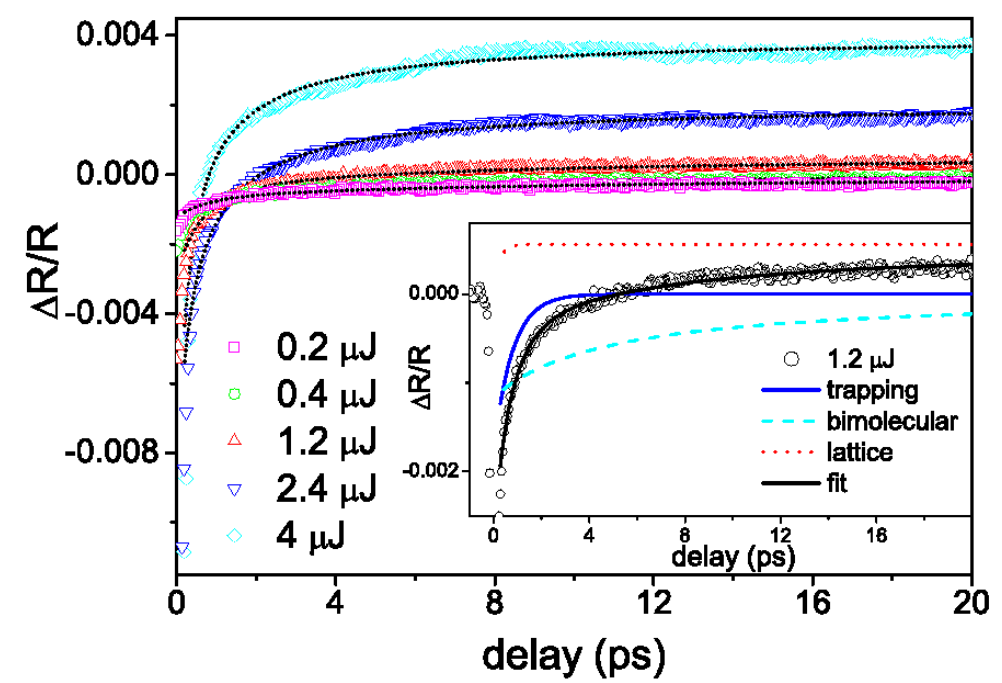

Figure 1.6: Differential reflectivity data as a function of pump probe delay (from [Car07]). Each curve corresponds to a different pump energy. As shown in the inset on the $1.2 \mu \mathrm{J}$ measurement, the data is fit with a three component model considering carrier trapping (solid line), direct electron-hole recombination (dashed line), and lattice heating (dotted line).

on the matrix material, the excited electrons can also recombine radiatively. This has been observed as ionoluminescence in diamond [Man01].

In a recent collaboration with Carpene et al. [Car07] (in preparation) the carrier dynamics in ta-C was investigated by means of femtosecond reflectivity measurements. The primary pulse of the third harmonic of a titanium sapphire (Ti:sapphire) laser with $4.65 \mathrm{eV}$, well above the optical gap of ta-C, excites the electronic system, creating free carriers. The free carrier concentration determines the sample reflectivity as described by the Drude model for the complex dielectric function [Oth02]. The reflectivity is probed using a secondary pulse of Ti:sapphire laser light at $1.55 \mathrm{eV}$. The time delay between primary and secondary pulse can be tuned with femtosecond accuracy by changing signal path length using a Fabry-Pérot setup. Repeated cycles of excitation and probing with varying time delays allow measuring the time evolution of the free carrier concentration. The pulse duration of about 60 fs determines the temporal resolution. 


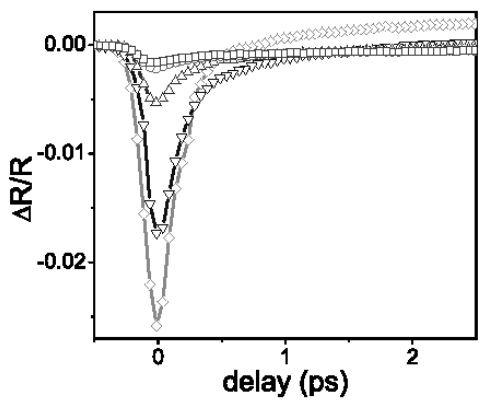

Figure 1.7: Detail of the $\Delta R / R$ time evolution. Legend see fig. 1.6.

Primary pulse energy densities of about 0.5 to $5 \mathrm{eV} / \mathrm{nm}^{3}$, corresponding to energies between $0.2 \mu \mathrm{J}$ and $4.0 \mu \mathrm{J}$ across a spot $500 \mu \mathrm{m}$ in diameter with an approximate penetration depth of $25 \mathrm{~nm}$, were used in the experiment. These energy densities are well below those in a swift heavy ion track, which are of the order of a few $100 \mathrm{eV} / \mathrm{nm}^{3}$. Caution has to be exercised in applying the results to track formation processes. Nonetheless, within the energy density window investigated most energy is transferred from the electronic system to the lattice within the first $0.25 \mathrm{ps}$, regardless of the energy density (fig. 1.7). The data is fit with a three component model considering carrier trapping, direct electron-hole recombination, and lattice heating (fig. 1.6) yielding information on electron-phonon relaxation time $\tau_{e p}$, which was determined to be $220 \pm 35 \mathrm{fs}$, and the trap state density, which was determined to be in between $10^{19} \mathrm{~cm}^{-3}$ and $10^{20} \mathrm{~cm}^{-3}$.

\subsection{Electrical transport}

This chapter presents an overview of transport mechanisms in amorphous semiconductors and discusses their relevance with respect ta-C.

\subsubsection{Electronic structure}

The electronic structure describes the permitted energies of electrons in matter. In isolated atoms these permitted energies are given by the bound states of the electrons and are characterized by a set of quantum numbers. Identical atoms possess identical sets of states and, thus, energy levels.

For atoms in close proximity the Pauli exclusion principle forbids more than two electrons of opposing spin to share energetically identical states, leading to a splitting of energy levels. If the separation of the shifted energy level becomes lower than the electron energy fluctuations due to phonon absorption and emission, or - at low temperatures - the energy sharpness given by the uncertainty principle, then the individual levels form continuous energy bands. Of those bands mainly 
the valence band, the highest occupied band at zero temperature, and the conduction band, the lowest unoccupied band, determine the electronic properties of a solid. All other bands are energetically to far from the Fermi level to participate in electron transport.

The density of states (DOS) at a particular energy is ultimately determind by chemical composition and atomic structure of a material. In amorphous materials the DOS is largely determined by the short range order. Local structure and the closest neighbors determine the energy levels which combined make up the electronic structure.

The surface atoms are expected to exhibit a local DOS different from the bulk atoms. Due to a reduced number of next neighbors, the surface atoms are undercoordinated. STM measurements confirm the atomic smoothness of the films. Thus, in good approximation one half space at each surface atom is empty, inhibiting the formation of $\mathrm{sp}^{3}$-coordinated sites. Surface sensitive DOS measurements are expected to yield results skewed towards a higher $\mathrm{sp}^{2}$ fraction.

\subsubsection{Conduction mechanisms in amorphous semiconductors}

Deviations from the ideal $\mathrm{sp}^{2}$ or $\mathrm{sp}^{3}$ site configurations result in weakend bonds. These bonds give rise to elevated binding energy levels slightly above the valence band edge, and anti-binding energy levels slightly below the conduction band edge. These perturbed states constitute the band tails, which generate a DOS fall-off reaching into the forbidden gap. Depending on their volume density these tail states are either delocalized or localized [And58, Kra93], giving rise to a sharp transition energy between localized and continuum states, called mobility edge [Coh69].

Two classes of conduction mechanisms in amorphous solids can be identified (fig. 1.8). In the gap conduction can occur through tunneling processes (a,b) in between localized states. Carriers in the band-edge regime can be thermally excited into the conduction band (c), temporarily contribute to the electrical conduction, and quickly be trapped in localized states (d) below the conduction band due to inelestic enrgy losses in the solid. Analog mechanisms as for electrons apply for hole-carriers. 

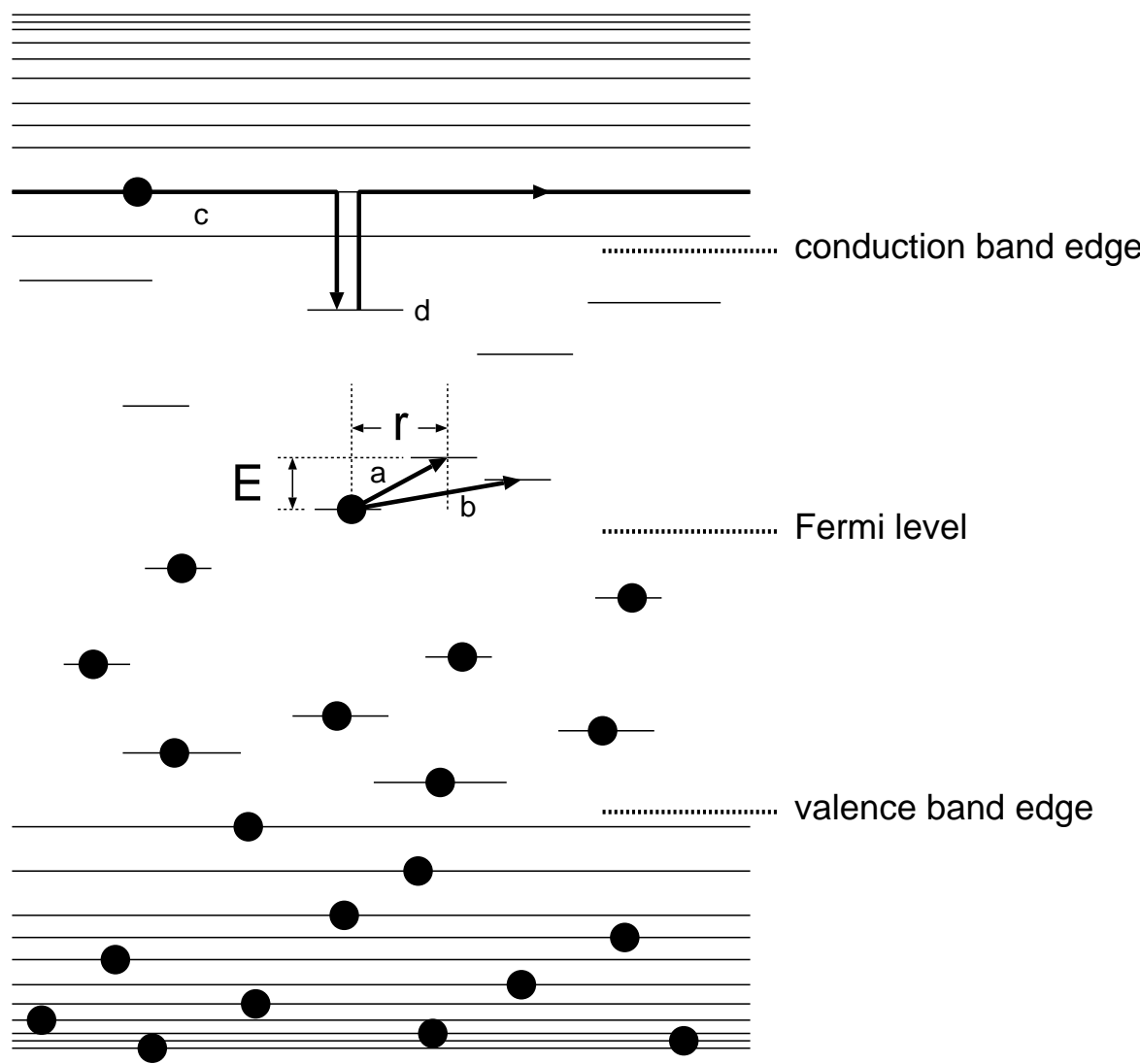

Figure 1.8: Distribution of states in an amorphous semiconductor (according to [Zal83]). Two basic classes of conduction mechanisms can be discerned, based either on tunneling (a,b) or thermal activation $(\mathrm{c}, \mathrm{d})$. 


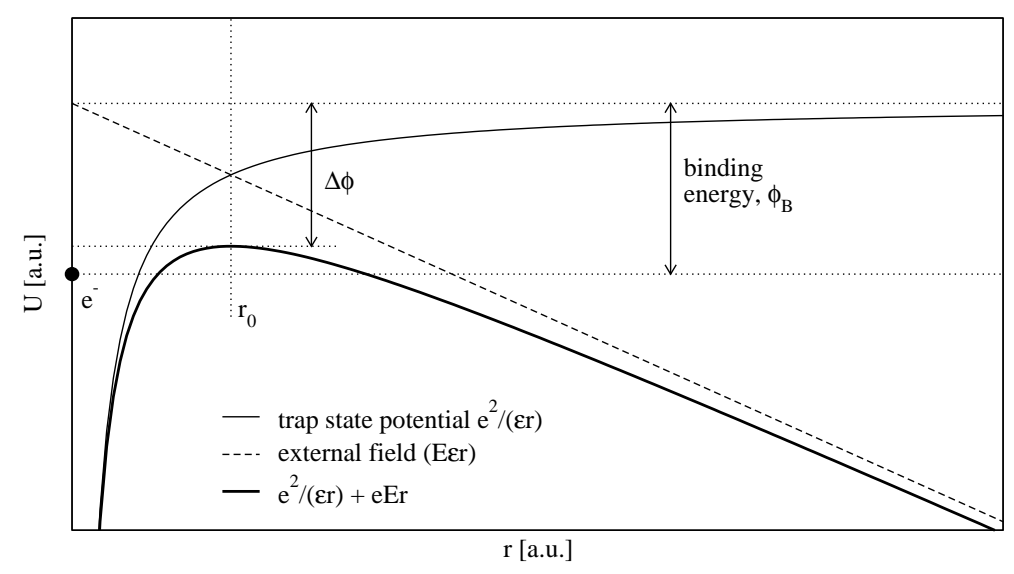

Figure 1.9: The coulomb potential $e^{2} / r \varepsilon$ of the ionized trap state is modified by an external field $E$, reducing the energy to free the electron by $\Delta \phi$. The resulting barrier $\phi_{B}-\Delta \phi$ can be overcome by thermal excitations.

\subsubsection{Frenkel-Poole conduction}

Frenkel-Poole conduction [Fre38] is a thermally activated hopping conduction in semiconductors with localized trap states. The charge carriers are caught in the potential wells of the trap states (fig. 1.9). The well depth $\Phi_{B}$, corresponding to the binding energy of the trapped electron, is assumed constant. Application of an external electrical field $E$ superposes a potential gradient $e E r$ over the well potential $e^{2} / r \varepsilon$. At $r_{0}$ with

$$
\begin{aligned}
\frac{d}{d r}\left[\frac{e^{2}}{\varepsilon r}+e E r\right]_{r_{0}} & =0 \\
\Rightarrow \quad r_{0} & =\sqrt{\frac{e}{\varepsilon E}},
\end{aligned}
$$

the potential barrier is lowered in the direction of the field by a an energy

$$
\Delta \Phi(E)=\beta_{F P} \cdot(\mathcal{E})^{\frac{1}{2}}
$$

with the Frenkel-Poole constant $\beta_{F P}=\left(e^{3} / \pi \varepsilon_{0} \varepsilon_{r}\right)$, and $\varepsilon_{0} \epsilon_{r}$ the high-frequency dielectric constant of the solid. At sufficiently high fields the barrier height $\Phi_{B}-$ $\Delta \Phi(E)$ trapping the electron can be low enough to allow the electron to escape 
the trap state through energy acquisition from random thermal excitations. This introduces a Bolzmann factor

$$
\exp \left(-\frac{\phi_{B}-\Delta \Phi(E)}{k_{B} T}\right)
$$

in the hopping rate, yielding the Frenkel-Poole equation

$$
I \propto E \cdot \exp \left(\frac{-q\left(\phi_{B}-\sqrt{q E / \pi \varepsilon_{0} \varepsilon_{r}}\right)}{k_{B} T}\right)
$$

This entirely one-dimensional treatment slightly overestimates the effective reduction of the potential barriers. The full barrier height reduction is only available exactly in the direction of the electrical field. Destination states however may be located off that direction.

The assumption of a constant trap state ionization energy does not apply to the amorphous carbon network, with a finite energy distribution in the localized band tail states. If the thermal energy is high compared to the barrier height distribution this effect can be neglected. At low temperatures, however, a significant deviation from the predicted current voltage curves is expected.

\subsubsection{Variable range hopping}

Variable range hopping is a tunneling process of charge carriers between localized states at low temperatures and low electrical fields. The destination state is chosen by two competing requirements. The expression for the tunneling probability through a potential barrier contains a factor

$$
\exp (-2 \alpha R)
$$

with $\alpha$ being the decay length of the wavefunction in the surrounding potential barrier. It has to be noted that the spatial extent of the localized state is not determined by $\alpha$, and usually is orders of magnitude larger. This factor makes tunneling to closest neighbors favorable. 
However, the electron has to acquire any difference in energy $\Delta E$ between the source and destination states through absorption of a phonon. The probability for an energy gain $\Delta E$ can be approximated by a Bolzmann distribution

$$
\exp \left(-\frac{\Delta E}{k_{B} T}\right)
$$

The availability of states within a small energy range $\Delta E$ is proportional to the considered hop-target volume $\frac{4 \pi / 3}{R}$. With a density of states $N(E)$ per unit energy and volume the closest available $\Delta E$ within a radius $R$ can be calculated as

$$
\Delta E=\left({\frac{4 \pi / 3^{3}}{R}}^{2} N(E)\right)^{-1} .
$$

Hence the further the electron can hop, the closer the source and destination states can match their energy, minimizing $\Delta E$. This introduces a competing factor which makes longer hopping distances favorable. The total probability for a certain hopping event is given by the product of the two factors above. Its minimum is located at

$$
R=\left(\frac{1}{8} \pi N(E) \alpha k_{B} T\right)^{\frac{1}{4}}
$$

yielding a conductance proportional to

$$
\sigma_{0} \cdot \exp \left(-\frac{B}{T^{1 / 4}}\right)
$$

with

$$
B=2\left(\frac{3}{2 \pi}\right)^{\frac{1}{4}}\left(\frac{\alpha^{3}}{k_{B} N(E)}\right)^{\frac{1}{4}}
$$

This simplified treatment, however, does not account for either population probabilities of source or destination states, local chemical potentials or spatial correlations. At moderate electrical fields a percolation based [Pol76] approach can be taken, extending eq. 1.11 to

$$
I \propto \exp \left(-0.9 \cdot \frac{B}{T^{1 / 4}}+\frac{0.17 R \cdot e E}{k_{B} T}\right)
$$


with the characteristic hopping distance $R$. The validity of the expansions and approximations used is restricted to the low-field regime, defined by $e E a / 2 k_{B} T<1$, with $a$ being the Bohr radius of the localized states.

In high electric fields, a hopping electron can acquire a considerable potential energy relative to the Fermi level of the sample. If the energy gain cannot be fully compensated through phonon emission a temperature increase of the current carrying electrons will happen, creating a non-equilibrium energy distribution. Depending on the acquired electron temperature an activation-less conduction state with virtually free carriers can be reached, increasing the sample conductivity.

\subsubsection{Current distribution}

Conductivity measurements of single ion tracks with the AFM require a thorough investigation of current path within the sample. A point-like contact can be realized on the top surface of the sample using an electrically conductive cantilever. The back contact is given by the silicon substrate, connected over the entire area of the film, yielding an asymmetric electrode setup.

The sample was approximated by an ohmic resistor network. For calculation purposes only a single ion track embedded in a ta-C matrix was considered. In this cylinder-symmetrical setup no angular contributions of current and electrical field are expected, thus every node at a certain radius and height in the film lies on equal potentials and has an equal current flow in axial and radial directions.

Potentials and currents in a resistor network obey Kirchhoff's circuit laws. The whole problem can be formulated as a linear equation system and its solution leads to the potentials at the nodes. This formalism is described in detail in appendix A. The top viewgraph in fig. 1.10 shows the computed current distribution for an unirradiated matrix with a conductivity $\sigma_{0}$. The current path widens toward the bottom, yielding an increased current carrying volume compared to a straight path from the top contact to the bottom contact. The bottom viewgraph shows the current path in the same matrix after a track with a conductivity of $10^{3} \cdot \sigma_{0}$ is introduced. The charge carriers, taking the path of least resistance, are confined mostly to the track. Although the track constitutes a local conductivity increase by three orders of magnitude, the calculated current increases only by a factor of 

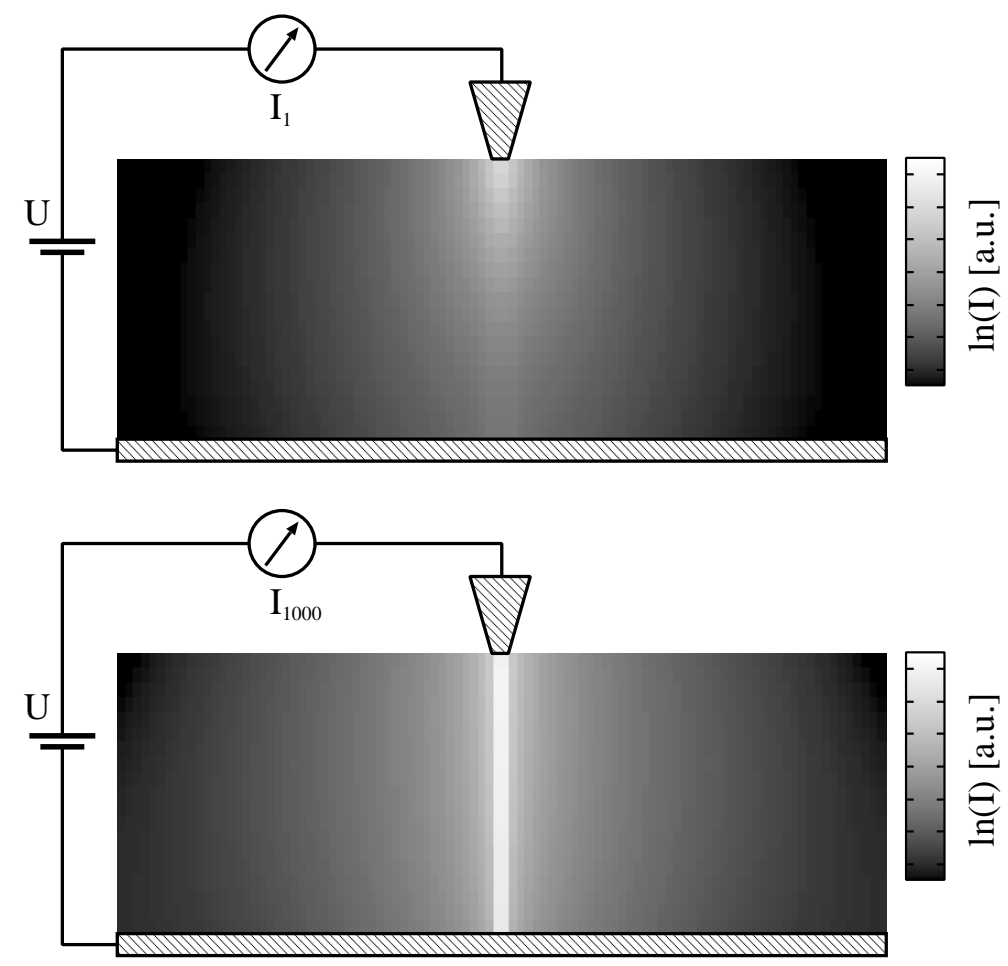

Figure 1.10: Top: computed current distribution for an unirradiated matrix with a conductivity $\sigma_{0}$. The current path widens toward the bottom, yielding an increased current carrying volume compared to a straight path from the top contact to the bottom contact. Bottom: current path in the same matrix after a track with a conductivity of $10^{3} \cdot \sigma_{0}$ is introduced. The charge carriers, taking the path of least resistance, are confined mostly to the track. 


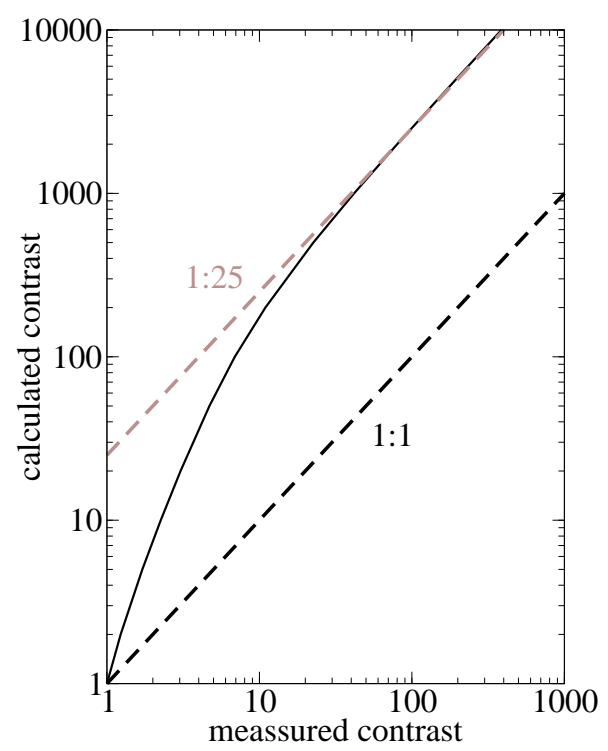

Figure 1.11: The relation of effectively measured conductivity contrast to actual (calculated) conductivity contrast. The measurement underestimates the contrast of track and matrix by about an order of magnitude, due to a change in current carrying volumes. The ratio 1:1, corresponding to the identity of measured and calculated effective contrast, and the asymptotic 1:25 ratio of the measured and calculated contrast are shown as dashed lines. For high currents the measurement underestimates the conductivity contrast by a factor of 25 . 
50. This results in an underestimation of the specific conductivity of the track material from the AFM measurements by about a factor of 20. This factor model is independent of the absolute scales of voltage and conductivity, and only depends on the aspect ratio of the track. Figure 1.11 shows the asymptotic behaviour of the factor for an aspect ratio of 1:20. For that reason the huge difference in current carrying volume must be accounted for when calculating the specific resistivities from AFM current measurements. 


\section{Chapter 2}

\section{Computational methods and results}

\subsection{Molecular dynamics simulations}

A molecular dynamics simulation numerically computes the phase space trajectories of an ensemble of particles governed by a programmed set of interaction laws or inter-particle potentials.

There are many existing studies on atomistic MD simulations of ion and cluster bombardment of diamond and graphite at energies from eV to keV [Smi90, Smi96, Ker99, Nor96, Sal01, Mar06, Saa99, Mar02, Mar97] using classical empirical potentials like the Tersoff [Ter88], environment-dependent interatomic potential (EDIP), [Mar97],reactive empirical bond order (REBO) potential [Bre90], adaptive intermolecular reactive empirical bond order (AIREBO) potential [Stu00], and tight-binding simulations [Saa99, Mar02, Mar97]. Previous simulations typically involved $10^{3}-10^{5}$ atoms and focused on the production of point defects [Smi90, Smi96, Nor96, Saa99, Mar02, Mar97] or sputtering [Ker99, Sal01, Mar06]. On the other hand, there seems to be only one attempt to model MeV-GeV bombardment: heating of small carbon volumes, surrounded by a few layers of cold material to mimic fast ion tracks, was simulated using tight binding for up to 432 atoms [Sor04]. It was found that the $\mathrm{sp}^{2} / \mathrm{sp}^{3}$ bonds ratio depended on the track temperature and the relative amount of hot and cold material. In order to study the cooling of a hot track due to heat, mass and momentum transport, much larger samples are needed. This track evolution has been studied with many semi-analytical models for tracks in semiconductors and insulators: the Coulomb 
explosion model [Fle75], several flavors of the thermal spike model [Vin76, Joh80, Sig81, Sze97, Wan94, Tou02, Vol02], etc. The thermal spike model assumes that the temperature profile in the hot track evolves according to a heat diffusion equation. This temperature history can be used to calculate defect production [Vin76], amorphization [Sze97, Wan94, Tou02], sputtering [Joh80, Sig81, Tou02], etc. MD simulations of thermal spikes [Urb94, Bri99, Jak02, Beu03, Tuc05, Bri02a], using nonreactive pair potentials and $10^{4}-10^{6}$ atoms, have shown that these semi-analytical approaches are valid only when the kinetic energy corresponding to the initial temperature of the track is much smaller than the binding energy of the material. Otherwise, there is not only heat transport, but also pressure and mass transport [Urb94, Bri99, Jak02], and the semi-analytic assumptions break down. MD simulations comparing Coulomb explosion models to thermal spike models found no intrinsic difference between the two models [Bri02a] in the sense that a hot track will evolve in roughly the same fashion independently of how the heat was delivered to the track atoms (by Coulomb explosion, secondary electron heating, etc.). Thanks to advances in computer power, large-scale thermal spike simulations with more than a million atoms can now be carried out in complex materials like silicates and water ice.

A series of MD simulations was performed to gain insight into the track formation process. The temperature evolution in and around the thermal spike and material transport were of special interest. The latter includes radial transport and density profiles as well as parallel transport along the track and surface effects such as sputtering and hillock formation.

\subsubsection{Interatomic potentials}

Numerical accuracy and physical validity of the particle interaction model depends on numerical accuracy of the integration and physical accuracy of the interaction potential. Atomic bonds are formed by electron orbitals which call for a quantum mechanical treatment. However, the numerical solution of the Schrödinger equation is a computationally extremely expensive task and cannot be applied to study the dynamics of large $(>10000)$ particle ensembles even after approximations such as density functional theory (DFT) [Hoh64, Koh65] and Car-Parinello MD [Car85]. 
Instead, a phenomenological approach can be taken, setting aside the dynamics of the electronic system. The atomic interactions are described by an analytical potential as a function of the atom coordinates. Careful observation of the different bonding states in a system can guide the construction of such a potential function and its constituent terms. Such are two- and three-body interactions, and bond-order terms. The free parameters of this empirical potential can be fitted to experimental data from bulk samples and DFT simulations on small samples.

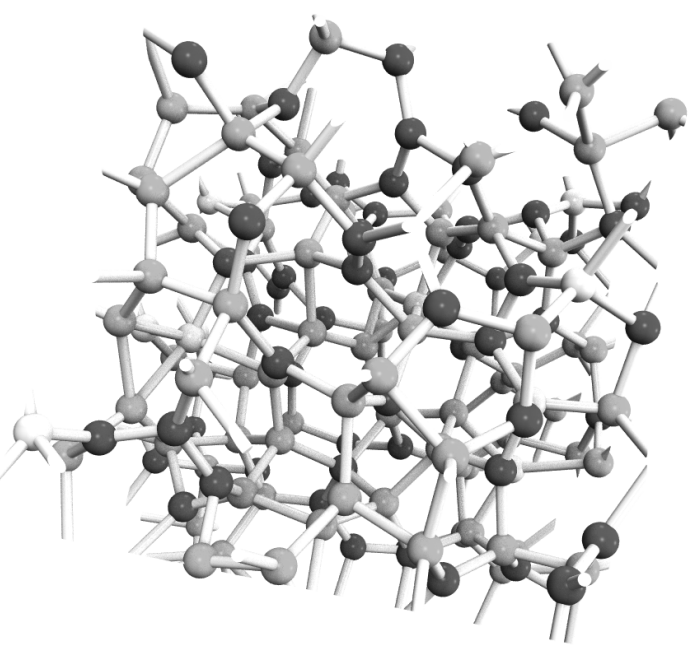

Figure 2.1: 3 D rendering of an amorphous carbon network created with a liquid quench technique. Three- (black) and fourfold (grey) coordinated atoms meshed together . Their ratio determines to properties of the material. This sample shows a few overcoordinated atoms (white).
For the simulations presented here the first parametrization of the REBO potential by Brenner [Bre90] was used. Numerical integration was performed with the MD software package MDCASK [Rub90] with minor modifications applied. The REBO potential has a possible problem: when a melt is rapidly quenched it produces a graphitic-rich structure even at densities close to the diamond density, which is in disagreement with experimental results [Mar02, Mar97, Jäg00, Stu06]. This might affect the late evolution of our track, but the influence in the amorphization threshold would be small, given that melting is reasonably described by the Brenner potential [Glo99]. The potential by Tersoff [Ter88] has a similar problem [Mar02], while EDIP [Mar02, Mar97], REAX potentials, and tight binding simulations [Smi96, Sor04] produce reasonable results. Source of the problem with the REBO potential is the low cut-off distance of $2 \AA$, which assumes that above this cut-off there is no further interaction between atoms. This cut-off is appropriate for diamond and for the in-plane graphite interactions, but it is shorter than the separation of $3.35 \AA$ between graphitic sheets. Thus the potential allows for an unphysical graphite-like structure with an interplane spacing of $2 \AA$ yielding a density about $7 \%$ higher than diamond. Jäger and Albe proposed [Jäg00] a 

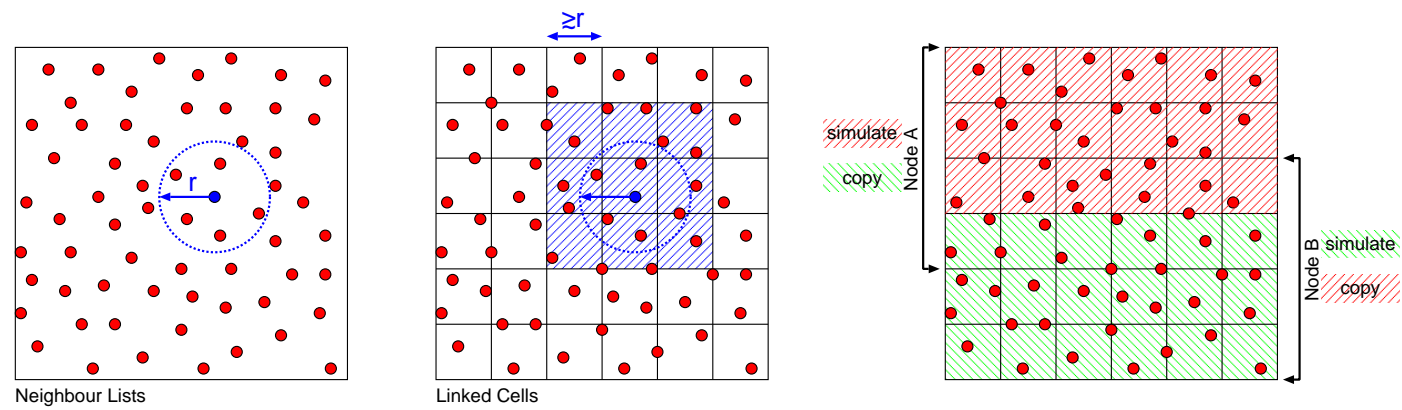

Figure 2.2: Schematic illustration of the optimization steps needed to reduce the complexity of a REBO simulation from $O\left(N^{3}\right)$ to $O(N)$ and allow for parallelization. Left: the introduction of neighbor lists, Center: . Right: each block of link cells is treated on a different computation node, the nodes only have to exchange information about the state of the so called skin cells, bordering cells in adjacent blocks.

slight increase of the REBO potential cut-off distance by $12.5 \%$. We implemented this change in the MDCASK software and subsequent simulations confirmed an increase of the $\mathrm{sp}^{3}$ content in the amorphous samples, enabling us to reproduce the hybridization ratio of ion-beam deposited thin film samples (fig. 2.1). The $\pi$-bond interaction in graphite can be modeled by adding a van der Waals interaction using a Lennard-Jones contribution to the total energy [Smi96, Ker99, Stu00]. However, the drawback of this more accurate solution is a massive increase in interaction distance which affects the computational cost with its third power. MD simulations of radiation damage in graphite by $\mathrm{eV}-\mathrm{keV}$ ion and cluster bombardment seem to indicate that the effect of the long range term in the final defect production is small [Smi96, Ker99] and, therefore, it has been neglected in our simulations. At small interatomic distances, the REBO potential was splined to the universal Ziegler-Biersack-Littmark (ZBL) potential [Zie85] to describe energetic collisions.

\subsubsection{Implementation details}

The REBO potential contains three-body interaction terms which require an iteration over all atom triplets in the sample. This sets the complexity of the unoptimized computation to $O\left(N^{3}\right)$ and severely limits the simulation sample size. The finite range $r_{\text {cut }}$ of the potential allows to discard all triplets in which at least one interatomic distance exceeds $r_{\text {cut }}$. By introducing a list for each atom, which contains 
all neighbors within a fixed range $r_{N}$, the triplets can be chosen from connected sets of atoms. A set of atoms shall be referred to as connected whenever each atom is contained in the neighbor list of at least one other atom in the set. Neighbor list creation involves iterating over all atom pairs, selecting only those pairs which have a distance of less then $r_{N}$. Iteration over pairs has a computational complexity of $O\left(N^{2}\right)$, which, however, can be reduced by spatially sorting the atoms in the sample. The sample volume is divided into a three-dimensional array of cells and a list is linked to each box enumerating all atoms contained in the box. In doing so, the neighbor list creation can be be restricted to the pairs of atoms that fall in the same cell or in adjecent link cells; this spatially sorted truncation allows for a reduction of the computational complexity to linear scaling $O(N)$.

To parallelize the problem and spread it over several computation nodes, the array of link cells is subdivided into blocks. Each block of cells is then treated on a different node. The nodes only have to exchange information about the state of the so-called skin cells, bordering cells in adjacent blocks.

The accuracy of the numerical integration depends on the time step size. Between two simulation steps no atom should move further than a fixed distance $\Delta r_{\max }$, chosen in such a way that the potential can be assumed linear. For each simulation step the time step can be computed from the maximum atom velocity in the previous step. Caveat is the dependence of the time step on the maximum velocity of potentially very few atoms. As the atom velocity at constant temperature inversely depends on the atom masses, the use of very light atoms such as hydrogen is severely penalized in terms of computational expense. Therefore in this study the ion irradiation of hydrogenated amorphous carbon (a-C:H), prevalent in plasma deposited carbon films, had to be excluded.

\subsubsection{Sample preparation}

The diamond and graphite samples were prepared as ideal crystals by replication of their respective unit cells. The pristine ta-C samples were created using a rapid quench method. Starting point was diamond crystal with an adjusted lattice constant of $3.7 \AA$ and a size of $10 \times 10 \times 10$ lattice units. Full periodic boundary conditions were applied, the crystal was heated to $10000 \mathrm{~K}$, held at that temper- 
ature for $0.5 \mathrm{ps}$, and then quenched stepwise by velocity rescaling to successively $7000 \mathrm{~K}, 5000 \mathrm{~K}$, and finally $300 \mathrm{~K}$. At each temperature step the material was relaxed for about $10 \mathrm{fs}$, after the final temperature step another $0.1 \mathrm{ps}$ of relaxation with temperature rescaling to $300 \mathrm{~K}$ every five MD steps were added to minimize the potential energy of the sample. The initial choice of a $3.7 \AA$ lattice constant results in a material with a density of $3.15 \mathrm{~g} / \mathrm{cm}^{3}$, which corresponds to the density of the films used in the irradiation experiments. This $(3.7 \mathrm{~nm})^{3}$ ta-C building block was then used to create arbitrarily-sized ta-C samples by periodic replication.

A coordination analysis of the material by neighbor counting shows a $62 \% \mathrm{sp}^{3}$ and $37 \% \mathrm{sp}^{2}$ content, with the remainder being either over or under coordinated. Since the stress

$$
\sigma=-\frac{\partial U}{\partial V}
$$

is the partial derivative of the free energy $U$ with respect to the volume $V$, a stress analysis boils down to a series of free energy calculations with the position vectors of the sample rescaled by a factor of $(1 \pm \epsilon)$.

Figure 2.3 shows the results for the ta-C building block (top) in comparison to a diamond sample (bottom). The hollow squares show the free energy curve for the ta-C sample calculated using the original REBO parameterization from [Bre90], while the filled squares were calculated using the modified [Jäg00] REBO potential. The ta-C sample in the modified REBO potential shows a compressive stress of about $7 \mathrm{GPa}$, which is in good agreement with experimental data [McK91, Fal93, Ron00]. For diamond the two parameterizations yield exactly the same values, since the correction only affects interatomic distances above $1.7 \AA$. The diamond bulkmodulus, calculated from the parabolical fit, is $255 \mathrm{GPa}$, which is slightly below the literature value of about $440 \mathrm{GPa}$ [McS57]. The amorphous carbon sample in the modified Brenner potential yields a bulkmodulus of $570 \mathrm{GPa}$.

For the bulk simulation runs samples with sizes ranging from $21 \times 21 \times 7 \mathrm{~nm}^{3}$, to $43 \times 43 \times 7 \mathrm{~nm}^{3}$ for diamond $\left(5.76 \cdot 10^{5}-2.3 \cdot 10^{6}\right.$ atoms), $21 \times 21 \times 7 \mathrm{~nm}^{3}$ for graphite $\left(3.83 \cdot 10^{5}\right.$ atoms $)$ and amorphous carbon $\left(5.76 \cdot 10^{5}\right.$ atoms $)$ were prepared. The largest runs were comprised of a series of ta-C samples with a free surface and a volume of $48 \times 48 \times 14 \mathrm{~nm}^{3}$ containing $5.0 \cdot 10^{6}$ atoms, requiring a total of about 140000 CPU-hours. The simulation volume cross sections were chosen to be much 


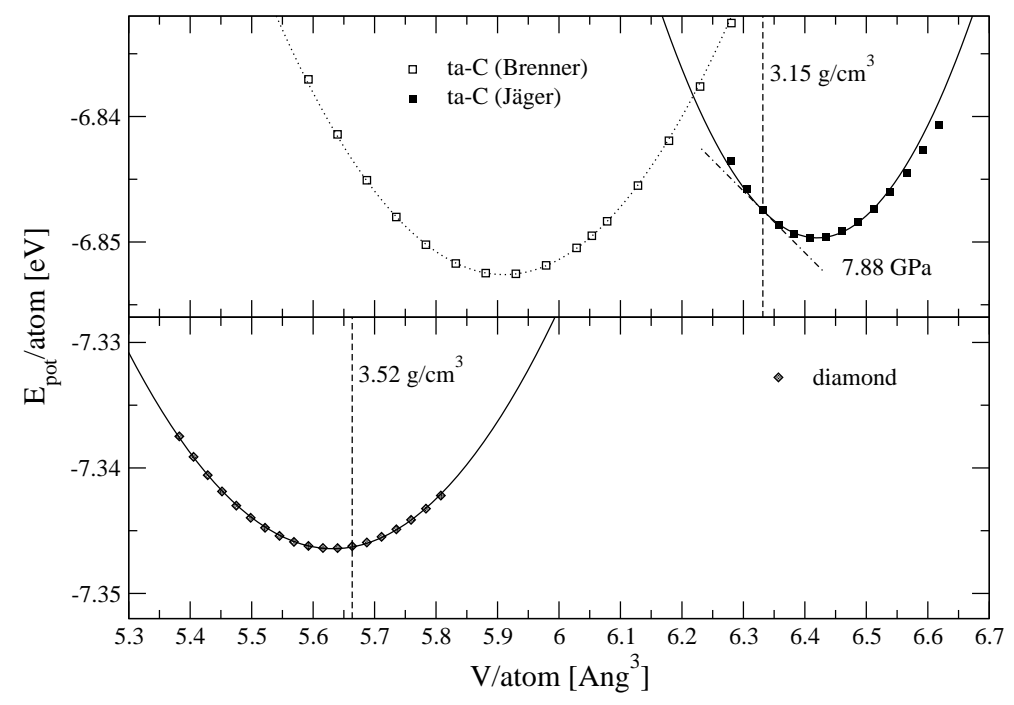

Figure 2.3: Potential energy over volume per atom for diamond and ta- $C$ in the original Brenner parametrization [Bre90] and with the corrections proposed by Jäger and Albe [Jäg00]. The two parametrizations yield exactly the same values in the case of diamond, since the correction only affects interatomic distances above $1.7 \AA$. The ta-C sample, generated by liquid quenching shows a high compressive stress of $7.88 \mathrm{GPa}$, which is in good agreement with experimental data [McK91, Fal93, Ron00]. All simulations below were performed with the corrected potential. 
larger than the initial excited track size. With these sample sizes we can include not only the hot track, but a large amount of pristine, cold material outside the track. The interface between the hot track and the cold sample can be tracked with time, as in the thermal spike model used by Toulemonde and co-workers to calculate track sizes [Wan94, Tou02].

\subsubsection{Simulation setup}

None of the available empirical potentials include any form of electronic excitation. Thus, the interaction and predominately electronic energy loss processes of the passing swift heavy ion have to be dealt with separately. High projectile velocities of the order of $0.05 \cdot c$ vindicate the treatment of the initial energy deposition process as an instantaneous event occurring at time $t_{0}^{e}$. The spatial extent of the electronic excitations along the track at $t_{0}^{e}$ is approximated by the Bohr adiabatic radius (eq. $1.1)$.

We assume that the timescales for the diffusion of electronic excitation $E^{e}$ and the conversion of electronic to lattice excitation $E^{T}$ are much shorter than the diffusion of lattice heat. With the approximation that the transformation of the former to the latter is fully concluded before the aforementioned conversion, the electronic excitations in the swift ion tracks can be simulated as prompt cylindrical spikes, as it has been done before for other materials using pair potentials [Urb94, Bri99, Jak02, Beu03, Bri02a] and embedded-atom method potentials [Tuc05].

We use an initial track radius within which atoms are energized by electronic excitations. This track radius, $R_{\text {track }}(t=0)$, can be taken as the Bohr track radius [Joh93b], or as some function of the ion energy, velocity, etc. [Wan94, Tom94]. We choose an initial track radius of $3 \mathrm{~nm}$, which is similar to the track radius used to study cratering and damage in hydrocarbons [Bri02b]. Recent measurements of track radii in amorphous carbon give a final track radius of less than $5 \mathrm{~nm}$ for bombardment with $350 \mathrm{MeV}$ gold (Au) ions [Sch04].

Since we are interested in bulk effects, periodic boundary conditions are used in all directions (we are simulating an infinite track). The track axis was set along the [001] direction and in order to avoid artifacts due to size-effects, the height of the track is at least two times the track radius. 


\begin{tabular}{l|c|c|c|c|c|c|c|c} 
& $10 \%$ & $20 \%$ & $30 \%$ & $40 \%$ & $50 \%$ & $70 \%$ & $90 \%$ & $100 \%$ \\
\hline Graphite & 0.9 & 1.8 & 2.7 & 3.5 & 4.4 & 6.3 & 8.0 & 8.9 \\
\hline Diamond & 1.5 & 3.0 & 4.5 & 6.0 & 7.5 & 10.5 & 13.5 & 15.0 \\
\hline ta-C & 1.3 & 2.7 & 4.0 & 5.4 & 6.7 & 9.4 & 12.0 & 13.4
\end{tabular}

Table 2.1: Effective energy losses in $\mathrm{keV} / \mathrm{nm}$ for graphite, diamond, and ta-C simulation runs.

A fraction of atoms within $R_{\text {track }}(t=0)$ are each given an excitation energy $\mathrm{E}_{\text {exc }}$, which determines an effective stopping power $d E / d x$. The exact value of $\mathrm{E}_{\text {exc }}$ received by the atoms due to decay of the electronic excitations is not known. Abinitio tight-binding simulations of laser-induced electronic excitation in graphite and diamond have considered temperatures higher than $3 \mathrm{eV} /$ atom [Jes01]. In addition, experimental measurements of the electronic temperature in ion-irradiated amorphous carbon using Auger electrons [Sch99] give an upper limit to the temperature in diamond and graphite tracks. These measurements indicate temperatures of several tens of thousands of $\mathrm{K}$ after $\approx 10 \mathrm{fs}$. Excitation energy was chosen to be $3 \mathrm{eV} /$ atom, giving an initial temperature of $\approx 23000 \mathrm{~K}$ for the fully excited track. This energy is comparable to the one used in [Sor04], where the upper limit studied for the track temperature was $30000 \mathrm{~K}$. A variable time step algorithm [Rub90] is employed in our simulations, so that time steps much smaller than $1 \mathrm{fs}$ are used in the early phase of the spike, while time steps of $\approx 1 \mathrm{fs}$ can achieve energy conservation at longer times when the spike is cooler. Energy can be given to excited atoms as a delta function or as a Maxwellian distribution with mean energy $E_{\text {exc }}$. These two excitation modes only differ when $E_{\text {exc }} \ll U$, [Bri99] where $U$ is the binding energy of the material, which is $\approx 7 \mathrm{eV}$ for diamond and graphite. Here we use a delta function distribution, since we are exploring the regime where $E_{\text {exc }} \approx \frac{U}{2}$. We assume that all excitations decay at the same time. This approximation can be relaxed, leading only to relatively small corrections [Beu03].

Given that diamond is a semiconductor and that graphite is a 2D conductor (along the graphene sheets), it could be expected that the initial electronic excitations will be rapidly quenched or diffuse away from each other. This means that, even if the entire volume of the track was initially excited, only a fraction of these excitations will decay close to each other and to the center of the track. Only a 
high concentration of excitations will lead to significant damage production [Jak02]. Excitations decaying far from the track have small probability of producing lasting damage, since the individual excitation energy is small compared to the binding energy of the material and the displacement energy. In particular, the displacement energy in diamond is larger than $15 \mathrm{eV}$ [Smi90, Smi96], so a single excitation of $3 \mathrm{eV}$ will only lead to local heating without any defect being created in the lattice. The quenching and migration of excitations is taken into account using a simple model: for the same track radius and excitation energy $E_{\text {exc }} /$ atom, the role of the efficiency of the electron-phonon coupling is examined by assuming different numbers of excited atoms, given as a fraction of the total number of atoms in the initial track. Efficiencies of 10, 20, 30, 40, 50, 70, 90 and 100\% are considered. The tracks had a total of 35587, 23651, and 33033 atoms for diamond, graphite, and amorphous carbon respectively. Therefore, these values would correspond to effective $d E / d x$ values of $1.5-15 \mathrm{keV} / \mathrm{nm}$ in diamond, $0.9-8.9 \mathrm{keV} / \mathrm{nm}$ in graphite, and $1.3-13.4 \mathrm{keV} / \mathrm{nm}$ in ta-C (see table 2.1 ). $100 \%$ efficiency would correspond to an energy of 47/43/50 MeV for Au bombardment of diamond/graphite/ta-C.

As a summary, this model could be used to compare to ion bombardment experiments for a given ion-target pair (for a given ion mass, velocity, and charge, etc.) using two free parameters: a) the track radius; b) the efficiency of the transfer of electronic excitations to the lattice that links the effective $d E / d x$ with the experimental $d E / d x$.

\subsubsection{Results}

\section{Bulk effects $42 \mathrm{~nm}$}

Fig. 2.5 shows four frames of the diamond simulation runs at $40 \%(6.0 \mathrm{keV} / \mathrm{nm})$,

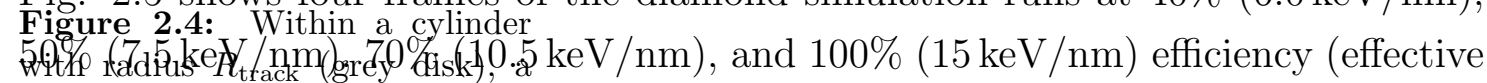

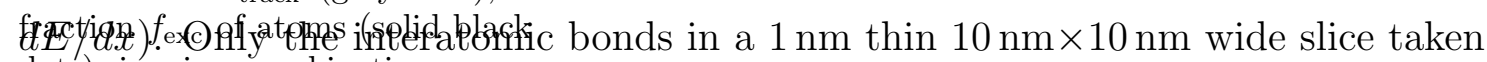
dots) is given a kinetic energy Eexpendicular to the track are shown. All runs below $50 \%$ efficiency show only isolated point defects. At 50\% efficiency the center of the track region is amorphized, while the periphery stays crystalline, indicating that heat dissipation competes with 


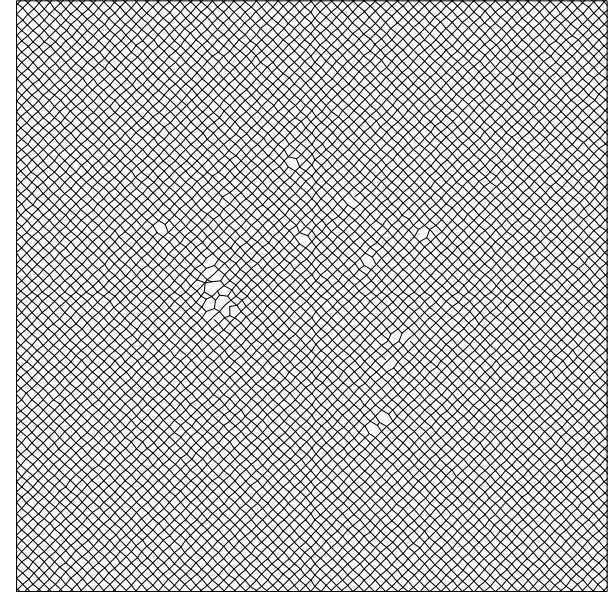

$40 \%$

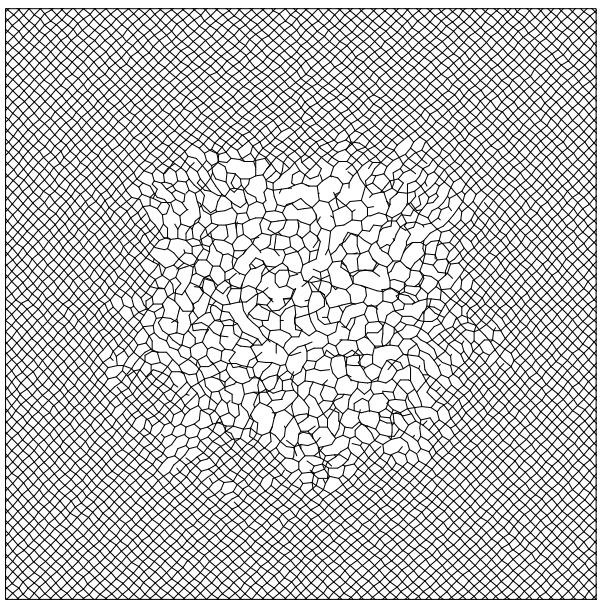

$70 \%$

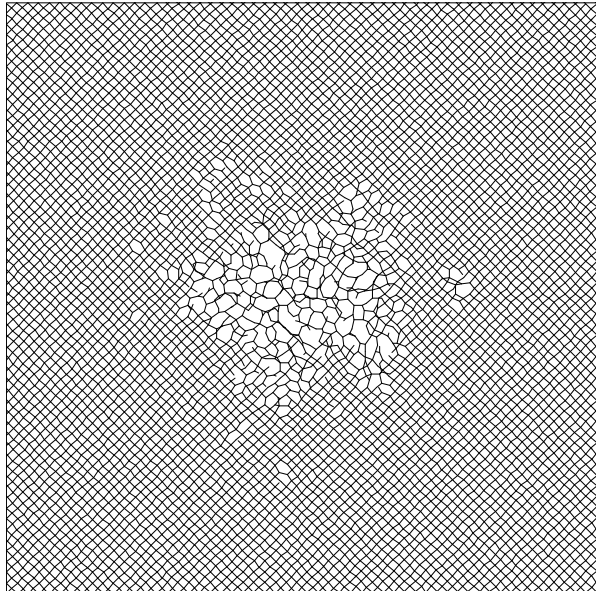

$50 \%$

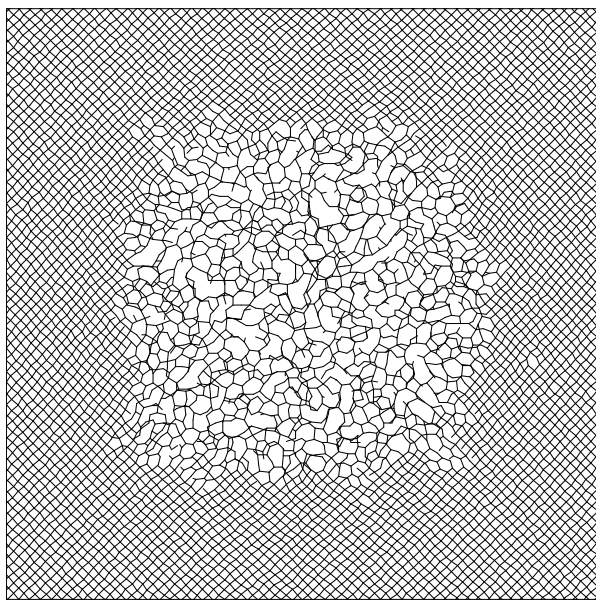

$100 \%$

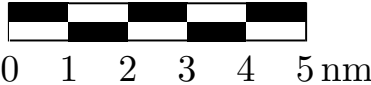

Figure 2.5: Four frames of the diamond simulation runs at $40 \%(6.0 \mathrm{keV} / \mathrm{nm}), 50 \%(7.5 \mathrm{keV} / \mathrm{nm})$, $70 \%(10.5 \mathrm{keV} / \mathrm{nm})$, and $100 \%(15 \mathrm{keV} / \mathrm{nm})$ efficiency (effective $d E / d x)$. Only the interatomic bonds in a $1 \mathrm{~nm}$ thin $10 \mathrm{~nm} \times 10 \mathrm{~nm}$ wide slice taken perpendicular to the track are shown. Runs below $50 \%$ efficiency show only isolated point defects. 


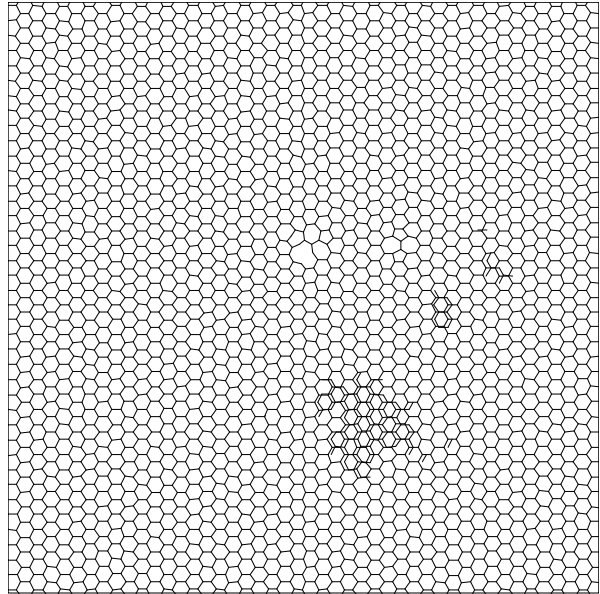

\section{$40 \%$}

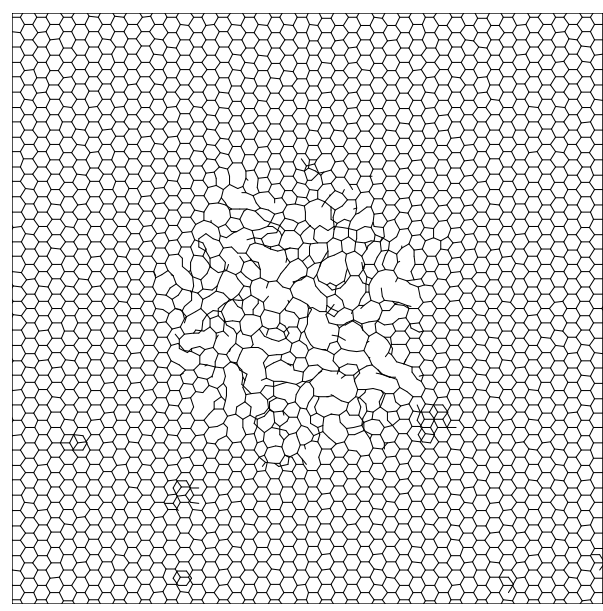

$70 \%$

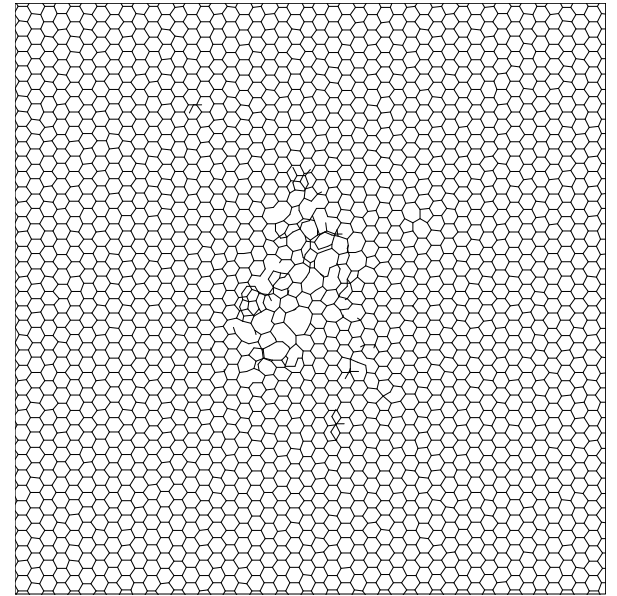

$50 \%$

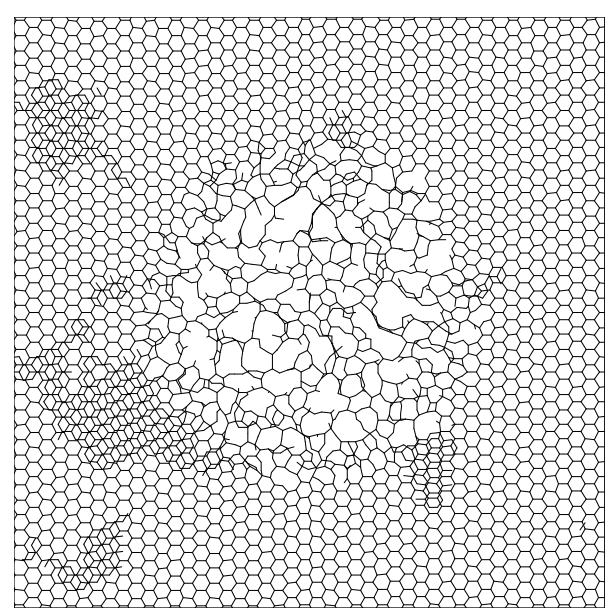

$100 \%$

$\begin{array}{llllll}0 & 1 & 2 & 3 & 4 & 5\end{array}$

Figure 2.6: Four frames of the graphite simulation runs at $40 \%(3.5 \mathrm{keV} / \mathrm{nm}), 50 \%(4.4 \mathrm{keV} / \mathrm{nm})$, $70 \%(6.2 \mathrm{keV} / \mathrm{nm})$, and $100 \%(8.9 \mathrm{keV} / \mathrm{nm})$ efficiency (effective $d E / d x)$. Only the interatomic bonds in a $1 \mathrm{~nm}$ thin $10 \mathrm{~nm} \times 10 \mathrm{~nm}$ wide slice taken perpendicular to the track are shown. Runs below $50 \%$ efficiency show only isolated point defects. Due to the missing interplane forces the parts of neighboring graphene sheets bend into the depicted volume slab creating extra graphene patches. 
track formation. The same series for graphite is depicted in fig. 2.6. Again the amorphization threshold lies at 50\% efficiency, corresponding to $4.4 \mathrm{keV} / \mathrm{nm}$ in graphite.

At the start of the simulation the graphene sheets lie in $\mathrm{x}-\mathrm{y}$ planes perpendicular to the track. However, as a result of the missing interplane forces in the REBO potential the graphene sheets can relax in-plane biaxial compressive stress by bending in z-direction. This is visible in fig. 2.6 as graphene patches are bending into the depicted volume slab.

The radial distribution function (RDF) $g(r)$ can be taken as a measure for close and medium range ordering in an atomistic sample. $g(r)$ is calculated as the mean particle density in all spherical shells of radius $r$ centered on a particle in the sample. In essence the RDF is a histogram of all interparticle distances normalized by volume. For an ideal crystal the RDF shows sharp lines, as the sample contains only a few classes of atoms with each member having an identical neighborhood. In the case of graphite and diamond, every atom has the exact same neighbor distribution. Samples at finite temperatures show peak broadening due to lattice vibrations. Amorphous samples show wide peaks for their first neighbors, centered around the preferred bond length. Second and third neighbor peaks rapidly decrease, approaching a constant distribution due to lack of medium and long range order.

Figure 2.7 shows the distribution functions for selected efficiencies in diamond, graphite, and amorphous carbon. Both diamond and carbon show less pronounced peaks at $50 \%$ efficiency than at $40 \%$, indicating a beginning amorphization. At $70 \%$ efficiency and above, both materials exhibit no peaks outside the second neighbor shell, indicating complete amorphization. In the amorphous carbon sample little or no medium range structure can be observed. Increasing efficiencies lead to a gradual change in first to second neighbor peak height ratio and their widths. This can be explained by a density decrease in the track which adds more freedom for a random distribution. At densities close to that of diamond, the denser $\mathrm{sp}^{3}$ hybridization is preferred over the $\mathrm{sp}^{2}$ which leads to a low density material. At lower densities this preference disappears and more $\mathrm{sp}^{2}$ hybridized sites are permitted. The amorphous sample shows a small spike at $2.25 \AA$, which coincides with the modified REBO cut-off. This indicates the need for further relaxation after the quenching during 


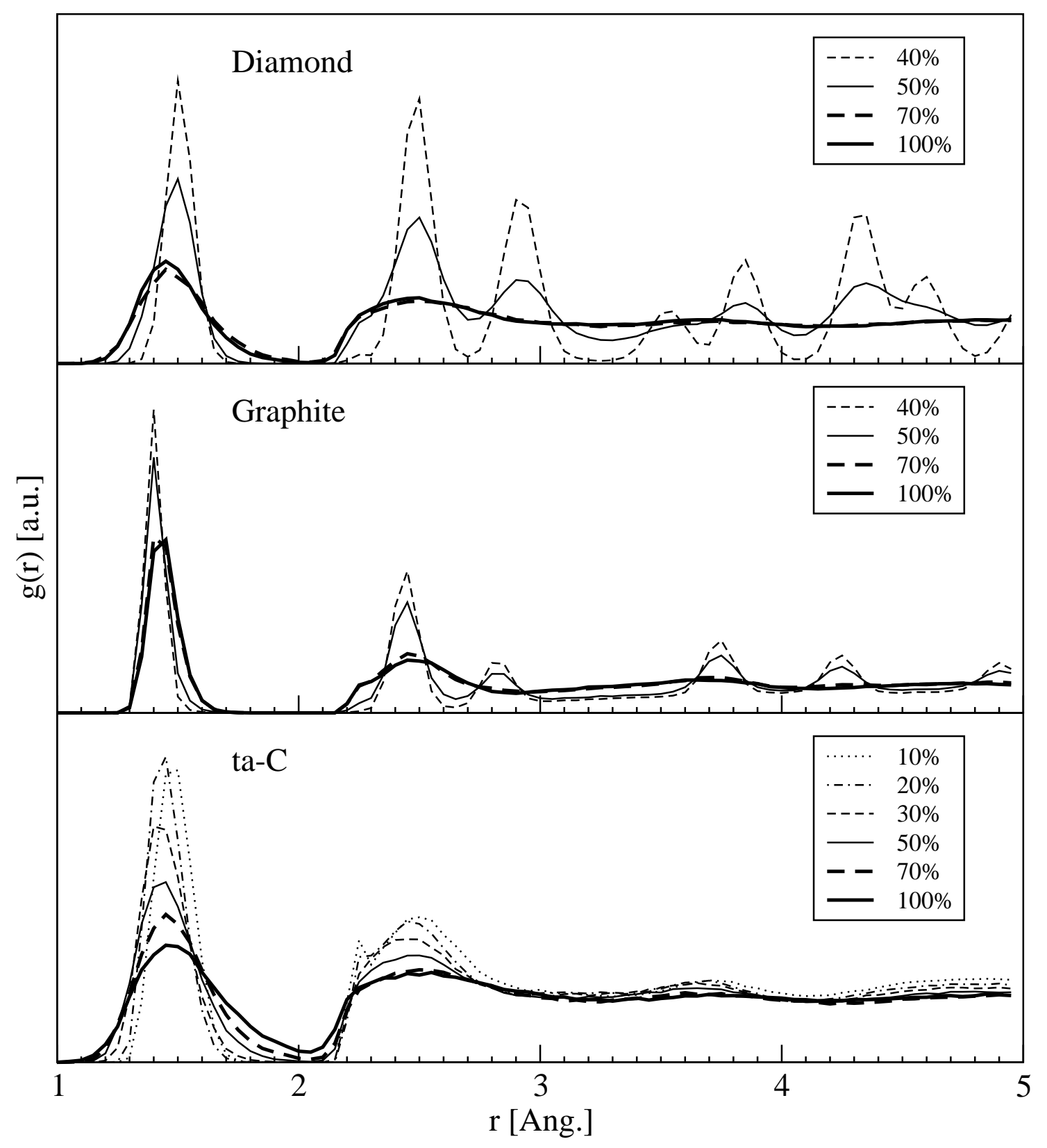

Figure 2.7: Radial distribution functions calculated from a cylindrical volume with 1 nm radius in the track center for selected efficiencies in diamond, graphite, and amorphous carbon. Both diamond and carbon show less pronounced peaks at $50 \%$ efficiency than at $40 \%$, indicating a beginning amorphization. At $70 \%$ efficiency and above both materials exhibit no peaks outside the second neighbor shell, indicating complete amorphization. The fully amorphized states for all materials show different first neighbor peak widths and heights, as would be expected from amorphous materials with different densities. 
sample preparation. However, the computational costs of longer relaxation times exceed the scope of this work.

Both diamond and graphite exhibit an amorphization threshold in the simulations. Different criteria for track formation have to be established for the already amorphous ta-C samples. In experimental observations the tracks can be detected through electrical conductivity contrast in the AFM (see chapter 3.1.1. A conductivity increase implies a change of the local bond structure from predominately $\mathrm{sp}^{3}$ to $\mathrm{sp}^{2}$. As the electronic system is not explicitly part of the simulation, the hybridization has to be inferred from the coordination number. Every fourfold coordinated atom is taken to be in a $\mathrm{sp}^{3}$ hybridiation state and every threefold coordinated atom is taken to be in a $\mathrm{sp}^{2}$ state. Any atom within a $1.9 \AA$ radius is considered a neighbor. This distance is derived from the first minimum of the radial distribution function in amorphous carbon. The angular distribution of the neighbor atoms is disregarded as the correlation of angles and coordination is to be taken care of by the REBO potential function. Any deviation from ideal angles is regarded as bond strain.

Radial averages of atomic coordination numbers have been calculated for all samples and are shown in fig. 2.8. Both diamond and graphite show pronounced differences between the $40 \%$ and $70 \%$ efficiency runs, with an intermediate step at $50 \%$. In diamond the track manifests as a region of decreased $\mathrm{sp}^{3}$ content. Above $70 \%$ efficiency the track diameter increases slightly while the hybridization in the track stays roughly constant at $20 \% \mathrm{sp}^{3}$. In graphite a slight increase of the $\mathrm{sp}^{3}$ content can be observed. Track formation starts at about 50\% efficiency. Above $70 \%$ efficiency an increase of $\mathrm{sp}^{3}$ hybridized atoms in the periphery of the track can be observed. We attribute this to a densification of the material due to high pressure in the track center and the comparatively low bulk modulus of graphite.

In contrast to diamond and graphite the amorphous samples show no threshold behavior, but instead have a steady dependence of the radius and coordination decrease of the rearranged volume. This can be attributed to the well-defined dislocation energies in diamond and graphite as opposed to stochastically distributed dislocation energies in the amorphous material.

Diamond and graphite exhibit considerably faster cooling times in the thermal spikes than the amorphous samples (fig. 2.9). In the radial temperature averag- 

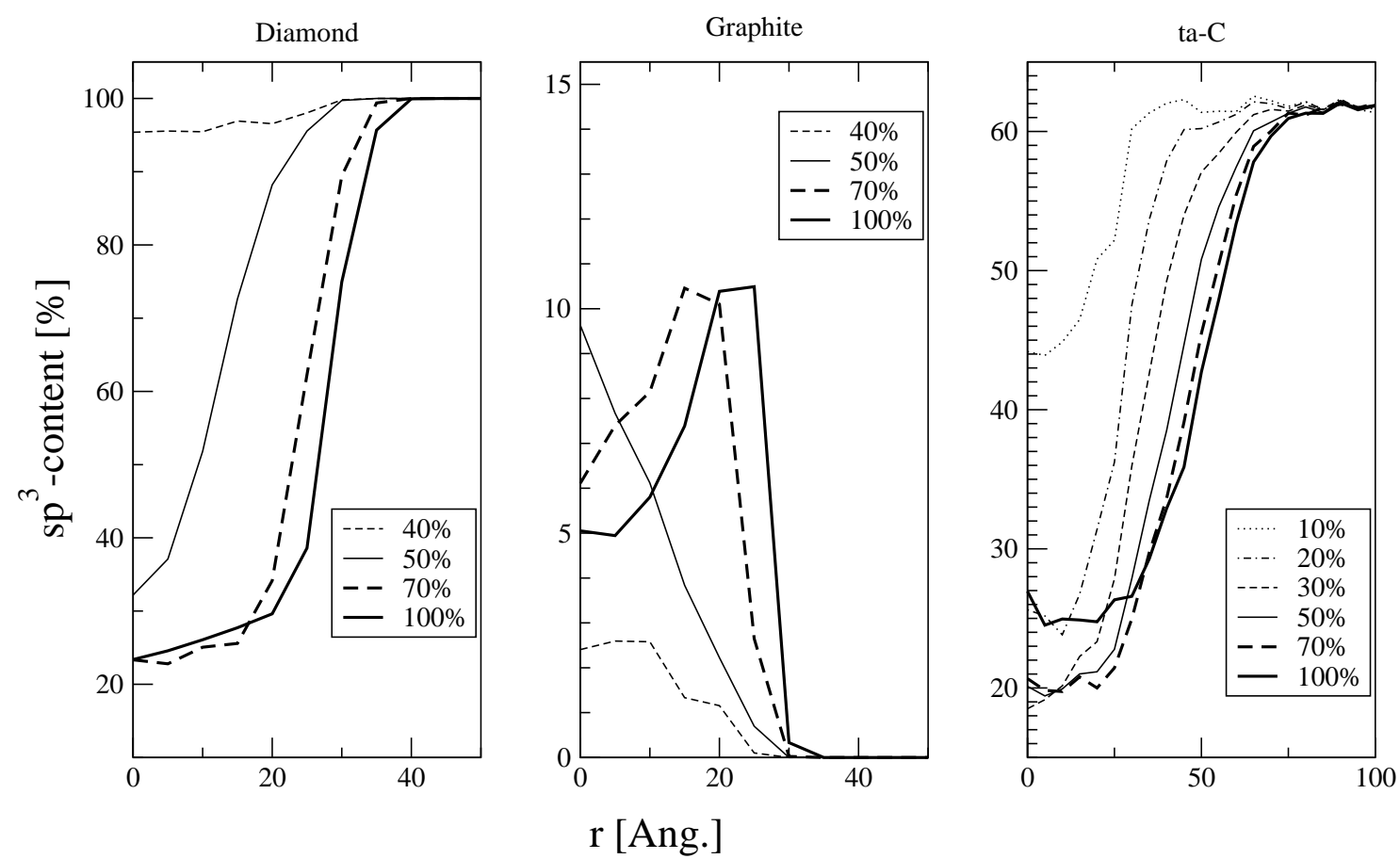

Figure 2.8: Radialy averaged hybridization distributions for selected efficiencies in diamond, graphite, and amorphous carbon. Both diamond and graphite show pronounced differences between the $40 \%$ and $70 \%$ efficiency runs, with an intermediate step at $50 \%$. In diamond the track manifests as a region of decreased $\mathrm{sp}^{3}$ content. Above $70 \%$ efficiency the track diameter increases slightly while the hybridization in the track stays roughly constant at $20 \% \mathrm{sp}^{3}$. In graphite a slight increase of the $\mathrm{sp}^{3}$ content can be observed. Track formation starts at about $50 \%$ efficiency. Above $70 \%$ efficiency an increase of $\mathrm{sp}^{3}$ hybridized atoms in the periphery of the track can be observed. We attribute this to a densification of the material due to high pressure in the track center and the low bulk modulus of graphite. The amorphous samples show no threshold behavior but instead have a steady dependence of the radius and coordination decrease of the rearranged volume. 


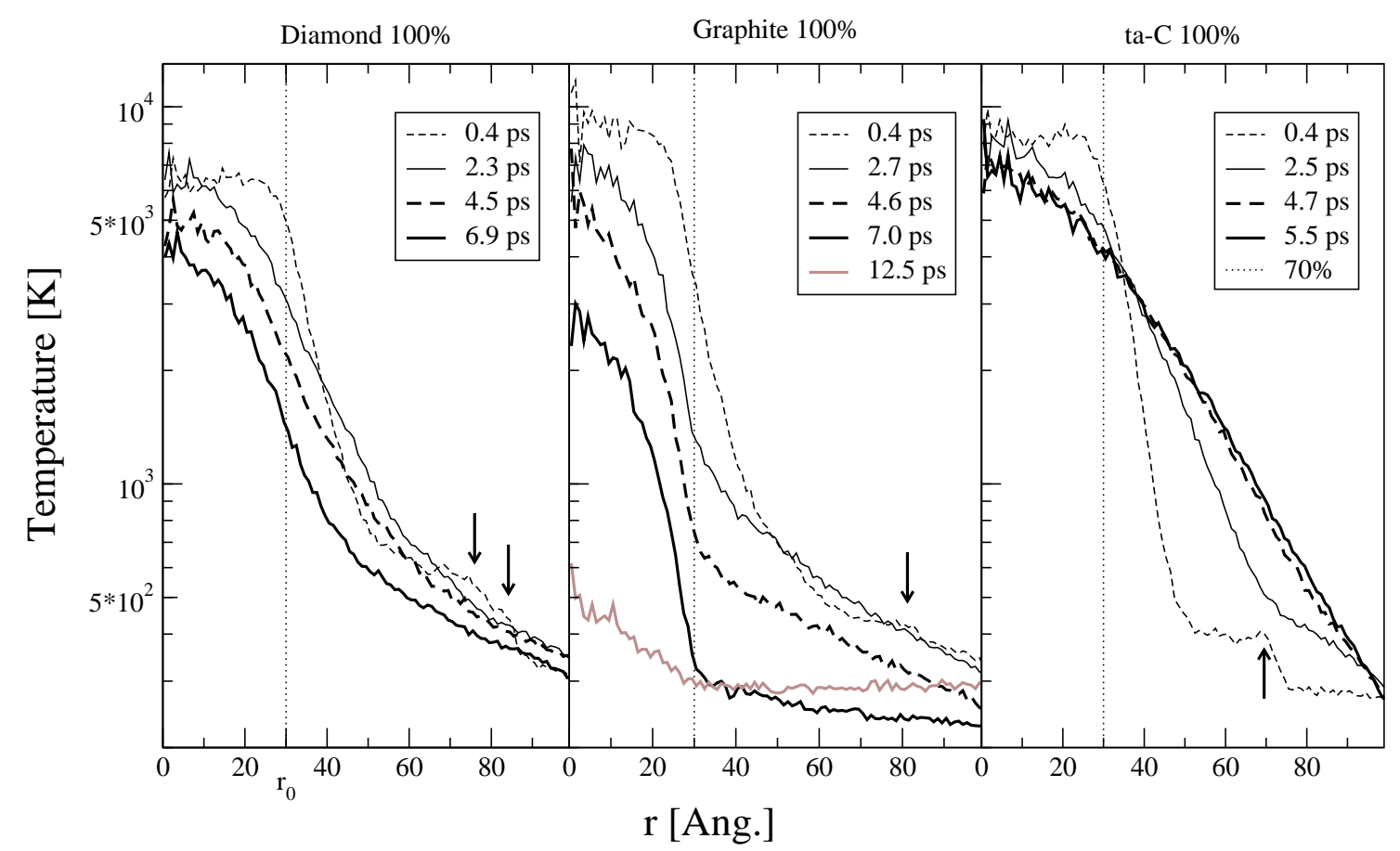

Figure 2.9: Radial temperature distribution in diamond, graphite, and amorphous carbon for maximum efficiency at different times. The horizontal dotted lines mark the extent $r_{0}$ of the initially excited volume. Shockfronts visible in the curves at early times are marked with arrows. 
ing the shockfront in the diamond is smeared out due to anisotropic shock velocities. Both amorphous carbon and graphite along the graphene sheets show isotopic shocks.

\section{Surface effects}

The runs described in the previous section were aimed at simulating bulk effects. Periodic boundary conditions in every direction create infinitely large arrays of evenly spaced infinitely long tracks. The study of the effects of swift heavy ion irradiation on the sample surface morphology requires the omission of periodic boundary conditions in track direction. Without the

\begin{tabular}{c|c|c} 
& $d E / d x[\mathrm{keV} / \mathrm{nm}]$ & $\Delta E[\mathrm{eV} /$ at. $]$ \\
\hline $\mathrm{a}$ & 1.7 & 0.94 \\
$\mathrm{~b}$ & 3.4 & 1.88 \\
$\mathrm{c}$ & 6.7 & 3.75 \\
$\mathrm{~d}$ & 8.4 & 4.69 \\
$\mathrm{e}$ & 10.1 & 5.63 \\
$\mathrm{f}$ & 13.4 & 3.75
\end{tabular}

Table 2.2: Parameters of the free surface simulation runs shown in figure 2.10 . periodicity, the cell size in track direction has to be enlarged substantially. A $48 \mathrm{~nm} \times 48 \mathrm{~nm}$ wide $14 \mathrm{~nm}$ thick amorphous carbon sample with a free surface was prepared and run for six different effective projectile energy losses (table 2.2).

A $48 \mathrm{~nm} \times 48 \mathrm{~nm}$ wide $14 \mathrm{~nm}$ thick amorphous carbon sample with a free surface was prepared and run for six different effective projectile energy losses (table 2.2).

Sections of the final frames of these runs, cut to reveal the track center, are shown in figure 2.10. Periodic boundary conditions were only applied in directions perpendicular to the track. Atoms are colored by kinetic energy with the darkest atoms being the coldest. The extent of the initially excited volume, a cylinder with a radius of $3 \mathrm{~nm}$, is marked with dotted lines. Due to the short lifetime of the molten phase no material transport can occur over long distances. Effectively only a small region near the surface can participate in the hillock formation. In the MD simulation this region is artificially confined by a static layer. All atoms in the lowest $1.5 \mathrm{~nm}$ of the sample are kept at $\vec{v}=0$ and thus frozen in their initial positions. An energy loss dependence of hillock height and width is clearly visible. Very little or no sputtering can be observed at all runs with $d E / d x \leq 10.1 \mathrm{keV} / \mathrm{nm}$. The final 

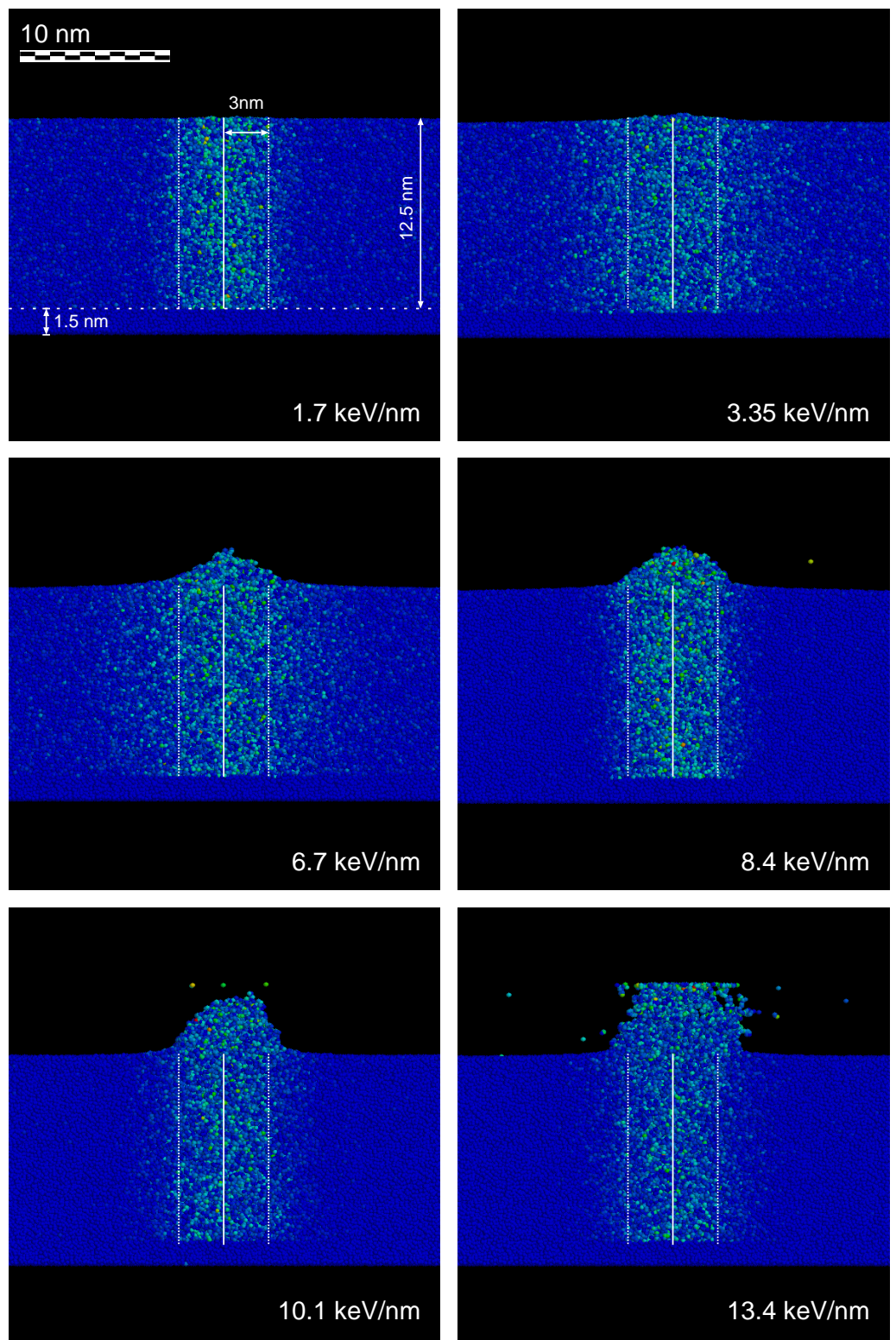

Figure 2.10: Final frames of six simulation runs of a $48 \mathrm{~nm} \times 48 \mathrm{~nm}$ wide $14 \mathrm{~nm}$ thick amorphous carbon sample with a free surface. Periodic boundary conditions were only applied in directions perpendicular to the track. A $1.5 \mathrm{~nm}$ thick bottom layer of atoms was kept static to direct the expansion upwards. Atoms are colored by kinetic energy. The extent of the initially excited volume is marked with dotted lines. An energy loss (table 2.2) dependence of hillock height and width is clearly visible. Very little or no sputtering can be observed at all runs with $d E / d x \leq 10.1 \mathrm{keV} / \mathrm{nm}$. The final run with an effective energy loss of $13.4 \mathrm{keV} / \mathrm{nm}$ leads to an explosion of the hillock with material transport to the upper boundary of the simulation cell. 


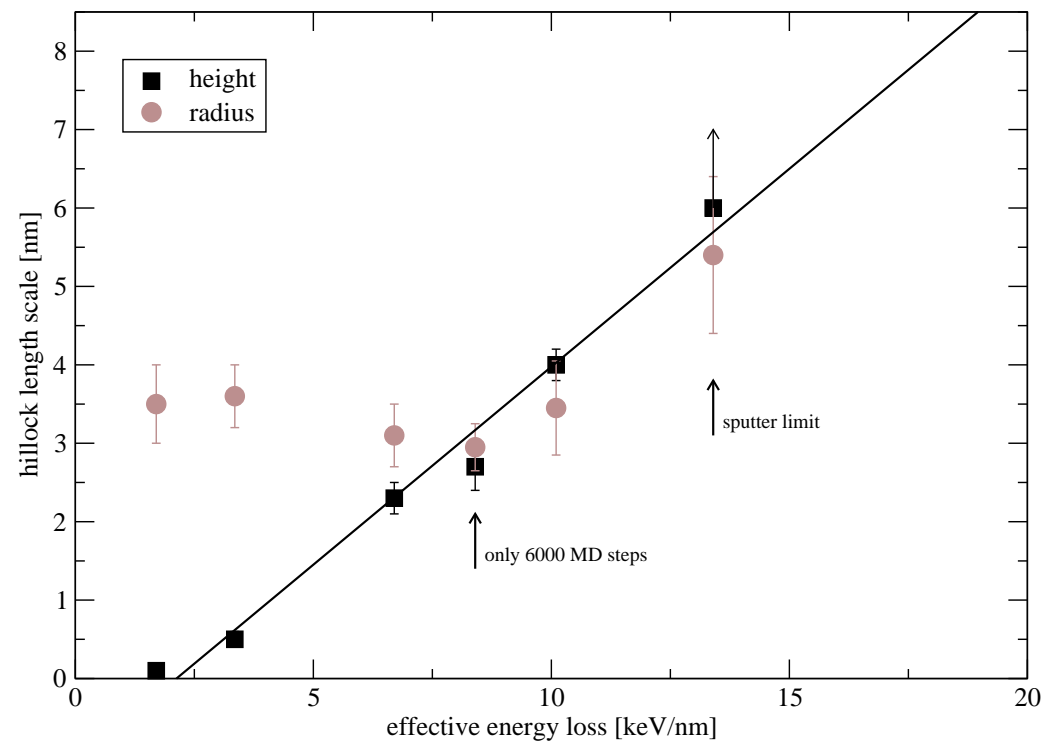

Figure 2.11: Hillock heights and radii from the simulation runs (tab. 2.2). The projectile ions provide the deformation energy for the hillock formation through the effective energy loss. Assuming a linear dependence of hillock volume and deformation energy, the hillock height should scale linearly with the deposited energy if the hillock radius is assumed approximately constant. The assumption holds, as the hillock radii are mainly determined by the fixed $3 \mathrm{~nm}$ radius of the excitation volume. In case of the hillock heights the linear fit shows a good agreement with the hypothesis. The zero crossing at $2.1(1) \mathrm{keV} / \mathrm{nm}$ indicates a threshold energy loss for hillock formation in the simulation. 
run with an effective energy loss of $13.4 \mathrm{keV} / \mathrm{nn}$ leads to an explosion of the hillock with material transport to the boundaries of the simulation cell.

In a simple model the energy needed to form a hillock can be assumed proportional to its volume which scales linearly with height and quadratically with the radius. The hillock radii are mainly determined by the fixed $3 \mathrm{~nm}$ radius of the excitation volume and can be assumed appriximately constant. Thus, for a given energy the height should scale linearly with the deposited energy. Hillock heights (squares) and radii (disks) taken from the simulation runs in table 2.2 are plotted in figure 2.11. A linear fit (black line) describes the data points for the hillock heights reasonably well. The two major deviations from the fit at $6.7 \mathrm{keV} / \mathrm{nm}$ and $13.4 \mathrm{keV} / \mathrm{nm}$ can be explained by an insufficient number of simulation steps and atoms leaving the simulation volume, respectively. The height appears to be a reliable observable to relate the simulation results to experimental data. The zero crossing at $2.1(1) \mathrm{keV} / \mathrm{nm}$ indicates a threshold energy loss for hillock formation in the simulation. Such a threshold was predicted in track formation models [Sze95, Tou92] and observed experimentally in quarz [Mef94] and indium phosphide (InP) [Her98] before.

\subsection{Density of states calculations}

To allow for a comparison of the experimental findings in chapter 3.2 and the molecular dynamics simulation results, ab initio electronic structure calculations were performed. These calculations essentially involve solving the Schrödinger equation for a many-electron system with give atom coordinates. Several computer codes implementing a density functional theoretical (DFT) approach were used. Key concept of DFT is the replacement of the many-electron wavefunction, a function of the $3 N$ electron coordinates ( $4 N$ considering spin), with the electron density, a function of only the 3 spatial dimensions.

Hohenberg-Kohn proved [Hoh64] for any system of interacting particles, the external potential $V$ acting on the particles is uniquely defined to a constant by the ground state particle density $n_{0}$. While the proof didn't specify the nature of these particles, in the following discussion we identify them with electrons. In condensed matter such an external potential contains the coulomb potential of the atomic nuclei. With the potential and the electron density known such an atomic system is 
fully determied, allowing the calculation of excited states as well. Furthermore a functional $E[n]$ can be defined for any potential $V$ calculating the energy for a given electron density. The global minimum of $E$ then constitutes the exact ground state density $n_{0}$.

The first practical self-consistent formalism to calculate this density functional was given a year later with the Kohn-Sham ansatz [Koh65], reformulating the problem to consider a set of independent particles in an effective potential. This effective potential is composed of the original external potential $V$ plus a potential representing the interparticle coulomb forces $e^{2} \int \frac{n\left(r^{\prime}\right)}{r-r^{\prime}} d r^{\prime}$, and a potential $V_{x c}=\frac{\partial E_{x c}[n]}{\partial n}$ representing the exchange-correlation energy $E_{x c}[n]$ of the electrons. The ground state density of the non-interacting particles in the effective potential is exactly the same as the ground state density of the interacting particles in the original potential, provided the exchange-correlation potential is known exactly. As all long-range particle interactions are included in the coulomb potential, $V_{x c}$ is expected to only contain a localized dependence on $n$. Although no analytical way exists to determine $V_{x c}$ and $E_{x c}[n]$, their localized nature allows for a number approximations. The simplest is the local density approximation (LDA), in which the exchangecorrelation energy for a site with density $n$ is taken to be that of a homogeneous electron gas of the same density. Despite its simpliciy, LDA turns out to be surprisingly accurate for a wide range of cases, due to internal cancelation of several error-contributions. LDA applies well to metal-like systems with nearly-free electron gasses. The next approximation step is the generalized gradient approximation (GGA), which includes the local density gradient. However it does not lead to a guaranteed improvement over LDA due to missing error cancelations. Calculations with both approximations have to be compared carefully.

The core electron states are in good approximation independent of the bonding state of the atom. Photoelectron spectroscopy measurements on amorphous carbon films show that the $\mathrm{C} 1 \mathrm{~s}$ orbital located at $230 \mathrm{eV}$ binding energy is shifted by only $1 \mathrm{eV}$ if one of the neighbors is a strongly electronegative fluorine atom. The core electron density is therefore assumed constant and only influences the calculation through screening of the nuclear charge. The nuclear and core-electron potentials can be combined to form an effective potential for the valence electrons, the so called pseudo-potential. 


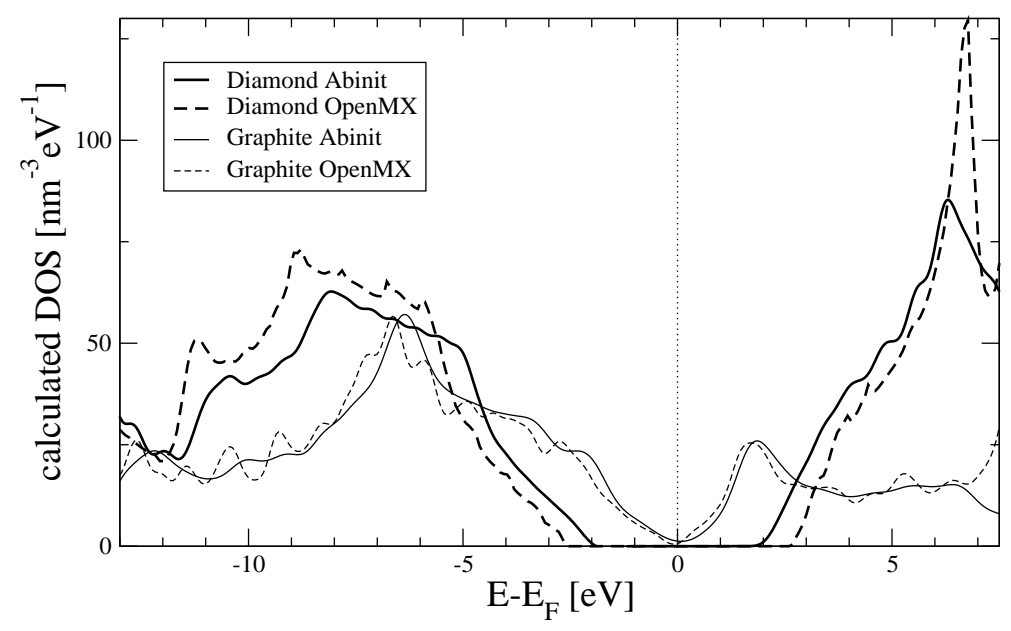

Figure 2.12: Density of states (DOS) for diamond and graphite, calculated using the DFT software packages Abinit [Gon02] and OpenMX [Han06]. Qualitative picture of the bandgap. The Abinit calculation underestimates the diamond bandgap by about $1.5 \mathrm{eV}$. The Fermi energy was shifted to the band gap center.

DFT determines available wavefunctions for the valence electrons in the system. The analytic of these wavefunctions is not known a priori, but it can be formulated in terms of variable coefficients and a base vector set. As the Kohn-Sham formalism is a self-consistent iterative algorithm, care must be taken to ensure the convergence. The presented calculations were performed with a plane wave base set and normconserving Trouiller-Martins-type pseudo-potential [Tro91] using the freely available DFT software packages Abinit [Gon02] and OpenMX [Han06]. For the Abinit calculations a Perdew-Burke-Ernzerhof GGA functional was chosen and the calculation was converged down to one $\mu$ Hartree $(1 \mathrm{Ht}=27.2116 \mathrm{eV})$ total energy variation. The OpenMX calculations were performed using LDA with numerical pseudo atomic basis functions, and converged down to $10^{-10} \mathrm{Ht}$.

DFT results, pictured in fig. 2.12 must be considered qualitative with respect to band-gap widths. Both codes reproduce the semi-metal properties of graphite and the wide band-gap of diamond. However, in the GGA calculation with Abinit the width of the diamond band-gap is underestimated by about $1.5 \mathrm{eV}$. As the KohnSham formalism is a strictly ground state technique, the Fermi level is defined to be the highest occupied state, which coincides with the valence band edge. In fig. 2.12 the Fermi level was manually shifted to the gap center. 
Random samples were cut from empirical potential molecular dynamics simulation frames (see chapter 2.1), from within and outside the affected track regions respectively. The sample volume was chosen to be $(8 \AA)^{3}$ and contained $60-70$ atoms on average. All samples which upon periodic replication yielded atoms closer than $1.2 \AA$ were discarded. To minimize stress under periodic boundary conditions, a tight-binding structure optimization was performed on the remaining samples using the freely available program B-Bop [Oza99]. To perform electronic structure calculations on the relaxed samples the DFT package OpenMX was used, due to the superior results in the diamond and graphite test-calculations.

Computational resources limit the size of the sample volumes, but the application of periodic boundary conditions to the relaxed samples creates quasi crystals. The density of states in the amorphous samples are dominated by a small volume, representing the entire amorphous track or matrix. A few localized states will dominate the DOS, therefore the density of state histograms of many samples were configurationally averaged [Ban89]. Figure 2.13 shows these configuration-averaged DOS for track (top) and unirradiated a-C matrix (bottom). The unshifted DOS of diamond (solid grey line) and graphite (dashed grey line) from fig. 2.12 are added to the plots for comparison. Similarities of the track and matrix DOS to the graphite and diamond DOS respectively are apparent. As a first approximation the a-C spectra appear to be a linear combination of the graphite and diamond DOS. Effects going beyond a simple linear combination can be attributed to energy level shifts due to bond strain.

\subsection{TEM image simulation}

To facilitate the comparison of MD simualation results with transmission electron microscopy (TEM) images of irradiated carbon samples, an image simulation algorithm was implemented.

The basic multislice formalism [Cow57] was chosen for its simplicity and applicability to the studied samples. Its basic concept is the decomposition of the three-dimensional electrical potential distribution in the sample into a set of twodimensional potential slices. These slices are treated sequentially as the electron wavefront propagates through the sample. 

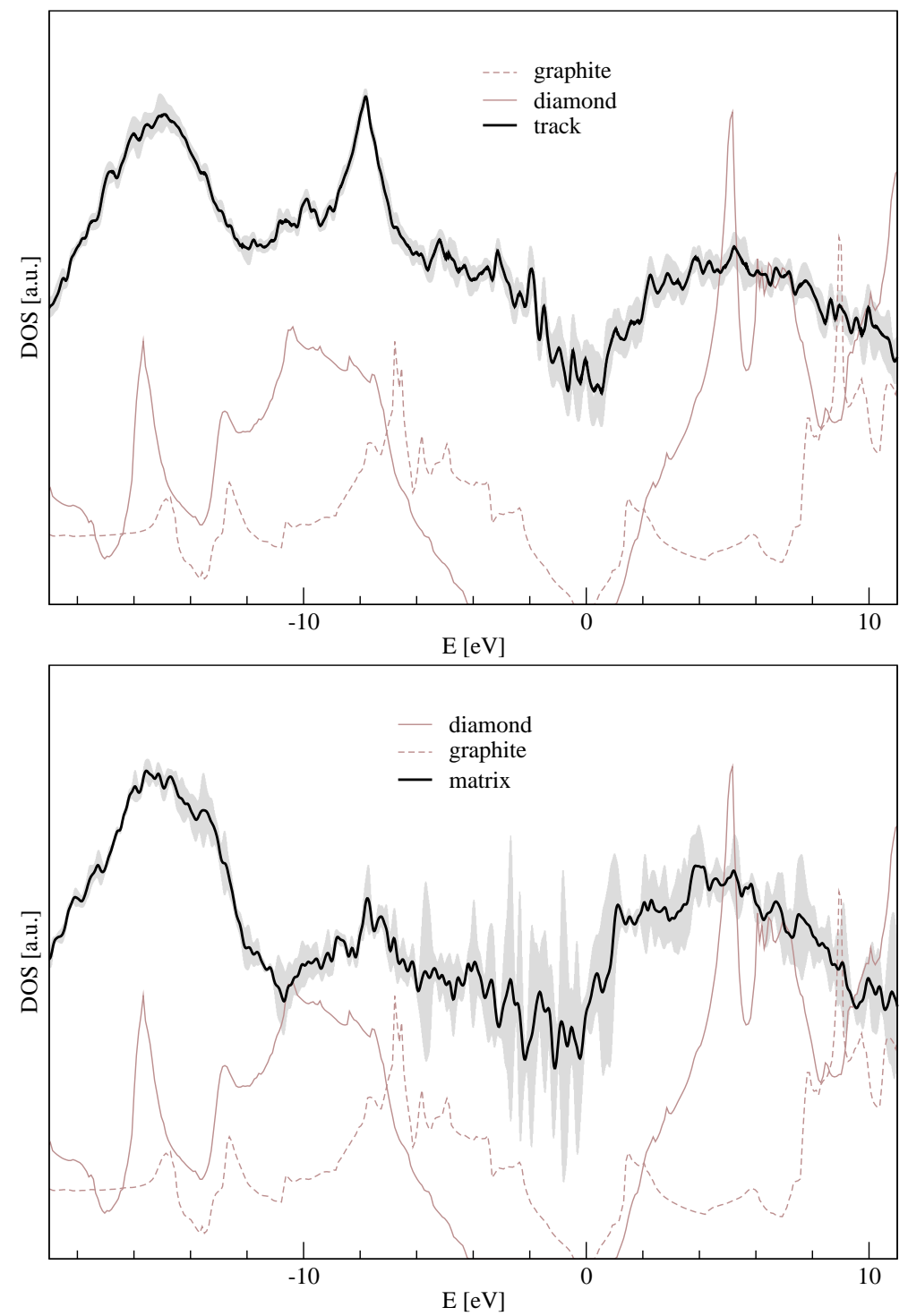

Figure 2.13: Configuration-averaged density of states from inside an ion-track (top) and unirradiated a-C (bottom), plotted as black curves with gray error margin, compared to the DOS of diamond (solid grey line) and graphite (dashed grey line). Several DOS of $(8 \AA)^{3}$ samples taken from empirical potential molecular dynamics simulations were averaged to obtain the curves. The samples were relaxed using the tight-binding code b-bop [Oza99] with periodic boundary conditions applied. DOS calculations were performed with OpenMX [Han06]. Similarities of the track and matrix DOS to the graphite and diamond DOS respectively are apparent. As a first approximation the a-C spectra appear to be a linear combination of the graphite and diamond DOS. 
The accuracy of this formalism is comparable with calculations based on the dynamical theory of Bethe [Bet28]. Due to an implicit small angle approximation [Ale97] for the electron scattering angles it can only be applied for elecron beam energies above $50 \mathrm{keV}$. In this study all TEM imaged were acquired using a $200 \mathrm{keV}$ field emission gun TEM.

Only elastically scattered electrons are considered in this formalism as only the potential and not the underlying charged particles as sources of the potential are treated. In practice the electrons may undergo inelastic collisions. However, inelastically scattered electrons are incoherent and do not contribute local structure information.

Trajectory simulations for carbon films similar to the analyzed samples were carried out with the Monte Carlo software Casino v2.42 to estimate the amount of inelastically scattered electrons. The simulations were run with an electron energy of $200 \mathrm{keV}$, the TEM acceleration energy used for imaging.

Only a small fraction of electrons is scattered

\begin{tabular}{l|c|c} 
& $2.7 \mathrm{~g} / \mathrm{cm}^{3}$ & $3.1 \mathrm{~g} / \mathrm{cm}^{3}$ \\
\hline $50 \mathrm{~nm}$ & $0.2 \%$ & $0.3 \%$ \\
\hline $100 \mathrm{~nm}$ & $0.6 \%$ & $0.7 \%$
\end{tabular}

Table 2.3: Fraction of electrons scattered off the primary beam axis in carbon films of different thickness and density. Simulation performed with Casino v2.42 for electron energies of $200 \mathrm{keV}$ using the default physical model settings. off the primary beam axis for either density and thickness (table 2.3), indicating that the amplitude contrast between matrix material and track core in the bright field (BF) image plays a negligible role.

This is in agreement with the elastic mean free path $\lambda_{\text {el }}>150 \mathrm{~nm}$ for $200 \mathrm{keV}$ electrons in amorphous carbon, calculated using semiempirical formulas based on electron energy loss spectroscopy (EELS) data [Vos05, Mal88].

A computer program was written to implement the multislice formalism (fig. 2.14) in the fast fourier transformation (FFT) formulation. The program calculates the contrast transfer function and Fresnel propagator from microscope parameters and creates a wavefunction for the incoming plane electron wave. This incoming wave is successively phase-modulated and propagated at each potential slice, according to

$$
\psi_{n+1}(\vec{k})=\mathcal{F}\left(\mathcal{F}^{-1}\left(\psi_{n}(\vec{k}) \cdot P\right) \cdot q_{n+1}(x)\right),
$$

with $\mathcal{F}$ being the fourier transform operator, $\psi_{n}$ the electron wave in reciprocal space, $q_{n}$ the atomic potential of slice $n$ in real space. Propagation inside the 


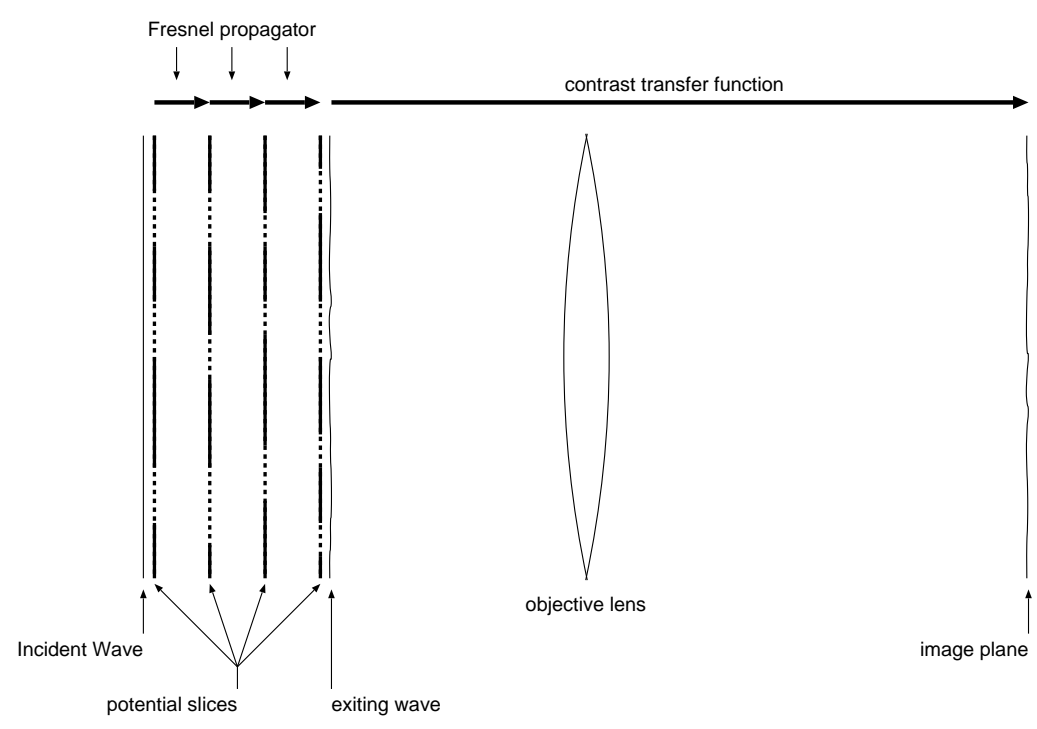

Figure 2.14: Schematic diagram of the components needed for a multislice TEM image simulation. The incident wave is phase-modulated at each potential slice and propagated by the Fresnel propagator as if it were traveling in empty space. The exiting wave in the object plane is then convolved with the contrast transfer function (CTF), which models the effect of the objective lens and the space in between object and image planes.

sample, between slices, is approximated by a wave propagation in vacuum. This is described by the Fresnel propagator $P$, which contains the microscope acceleration voltage in terms of electron wavelength. The iteration process over all sample slices generates the exiting wave in the object plane.

The information transfer from the object plane to the image plane is governed by the contrast transfer function (CTF). The CTF oscillates between +1 and 1 as a function of the spatial frequency, with the positions of the zero crossings depending on the defocus length. At zero points of the CTF no object information is transmitted to the image. This omission of spatial frequencies creates the phase contrast. The CTF of an ideal mircoscope at zero defocus would be unity. Thus, such a microscope would only exhibit amplitude contrast, as all phase information vanishes during image formation. However, it has long been known [Zer42] from light microscopy that phase contrast can be increased by imaging with an applied defocus. 


\subsubsection{Averaged potential image simulation}

As a first approximation, the atomic potential can be averaged along the electron trajectories inside the sample. For an ideally amorphous sample this mean potential would be completely uniform. This uniform potential is modulated with the radial density distribution perpendicular to the ion tracks.

The atomic potential slows down electrons traveling through the sample by shifting fractions of their kinetic energy to potential energy. As the kinetic energy reduction depends on the local density, this effect induces phase shifts between electrons passing through different points in the sample.

The mean potential in the carbon samples can be calculated by performing a volume integration over the potential of a single carbon atom and multiplying the result with the atomic density in the sample. As an approximation, the atomic potential of a free carbon atom is taken, calculated from the Doyle-Turner coefficients given in $[$ Doy67]

$$
V_{A}(\vec{r})=\frac{\hbar^{2}}{(2 \pi)^{2} m_{0} q_{e}} \cdot \sum_{i=1}^{4} A_{i} \cdot\left(\frac{4 \pi}{B_{i}}\right)^{\frac{3}{2}} \cdot e^{-\frac{r^{2}}{B_{i}}}
$$

with

$$
\begin{aligned}
& A_{i}=0.7307,1.1951,0.4563,0.1247 \\
& B_{i}=35.9951,11.2966,2.8966,0.3456 .
\end{aligned}
$$

The integration of the $r$ dependent term yields

$$
\int e^{-\frac{x^{2}+y^{2}+z^{2}}{B_{i}}} d^{3} r=\pi^{\frac{3}{2}} B_{i}^{\frac{3}{2}} \quad, \quad B_{i}>0
$$

allowing for a cancelation of the $B_{i}$ from the integral. Substitution of the coefficients results in a value of $120.02 \mathrm{~V}^{3}$ for the volume potential. With the atomic densities of graphite $\rho_{g}=\frac{4}{35.189} \AA^{-3}$, and diamond $\rho_{d}=\frac{8}{45.385} \AA^{-3}$, the respective mean potentials can be calculated as

$$
\begin{aligned}
& V_{g}=\rho_{g} \cdot 120.02 \mathrm{VA}^{3} \approx 13.6 \mathrm{~V} \\
& V_{d}=\rho_{g} \cdot 120.02 \mathrm{VA}^{3} \approx 21.2 \mathrm{~V} .
\end{aligned}
$$



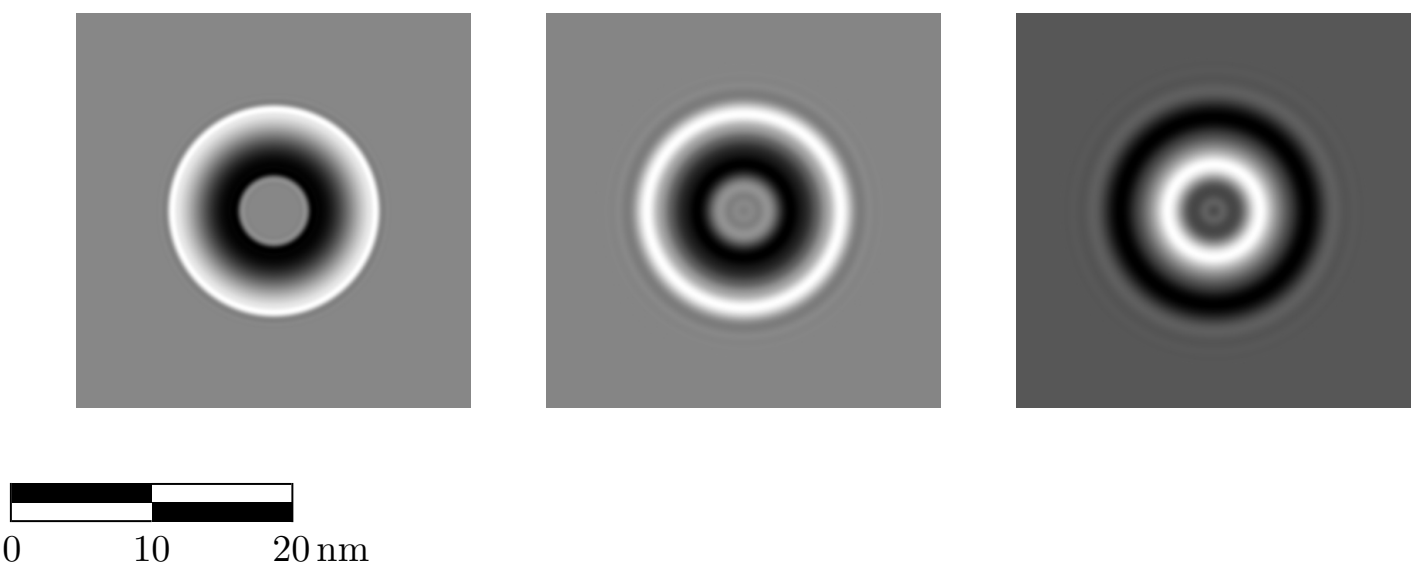

Figure 2.15: Three simulated TEM images with defocus settings $0 \mu \mathrm{m}, 3.6 \mu \mathrm{m}$ and $-7.5 \mu \mathrm{m}$. The images have been normalized to yield maximum contrast. The difference between highest and lowest electron wave intensity are $20 \%$ for $3.6 \mu \mathrm{m}$ defocus and $30 \%$ for $-7.5 \mu \mathrm{m}$. At zero defocus (left) the only source of phase contrast are the Fresnel propagations, resulting in an intensity contrast ten orders of magnitude below the defocussed images, and thus technically undetectable.

The density of an amorphous carbon material $\rho_{a}$ can be interpolated [Hof98] from the diamond and graphite densities, depending on the atomic $\mathrm{sp}^{3}$ hybridization fraction $s$ with the expression

$$
\rho_{a}=s \cdot \rho_{d}+(1-s) \cdot \rho_{g}
$$

To model the radial density distribution of the ion tracks for the simulation $s$ was defined as a function of the distance $r$ from the track center. $s(r)$ is a piecewise composition of constant densities inside $\left(r<\sigma_{1}\right)$ and outside $\left(r>\sigma_{2}\right)$ the track, and a smooth connecting function in between.

$$
s(r)=\left\{\begin{array}{cl}
0 & r<\sigma_{1} \\
1-\cos ^{2}\left(\frac{\pi}{2} \frac{r-\sigma_{1}}{\sigma_{2}-\sigma_{1}}\right) & \sigma_{1}<r<\sigma_{2} \\
1 & r>\sigma_{2}
\end{array}\right.
$$

Figure 2.15 shows three simulated TEM images with defocus settings $0 \mu \mathrm{m}, 3.6 \mu \mathrm{m}$ and $-7.5 \mu \mathrm{m}$. The track geometry is determined by the shape parameters $\sigma_{1}=$ $2.5 \mathrm{~nm}$ and $\sigma_{2}=7.5 \mathrm{~nm}$, resulting in a track FWHM $10 \mathrm{~nm}$, with $20 \% \mathrm{sp}^{3}$ in the track and $60 \% \mathrm{sp}^{3}$ in the matrix. All resulting images were normalized, with the miniumum intensities appearing black and the maximum intensities appearing white. The difference between highest and lowest electron wave intensity are $20 \%$ 
for $3.6 \mu \mathrm{m}$ defocus and $30 \%$ for $-7.5 \mu \mathrm{m}$. At zero defocus the only source of phase contrast are the Fresnel propagations. The resulting pattern is an image of the second derivative of the potential and possesses an intensity contrast ten orders of magnitude below the defocussed images, making it practically undetectable.

\subsubsection{High resolution image simulation}

The multislice formalism can also be used for the simulation of high resolution images, if a potential distribution based on individual atomic coordinates is used for input rather than a long-range averaged potential.

Such potential maps were generated from MD datasets. Again as an approximation the electron density distribution was taken as the sum of free carbon atom electron densities calculated using the Doyle-Turner potential [Doy67]. This approximation is reasonable, as the electron density modulation due to the formation of covalent bonds is low compared to the total electron density [Mar05]. Equation 2.3 was evaluated and summed up on a three-dimensional grid for every atom in the sample. The chosen grid size of $1024 \times 1024 \times 344$ points, corresponding to a sample size of $22.2 \times 22.2 \times 7.4 \mathrm{~nm}^{3}$, yields a spacial resolution of about $0.2 \AA$. For the evaluation, the same periodic boundary conditions used in the initial MD simulation were applied.

Key advantage of this method is a more natural looking simulation result which is immediately comparable to the actual TEM images. During TEM image acquisition, the contrast and brighntness settings are adjusted by the operator, to yield optimum image contrast. When adjusting the contrast of the simulated image, the signal generated by the amorphous matrix can be used as a normalizing factor.

Figure 2.16 shows three simulated TEM images with atomic potential distribution generated from the final frame at $6.2 \mathrm{ps}$ of the $90 \%$ efficiency amorphous carbon simulation. The defocus settings are $0 \mu \mathrm{m}, 3.6 \mu \mathrm{m}$, and $-7.5 \mu \mathrm{m}$. At zero defocus the track is barely visible. The contrast transfer function provides no contrast at length scales $>2 \mathrm{~nm}$.

The periodicity in the images results from the periodicity in the amorphous starting sample which was assembled from a small amorphous building block. This building block has an irregular density distribution which gets amplified by the periodic 

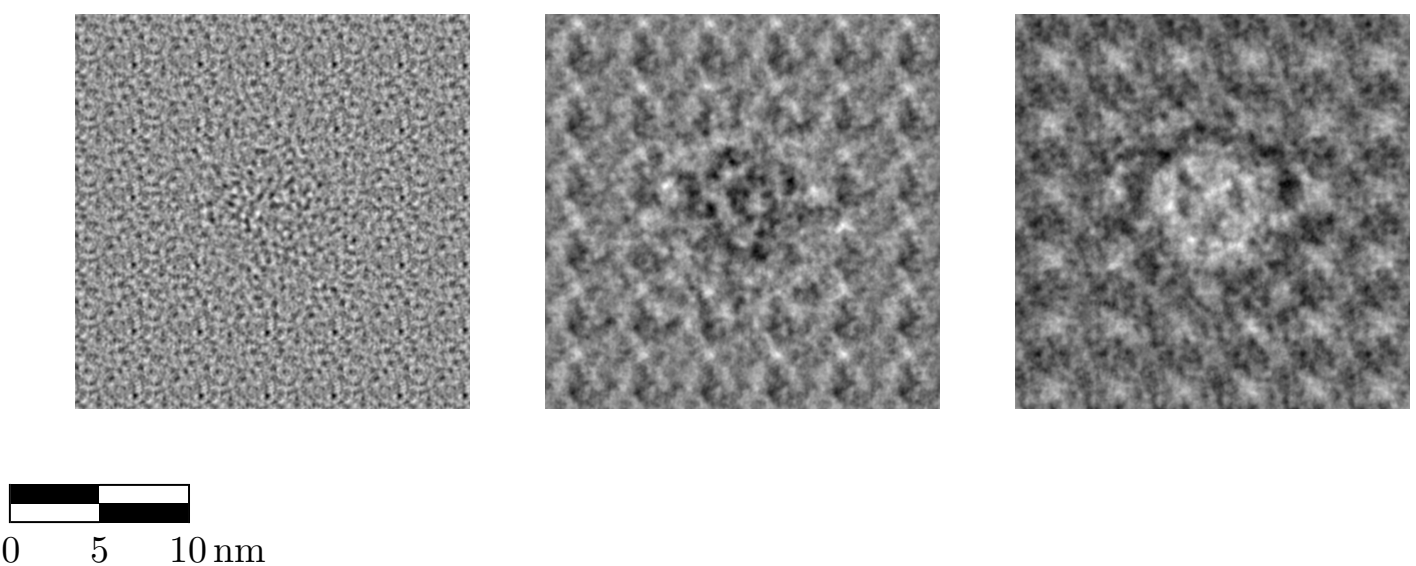

Figure 2.16: Three simulated TEM images, from a-C MD data, with defocus settings $0 \mu \mathrm{m}$, $3.6 \mu \mathrm{m}$, and $-7.5 \mu \mathrm{m}$. At zero defocus the track is barely visible. The periodicity in the images results from the periodicity in the amorphous starting sample which was assembled from an amorphous building block. This building block has an irregular density distribution which gets amplified by the periodic replication, creating a pronounced image contrast which competes with the track contrast. All images have been normalized to exhibit maximum contrast.

replication, creating a pronounced image contrast which competes with the track contrast. Future simulations with fully amorphous starting samples are planned to address this issue. The track contrast is expected to show up more pronounced in those images.

The potential of the image simulation becomes more apparent when applied to the simulation in diamond. Close to the track creation threshold the track sizes strongly depend on the effective energy loss (see fig.2.5). Figure 2.17 shows the simulated TEM images for different amorphized track sizes. The rows correspond to $3 \mathrm{~nm}$, $4.5 \mathrm{~nm}$, and $6 \mathrm{~nm}$, with the efficiencies (effective energy losses) of $50 \%(7.5 \mathrm{keV} / \mathrm{nm})$, $70 \%(10.5 \mathrm{keV} / \mathrm{nm})$, and $100 \%(15.0 \mathrm{keV} / \mathrm{nm})$. The columns correspond to defocus settings $0 \mu \mathrm{m},-7.5 \mu \mathrm{m}$, and $3.6 \mu \mathrm{m}$.

The tracks exhibit a high phase contrast against the featureless crystalline matrix. As the image depends mainly on the density distribution, the amorphous carbon simulations with a truly amorphous matrix are expected to exhibit a similar contrast pattern. 

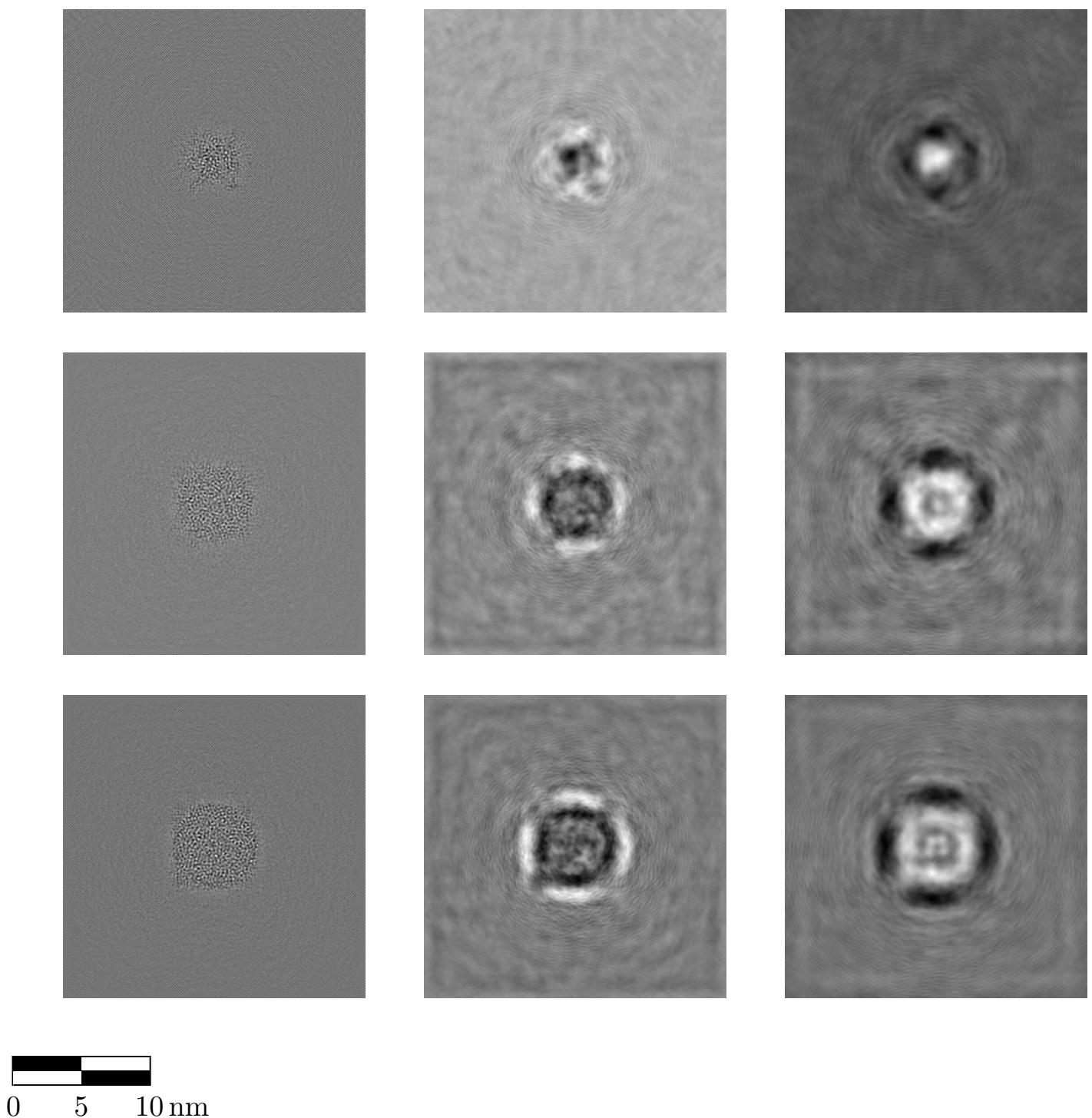

Figure 2.17: $\quad$ Simulated TEM images of diamond track samples created with $50 \%$ efficiency (top row), and $70 \%$ efficiency (bottom row). The defocus settings are $0 \mu \mathrm{m}, 3.6 \mu \mathrm{m}$, and $-7.5 \mu \mathrm{m}$. The influence of the track size on the final image is clearly visible, the track diameters (top to bottom) are $3 \mathrm{~nm}, 4.5 \mathrm{~nm}$, and $6 \mathrm{~nm}$ (fig. 2.5), resulting in diffration pattern diameters of about $5 \mathrm{~nm}, 6.5 \mathrm{~nm}$, and $7.5 \mathrm{~nm}$. 


\section{Chapter 3}

\section{Experimental results}

\subsection{Atomic force microscopy}

The surface topography of carbon samples before and after irradiation was analyzed using atomic force microscopy (AFM) [Bin86]. This scanning probe technique utilizes the interaction of the sample with a sharp tip attached to a micro mechanical cantilever. Forces between the tip an the sample surface cause a bending of the cantilever, which is measured optically by detecting the reflection of a laser beam with a multisegment photodiode. Apart from measuring topography through orbital repulsion, electrostatic or magnetic forces can be measured by changing the tip composition. Several image acquisition modes can be employed, depending on the sample morphology and desired data.

In contact-mode the sample topography is scanned with the cantilever constantly in contact with the surface. Height information can be gathered by measuring the cantilever curvature directly. However, to avoid the non-linear relation of curvature and surface height, a control loop can be used to keep the curvature constant by adjusting the scanner position perpendicular to the sample surface. Due to the constant contact friction occurs between tip and sample surface. This friction can lead to abrasions and damage of soft samples. However, it presents an additional measurable quantity, the lateral force, which leads to cantilever torsion deflecting the laser beam perpendicular to the axis of bending. Thus, lateral force microscopy maps the friction between tip and sample. 


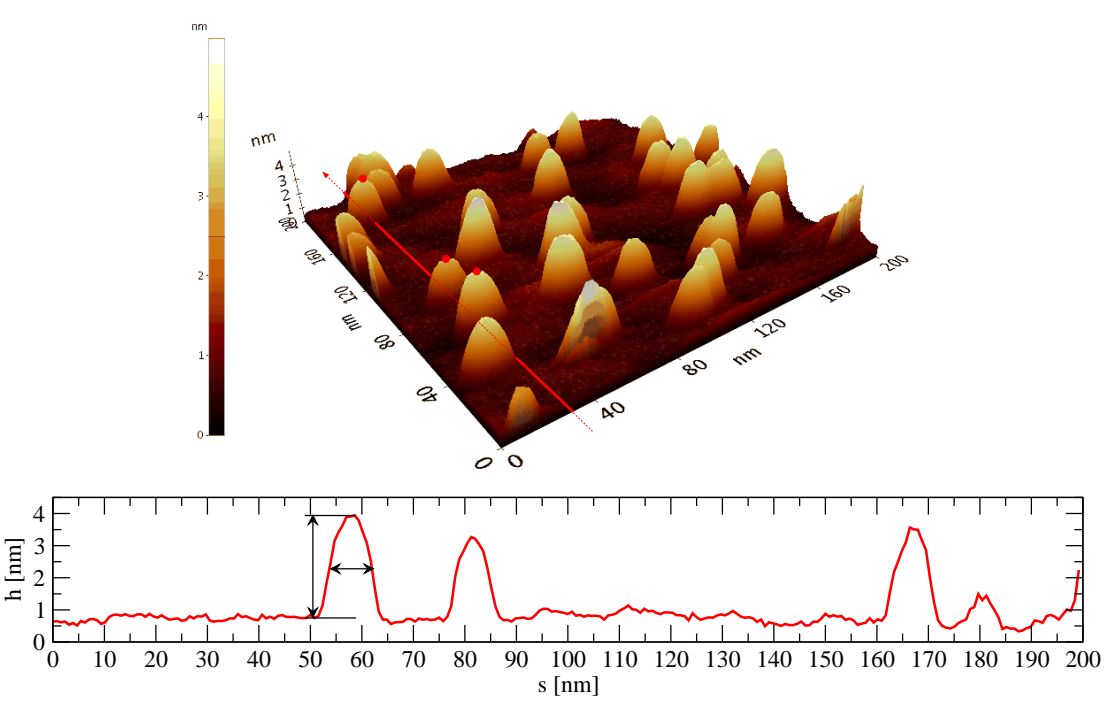

Figure 3.1: Top: Contact mode topography AFM image of a ta-C sample grown on silicon, irradiated with $10^{11} \frac{\mathrm{ions}}{\mathrm{cm}^{2}}$. The depicted area of $200 \times 200 \mathrm{~nm}^{2}$ exhibits 37 hillocks. On average 40 ion impacts are expected. The hillocks only appear on irradiated samples and their area density corresponds to the ion fluence within statistical limits. This suggests a 1:1 correspondence of hillocks and ion impacts. Bottom: Linescan along the white line marked in the above image. Hillock height is about $3 \mathrm{~nm}$ and width at half maximum is about $8 \mathrm{~nm}$.

In noncontact-mode the cantilever is actuated as a driven oscillator slightly above the resonance frequency. Attractive dispersion forces (van der Waals) effectively lower the reset force of the oscillator as a function of the sample-tip distance, lowering its resonance frequency. A control loop is employed to keep the oscillation amplitude constant by adapting the actuation frequency. Due to the long range of the participating forces the resolution is reduced in this mode. However, it is the most sparing AFM mode and thus suitable for soft biological samples.

In tapping-mode, or intermediate-mode, the phase shift between the actuation signal and the cantilever oscillation can be measured, yielding a dampening factor depending on sample elasticity. Chemical functionalization of the tip allows to map specific chemical properties of the sample surface, through phase-shifts caused by local stickiness of the surface [Noy97].

All images in this work were acquired in air at room temperature using a PSIA XE-100 in contact mode, unless stated otherwise. 
The top part of fig. 3.1 shows a contact mode topography AFM image of a ta-C sample grown on silicon, irradiated with $10^{11} \frac{\text { ions }}{\mathrm{cm}^{2}} 1 \mathrm{GeV}$ lead $(\mathrm{Pb})$ ions. The depicted area of $200 \times 200 \mathrm{~nm}^{2}$ exhibits 37 hillocks. On average 40 ion impacts are expected. The hillocks only appear on irradiated samples and their area density corresponds to the ion fluence within statistical limits. This suggests a 1:1 correspondence of hillocks and ion impacts. The bottom part shows a linescan along the white line marked in the above image. Hillock height is about $3 \mathrm{~nm}$ with a width at half maximum of about $8 \mathrm{~nm}$.

Piezo drift, sample curvature, and tilt dominate the topography of a raw AFM image. To emphasize the local structure these contributions have to be carefully removed through a process called flattening. In a first step the image was flattened line wise along the fast scanning direction by subtracting a second order fit from the height information. Surface features, such as hillocks, increase the average height in a scan line, effectively lowering the substrate level after flattening. To remove this artifact in a second step all hillocks were masked out, leaving just the pristine carbon film areas, and another flattening step was applied.

\subsubsection{Conducting atomic force microscopy}

By using cantilevers coated with an electrically conducting platinum iridium (PtIr) alloy and a bias voltage applied to the sample a spatially resolved current map can be acquired simultaneously to the topography data in contact-mode. Such a conducting atomic force microscope (C-AFM) allows the measurement of the electrical properties of single ion tracks. With the scanning motion halted the cantilever can be positioned and kept at defined spots with a few nanometer accuracy, permitting local IV spectroscopy.

Figure 3.2 shows current-voltage curves measured at room temperature on individual tracks and on unirradiated spots between tracks for two samples with different irradiation densities. The conductivity of the ion tracks (on track) is about four magnitudes higher than of the surrounding ta-C matrix (off track). The data, acquired at room temperature, was fit assuming a Frenkel-Poole conduction mechanism (dashed lines). Deviation from the fit can be attributed to capacitance of the sample holder and contact resistances. 

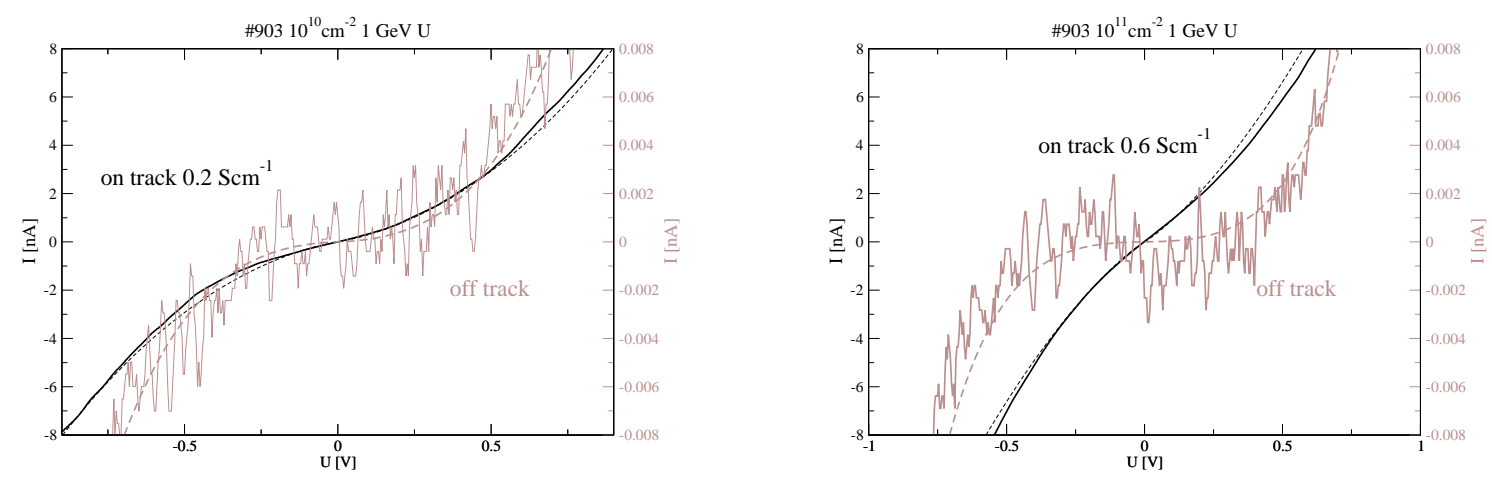

Figure 3.2: Comparison of I-U-curves on- and off-track for two samples with different irradiation densities measured with an AFM under air. The conductivity of the ion tracks (on track) is about four magnitudes higher than of the surrounding ta-C matrix (off track). All data was fit assuming a Frenkel-Poole conduction mechanism (dashed lines). Deviation from the fit can be attributed to capacitance of the sample holder and contact resistances.

\subsubsection{Statistical hillock-height analysis}

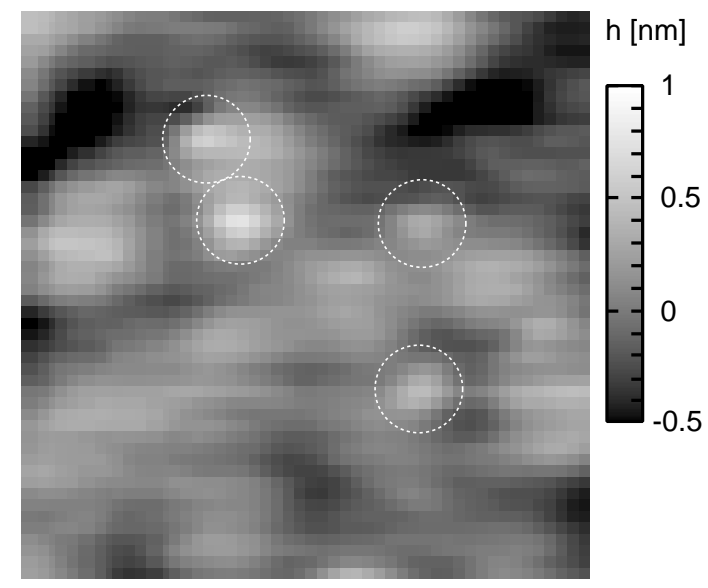

Figure 3.4: High-pass filtered AFM topography of a xenon (Xe) irradiated ta-C film with low-frequency film roughness partially removed to enhance the hillock signal.
A series of iradiations was performed to investigate the hillock height as a function of the electronic energy-loss $d E / d x$ (fig. 3.3). Through the use of various projectile ion species and energies the stopping power range from about $12 \mathrm{keV} / \mathrm{nm}$ to $70 \mathrm{keV} / \mathrm{nm}$ could be sampled. Only irradiation runs with degrader foils (dark circles) were included in this study. Previous irradiations without degraders (light circles) were disregarded, as the charge state of the ions directly after acceleration differs too much from the equilibrium charge state of the moving ions in matter. Upon entry in a solid the swift ions are stripped of electrons until the increasing electrical charge of the ions causes the rate for capturing of electrons from the solid to exactly compensate for the stripping. As the stopping power is very sensitive to the charge state the lack of stripping by 


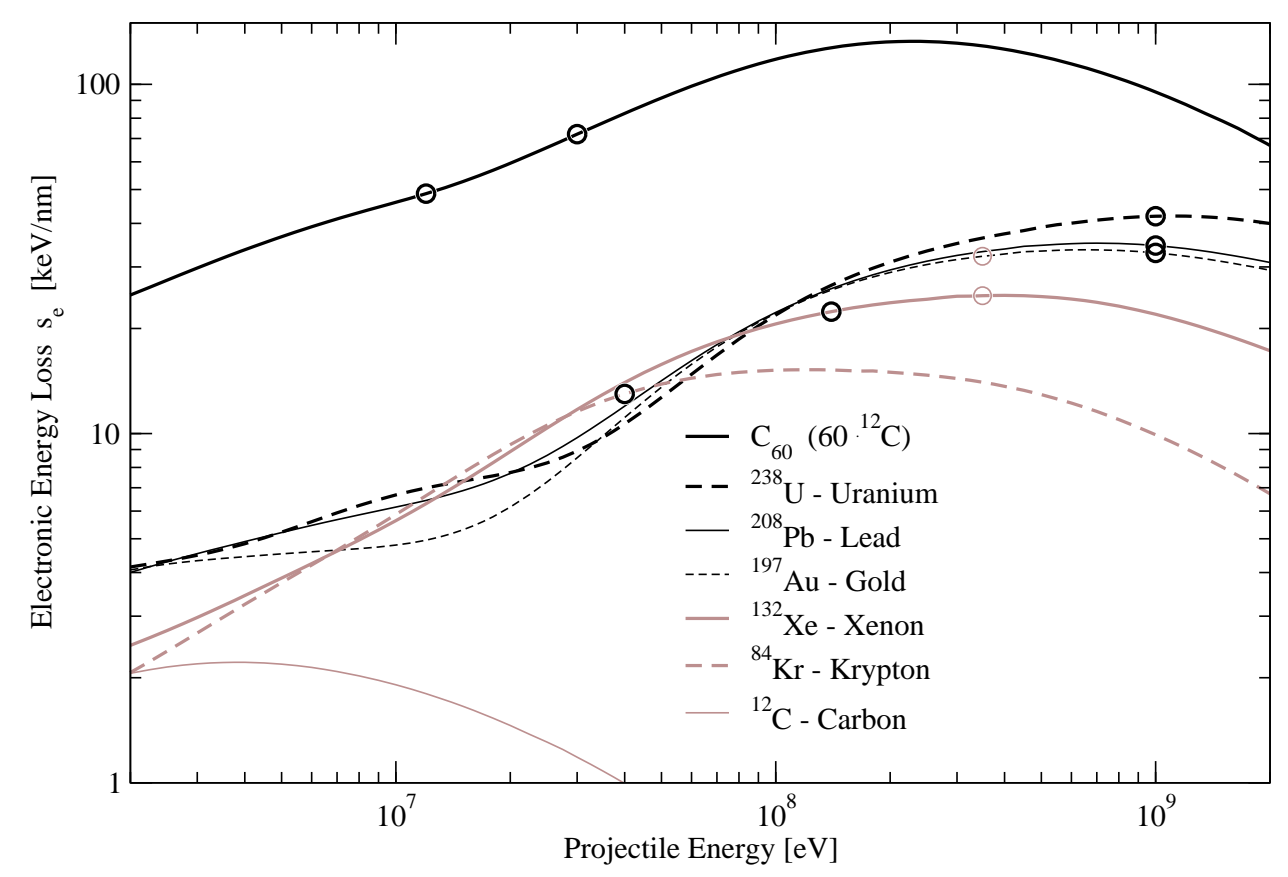

Figure 3.3: Electronic stopping power curves in amorphous carbon, for all ion species selected for irradiations, computed using the Monte-Carlo software SRIM2003 [Zie85]. Dark circles mark irradiation runs with degrader foils, light circles mark irradiation runs without degrader foils. The $\mathrm{C}_{60}$ curve is computed by summing the stopping powers of its constituent atoms, disregarding potential coherent stopping effects. 


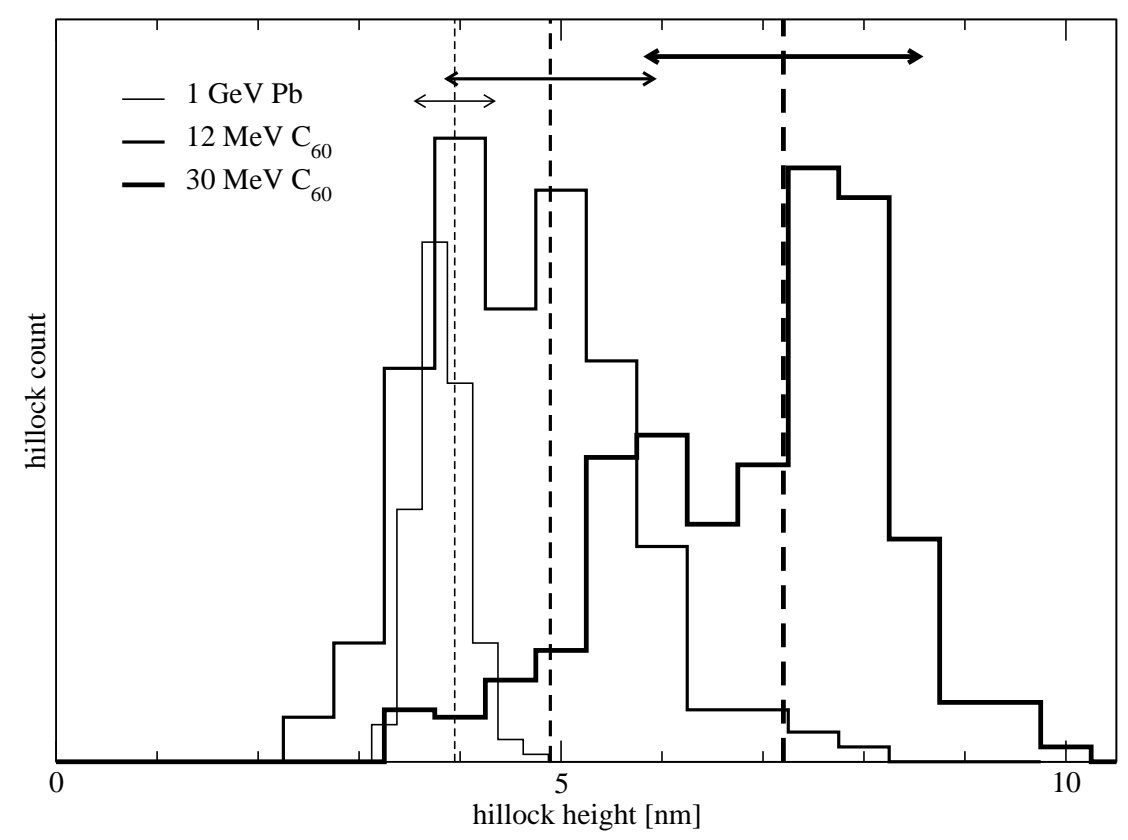

Figure 3.5: Height distribution of hillocks on samples irradiated with swift heavy ion species and energies.

the degrader foils would cause a reduced stopping power in the surface region of the tracks.

To achieve stopping powers well in excess of $40 \mathrm{keV} / \mathrm{nm} \mathrm{C}_{60}$ cluster-ion irradiation was performed. The stopping power curve for the $\mathrm{C}_{60}$ ions was computed by summing the stopping powers of its constituent atoms, disregarding potential coherent stopping effects [Jen00].

To avoid human bias an automatic hillock detection and height measurement computer algorithm was designed and implemented in C. The resulting program reads a flattened AFM image and finds the location of maximum height. In any flattened, artifact- and adsorbate-free image it is safe to assume that this point corresponds to the peak of a hillock. It then outputs the hillock height, which is defined as the difference between its peak height and the minimum height on a circular annulus with the radius $r_{a}$ centered on the peak. $r_{a}$ was adapted to always encompass the convolved image of tip and hillock. All data points within $r_{a}$ are removed and the preceding steps are repeated until $N$ hillock heights are determined. 


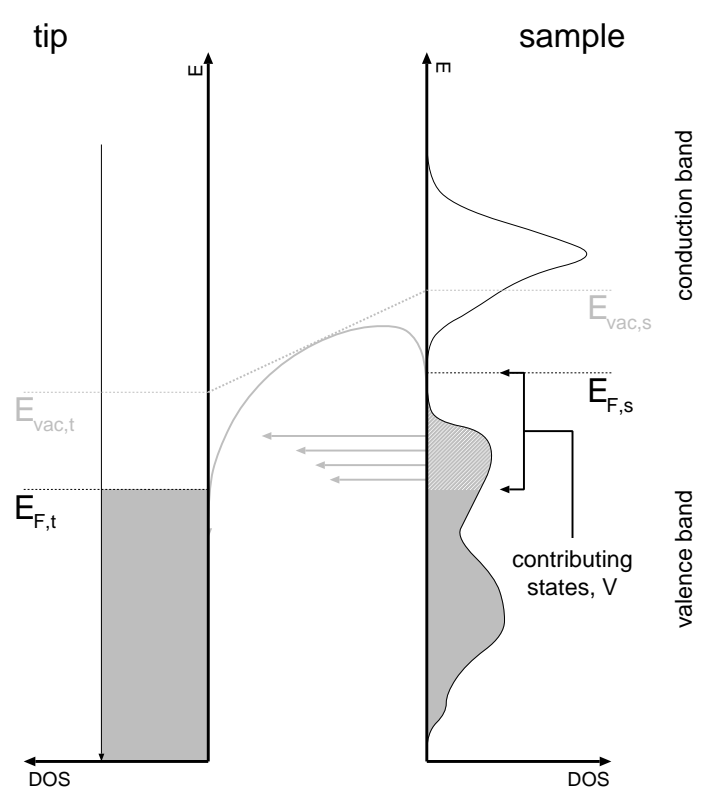

Figure 3.6: Depending on the bias voltage between tip and sample a differnt range of states contributes to the tunneling current.

Taking the minimum height along the aforementioned annulus avoids underestimation of the hillock height for hillocks in close proximity to each other. However, it comes at the cost of overestimating the hillock height by about the root mean square (RMS) roughness of the unirradiated matrix material.

The automatic detection failed for cases where the hillock height is of the order or smaller than the roughness of the matrix material (fig. 3.4). In these cases the hillocks were measured manually, possibly skewing the results as the low end of the hillock height distribution might have been hardly detectable. The results of the automatic detection runs are plotted in figure 3.5.

\subsection{Scanning tunneling microscopy}

Scanning tunneling microscopy (STM) [Bin82] is a scanning probe technique in which the probe, an atomically sharp metal tip, interacts with the sample surface through a tunneling current [Ter83, Ter85] across a controllable vacuum gap. This tunneling current $I_{t}$ depends exponentially on the gap width. $I_{t}$ decreases by about 
one order of magnitude for each angstrom the sample-tip distance increases. In constant current mode a feed-back loop couples the measured tunneling current to the tip distance control, causing the tip to approximately follow the sample topography. The tunneling current is furthermore influenced by the local density of states and thus chemical composition and structure near the sample surface.

Scanning tunneling spectroscopy (STS) allows probing of the local density of states (LDOS) of a sample. By changing the tip bias-voltage, the range of states contributing to the tunneling process can be tuned (fig. 3.6). If only elastic and non-resonant tunneling processes are considered, the tunneling current should be a monotonous function of the bias voltage. The larger the positive bias, the more occupied states in the valence band of the sample lie above the Fermi level of the tip and can source tunneling electrons. The larger the negative bias, the more unoccupied states in the conduction band of the sample lie below the Fermi level of the tip and can serve as tunneling destinations. Thus, the differential conductivity $d I / d V$ must depend on the local density of states at $E_{F}-V$. As the transmissivity of the tunneling barrier also depends on the bias voltage, one finds that a normalization with the broadly averaged total conductivity $\overline{I / V}$ yields a quantity $d I / d V /(\overline{I / V})$ which is approximately proportional to the density of states [Lan86]. Extracting an absolute scale for the LDOS requires the independent knowledge of tunnel barrier width and tunnel current. As the STM regulates the former through variation of the sample-tip distance; by measuring the latter, the LDOS distribution can only be determined qualitatively by STS. The implied approximations, a constant DOS near the Fermi level for the tip and disregard of the Fermi distribution broadening, seem reasonable on account of the tip material being a metal and the measurement being conducted at liquid helium temperatures.

The sensitivity of STM/STS is limited to a shallow surface layer. If for a given bias voltage the LDOS at or near the surface is low, electrons from deeper layers are participating in the tunneling processes, resulting in a probing of deeper atomic layers [Lon99]. In the absence of screening by quasi-free electrons, the electronic structure up to a few atomic layers deep [Joh93a] can be probed, in metallic systems the electronic screening limits the sensitivity to top-most atomic layer. For the reasons discussed above the $\mathrm{sp}^{3}$-fraction in the surface layer is expected to be below the bulk value. In crystalline materials surface reconstructions can occur, leading 


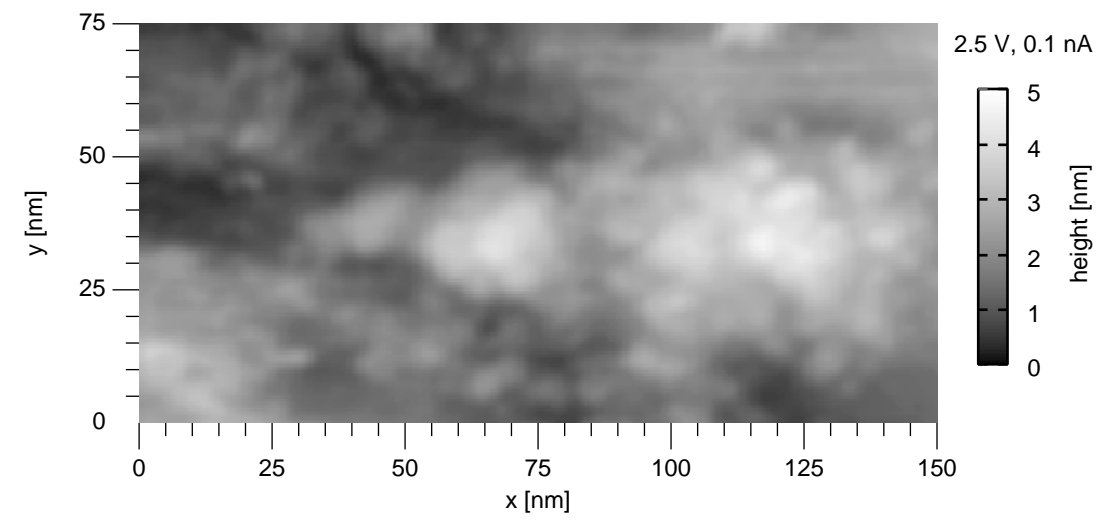

Figure 3.7: Topography image of the ta-C sample surface, taken at $2.5 \mathrm{~V}$ bias voltage and a current set-point of $0.1 \mathrm{nA}$. The measured roughness is dominated by the morphology of the underlying iron film. Locally increased conductivity, although expected to show up in the topography scans, constitutes only a small perturbation of this roughness.

to a new rearrangement of surface atoms in a two-dimensional lattice. The best known example is the $\mathrm{Si}$ (111) $7 \times 7$ reconstruction [Car81], which creates a distinct set of states [Ham86] exhibiting a metal-like LDOS. Such a reconstruction is not known for the amorphous carbon material. Thus, the measured LDOS is expected to reflect the bulk DOS with a shift to smaller band gap widths. This effect has been observed in STS measurements on hydrogen-plasma etched amorphous carbon films [IO00]. The hydrogen removes the top surface layers, while saturating dangling bonds and forming a termination layer, which preserves the $\mathrm{sp}^{3}$ hybridization of the underlying carbon atoms.

The images were acquired with the fast scan direction along the $\mathrm{x}$-axis, with topography information recorded at $2.5 \mathrm{~V}$ bias voltage and a set-point of $0.1 \mathrm{nA}$ during the trace, and measuring full IV spectra at each point during the retrace, resulting in a $I(V, x, y)$ for each measurement. This technique, called spatially resolved $I(V)$ spectroscopy, allows for direct pixelwise comparison of current maps at different bias voltages. By cycling the bias voltage at each pixel as opposed to performing multiple scans at different biases, the effects of thermal- and piezo-drift are largely avoided, and the frames for each bias match up with eachother and the topography image. 


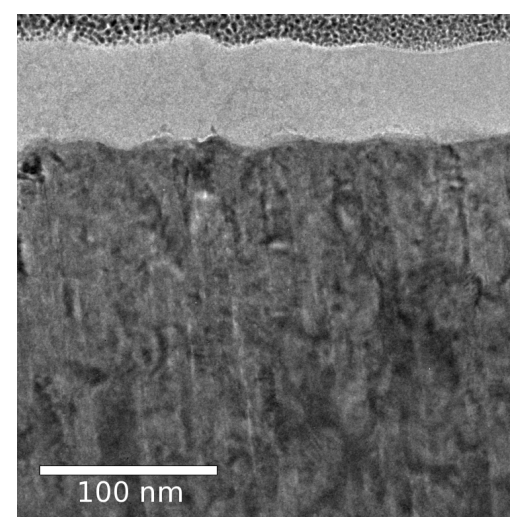

Figure 3.8: Crosssectional TEM image of a $50 \mathrm{~nm}$ ta-C film, deposited on an UHV evaporated iron film (bottom).
To increase the current resolution, the tip was automatically approached for measurements at low bias voltages. A linear tunnel barrier width reduction causes an exponential increase of the tunneling current, which then can be kept above the $5 \mathrm{pA}$ analogdigital converter (ADC) noise limit for lower bias voltages. The effect of this z-shift can be computationally removed from the spectra, yielding the expected curve for a static tip. This calculation requires knowledge of the tunnel barrier height, which can be approximated by the work-function of the tip, $4.5 \mathrm{eV}$ in the case of the tungsten tip used.

The analyzed sample was a $50 \mathrm{~nm}$ thick (fig. 3.8) ion beam deposited ta-C film on an ultra high vaccuum evaporated iron film on silicon. The carbon film follows the topography of the underlying iron film. The sample surface roughness is mainly given by the iron-carbon interface roughness. The metallized substrate was chosen to provide a highly conducting back electrode at $4 \mathrm{~K}$, the temperature the measurement was performed at. The silicon substrate, although highly boron doped to a resistance of $0.005 \Omega \mathrm{cm}$, would exhibit a considerably higher resistance.

The topography shown in fig. 3.7 is dominated by the long-range roughness of the underlying iron film, as comparisons to crosssectional TEM images (fig. 3.8) indicate. As a consequence hillocks, clearly visible on samples deposited on flat substrates, cannot readily be seen. The hillocks are not only a purely topographical feature, but due to the lowered resistivity along the underlying ion tracks a resulting tunneling current increase which would cause a tip retraction. However this retraction would be of the order of a few Angstroms only, which constitutes only a small perturbation of the visible roughness.

Two current maps taken at $0.8 \mathrm{~V}$ and $1.0 \mathrm{~V}$ bias voltage are shown in fig. 3.9 these images are sensitve to tunneling current variations at small bias voltages, and LDOS structure near the Fermi level. The film is irradiated with $10^{11} \mathrm{~cm}^{-2}$, thus, about eleven tracks are expected on the scan area of $75 \times 150 \mathrm{~nm}^{2}$. The ion track area density follows a Poisson distribution, resulting in a 30\% chance of registering less than 10 tracks in the given area. The $I(V, x, y)$ data set was filtered to remove 


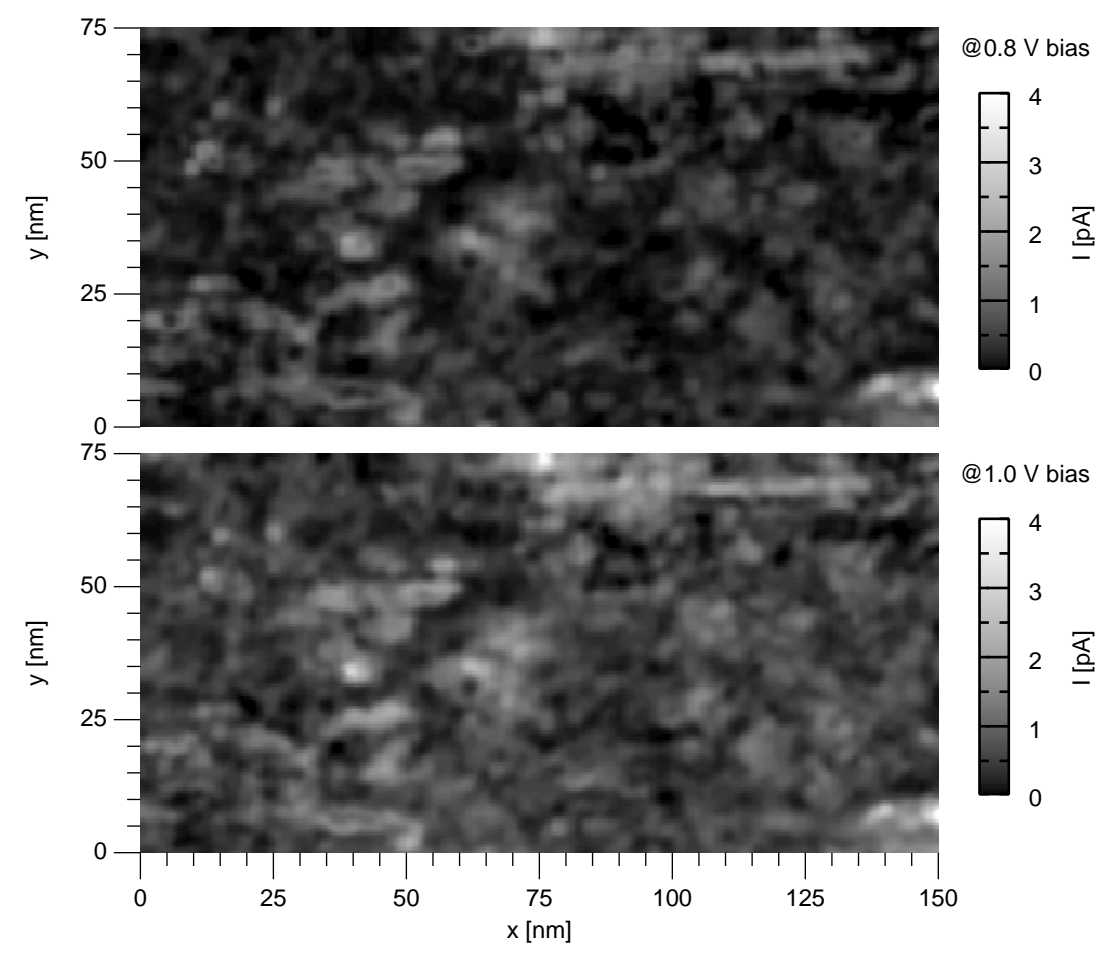

Figure 3.9: . Current maps acquired at $0.8 \mathrm{~V}$ and $1.0 \mathrm{~V}$ bias voltage. A $5 \times 5$ spatial median filter was used for each bias voltage, effectively removing datapoints with extreme current values. No tracks are clearly visible at either bias voltage individually. 


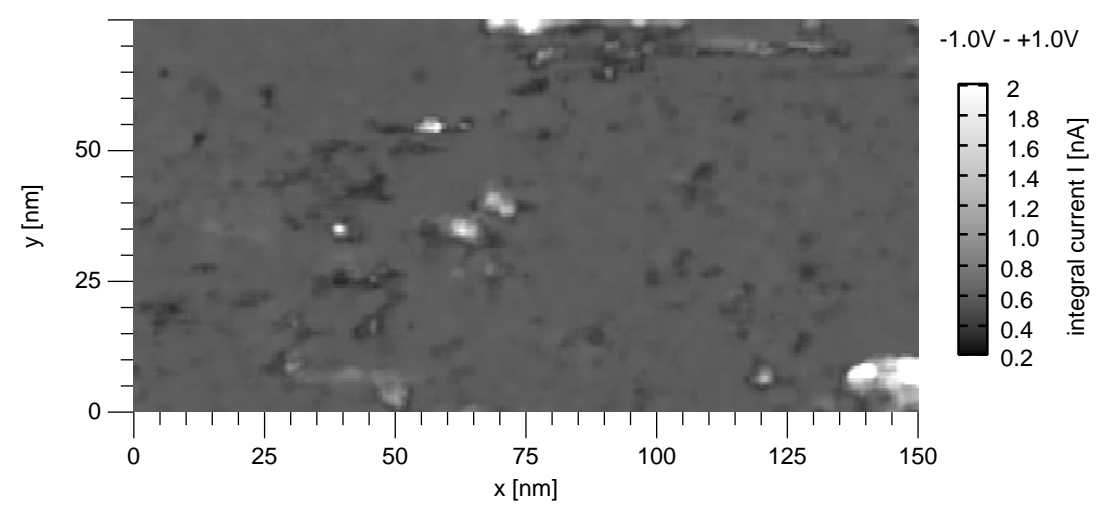

Figure 3.10: Integral current maps, computed by adding the absolute values of the current integrals from $0 \mathrm{~V}-1 \mathrm{~V}$ and $-1 \mathrm{~V}-0 \mathrm{~V}$ bias voltage. The mapped out quantity is related to the total conductivity of the sample, with bright areas corresponding to high current contributions from around the mobility gap, and dark regions corresponding to low current contributions.

spectra containing electronic glitches. A $5 \times 5$ spatial median filter was used for each bias voltage, effectively removing datapoints with extreme current values. The individual $I(V)$-spectra were smoothed by piecewise fitting of cubic splines with a width of about $0.1 \mathrm{eV}$. The bottom half of the formerly square scan area was discarded due to a distinct change in tip characteristics, possibly resulting from picking up an adsorbate.

To discern irradiated from unirradiated areas an integral current map (fig. 3.10) was computed by adding the absolute values of the current integrals from $0 \mathrm{~V}-1 \mathrm{~V}$ and $1 \mathrm{~V}-0 \mathrm{~V}$ bias voltage. The mapped out quantity is sensitive to the total LDOS in the energy interval $-1 \mathrm{~V}$ to $1 \mathrm{~V}$ around the Fermi energy, with bright areas corresponding to high, and dark regions corresponding to low current contributions. This energy range incloses the gap and tail states and is relevant for the total conductivity of the sample. The total current pulled at high bias voltages, however, is not a suitable indicator for the local conductivity, as the topography scan adjusts the tip height to a given set-point, compensating areas of high conductivity with an increased sample-tip distance. The integral current map clearly separates areas of high tail-state density from the low density background.

Out of the apparent ten areas, the $I(V)$-spectroscopy data was sampled at seven different locations (fig. 3.11) at alledged tracks (thin squares), and six unirradiated 


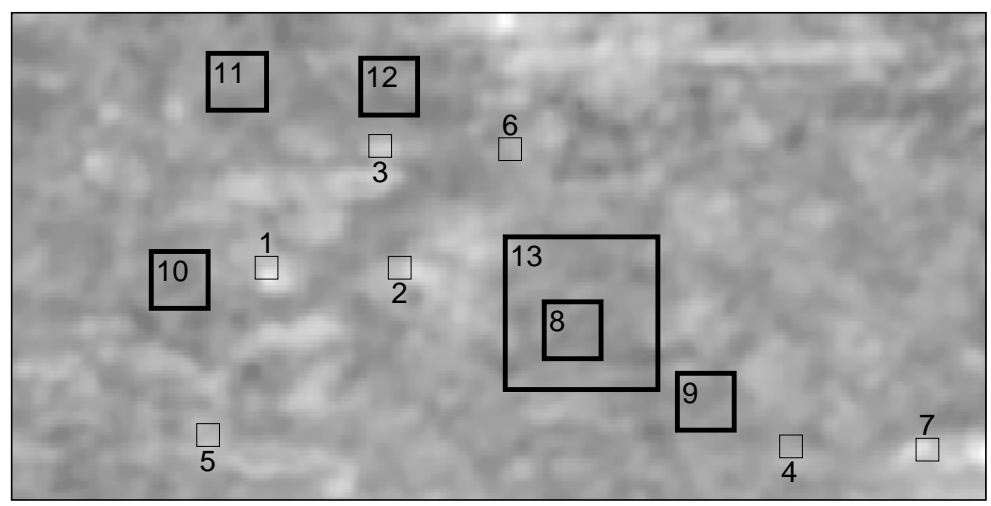

Figure 3.11: Locations of the LDOS samplings in fig. 3.12. Thin squares mark track locations, thick squares mark off-track areas. The spectra were averaged across the whole square area before computing the $d I / d V /(\overline{I / V})$ curves.

areas (thick squares). The spectra were averaged across the whole square area before computing the $d I / d V /(\overline{I / V})$ curves.

The resulting LDOS plots are shown in fig. 3.12, with the on-track curves on the left, and the curves for the unirradiated material on the right. Several gap widths are determined by tangential extrapolation of the conduction and valence band edges [Kub83]. The LDOS in the off-track areas are all consistent, exhibiting a surface mobility gap of about $1.8 \mathrm{eV}$. The on-track spectra vary between about $1.0 \mathrm{eV}$ and $0.7 \mathrm{eV}$ with one sample (4) showing a graphite-like almost closed gap.

\subsection{Current-voltage spectroscopy}

To perform transport measurements at low temperatures the samples are mounted on a CTI-Cryogenics helium cold head in a high vacuum chamber. A $50 \Omega$ heater wire wound around the cold head and attached to a Lakeshore 331 temperature controller along with two silicon diodes, on top and bottom of the sample holder were used to set and regulate temperatures ranging from $300 \mathrm{~K}$ down to $10 \mathrm{~K}$. As a sample holder a structured sapphire chip is used (fig. 3.13). Sapphire is an electrical insulator while providing excellent thermal coupling to the cold head. All leads to the sample are connected via metalized contact pads on the sample holder, minimizing thermal flux through the electrical wiring. 

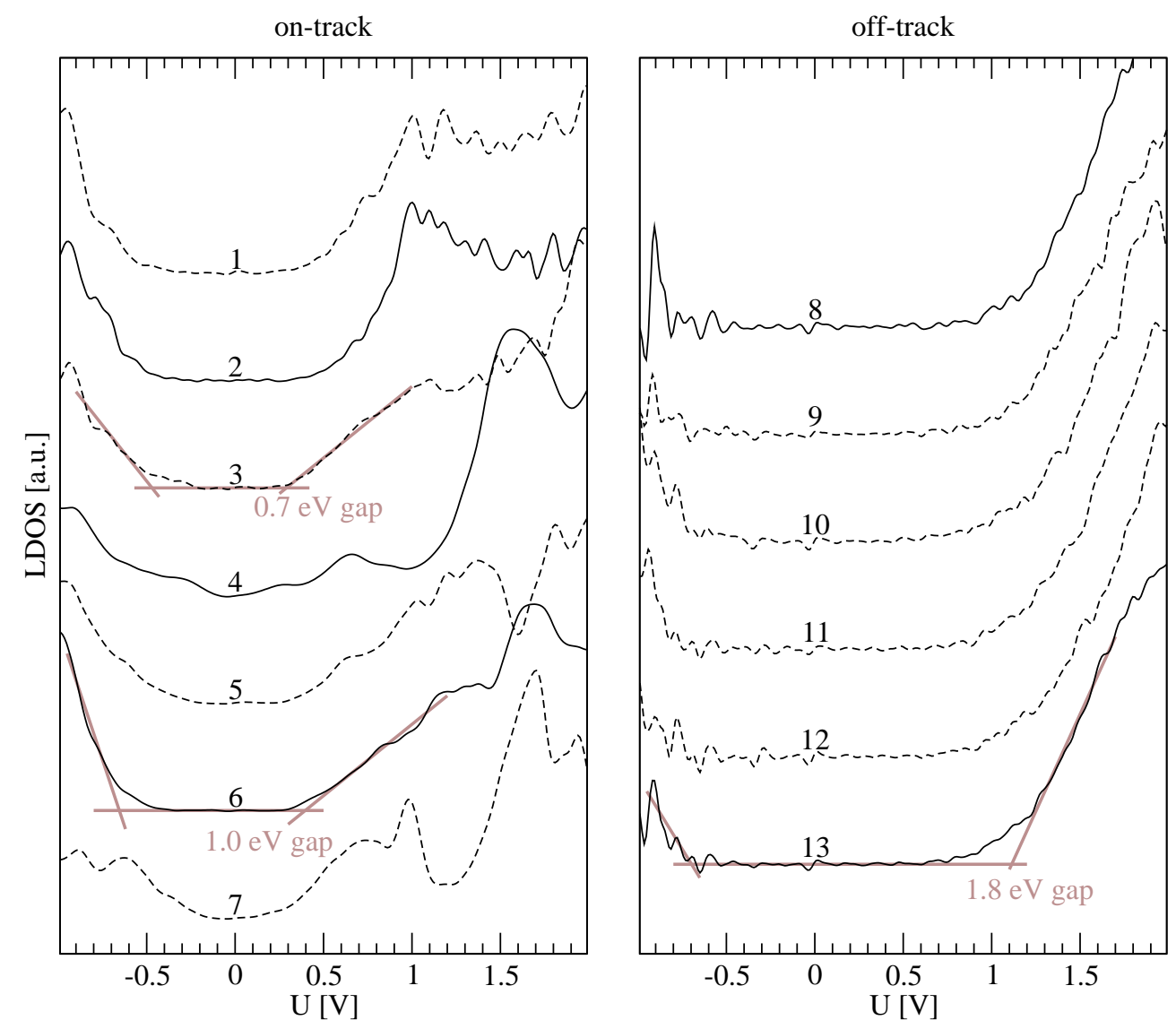

Figure 3.12: $d I / d V /(\overline{I / V})$ curves sampled at the locations shown in fig. 3.11. Several gap widths are determined by extrapolation with the tangent-method. The LDOS in the off-track areas are all consistent, exhibiting a surface mobility gap of about $1.8 \mathrm{eV}$. The track spectra vary between about $1.0 \mathrm{eV}$ and $0.7 \mathrm{eV}$ with one sample (4) showing a graphite-like almost closed gap. 


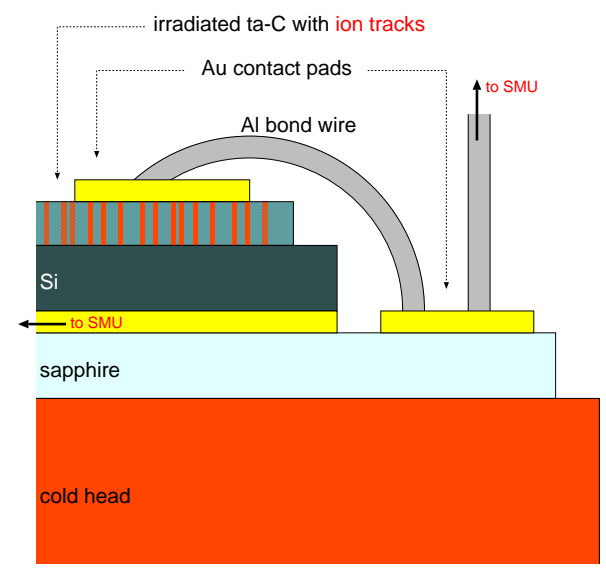

Figure 3.13: Sample setup for temperature dependent IV measurements. As a sample holder a structured sapphire chip is used. Sapphire is an electrical insulator while providing excellent thermal coupling to the cold head. All leads to the sample are connected via metalized contact pads on the sample holder, minimizing thermal flux through the electrical wiring.

A set of temperature-dependent current-voltage curves is shown in fig. 3.14. The sample is a ta-C film, deposited on a nickel (Ni) layer, irradiated with $10^{10} \mathrm{~cm}^{-2}$ ions. A set of about $10^{8}$ tracks was contacted with an evaporated $1 \mathrm{~mm}^{2}$ gold pad. No single conduction mechanism describes the measured values entirely. The $\ln I_{U}\left(T^{-\frac{1}{4}}\right)$ plots are linear for low fields and high temperatures, indicating a hopping conduction. At low temperatures and high fields higher currents are measured than expected from an extrapolated hopping behavior. The field dependence on the current increases significantly, indicating a field-dominated conduction regime. This can be explained by non equilibrium carrier distribution with an increased electronic temperature, as the electrons are acquiring energy with every hop against the applied field. A graphic extrapolation yields the carrier temperatures as a function the applied field.

A comparison of variable range hopping and Frenkel-Poole fits to an experimental IV data set is shown in fig. 3.15. The Frenkel-Poole effect describes the conduction properties only in the high temperature range, and predicts significantly lower currents at low temperatures than yielded by the measurement. Variable range hopping at moderate fields [Pol76] is in good agreement with the measured data over the entire temperature range. The inset shows the exponential hopping range depen- 


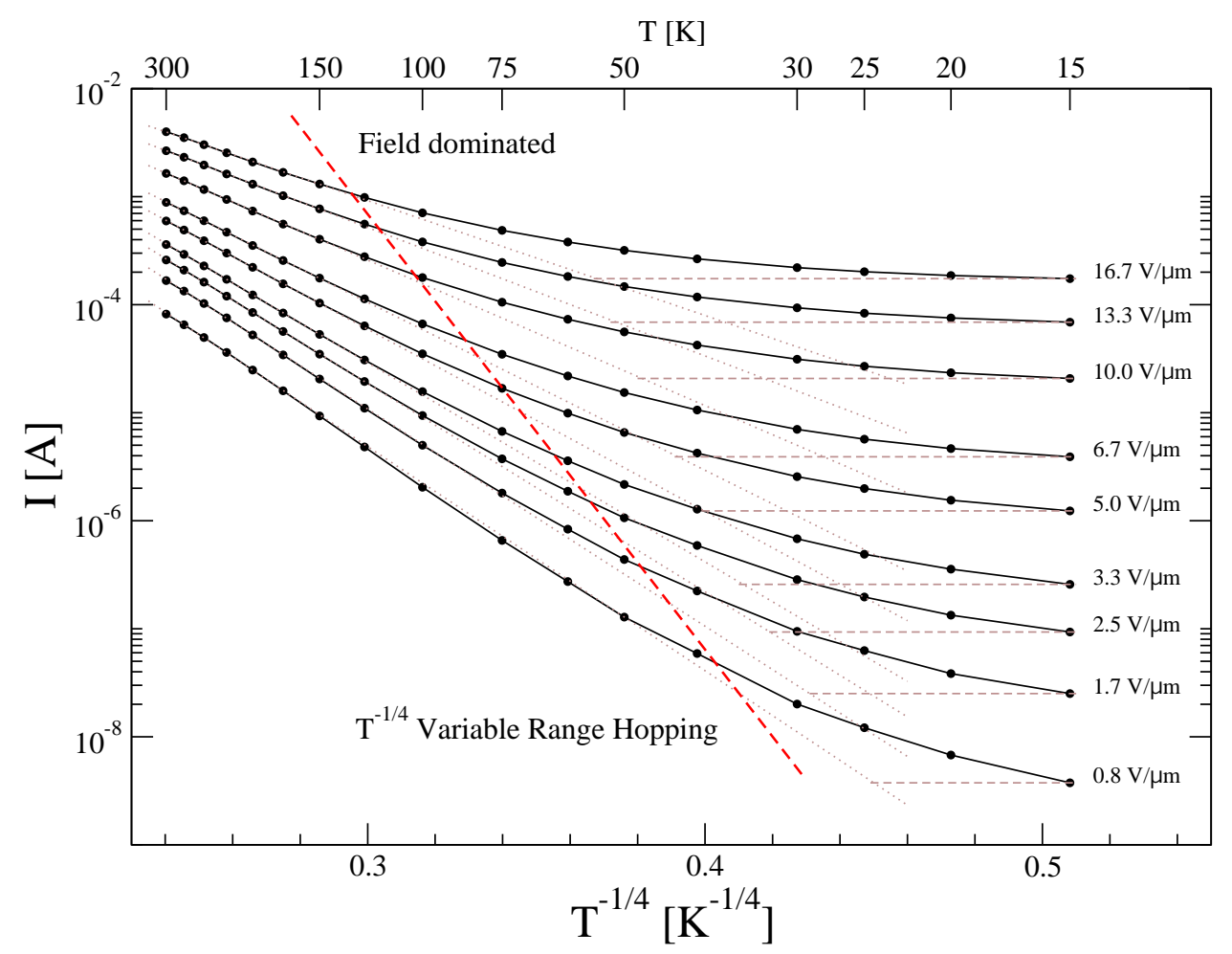

Figure 3.14: Temperature dependent current-voltage curves of a ta-C sample on Ni irradiated with $10^{10} \mathrm{~cm}^{-2}$ ions. No single conduction mechanism describes the measured values entirely. The $\ln I_{U}\left(T^{-\frac{1}{4}}\right)$ plots are linear for low fields and high temperatures, indicating a hopping conduction. At low temperatures and high fields higher currents are measured than expected from an extrapolated hopping behavior. The field dependence on the current increases significantly, indicating a field dominated conduction regime. This can be explained by non equilibrium carrier distribution with an increased electronic temperature, as the electrons are acquiring energy with every hop against the applied field. 


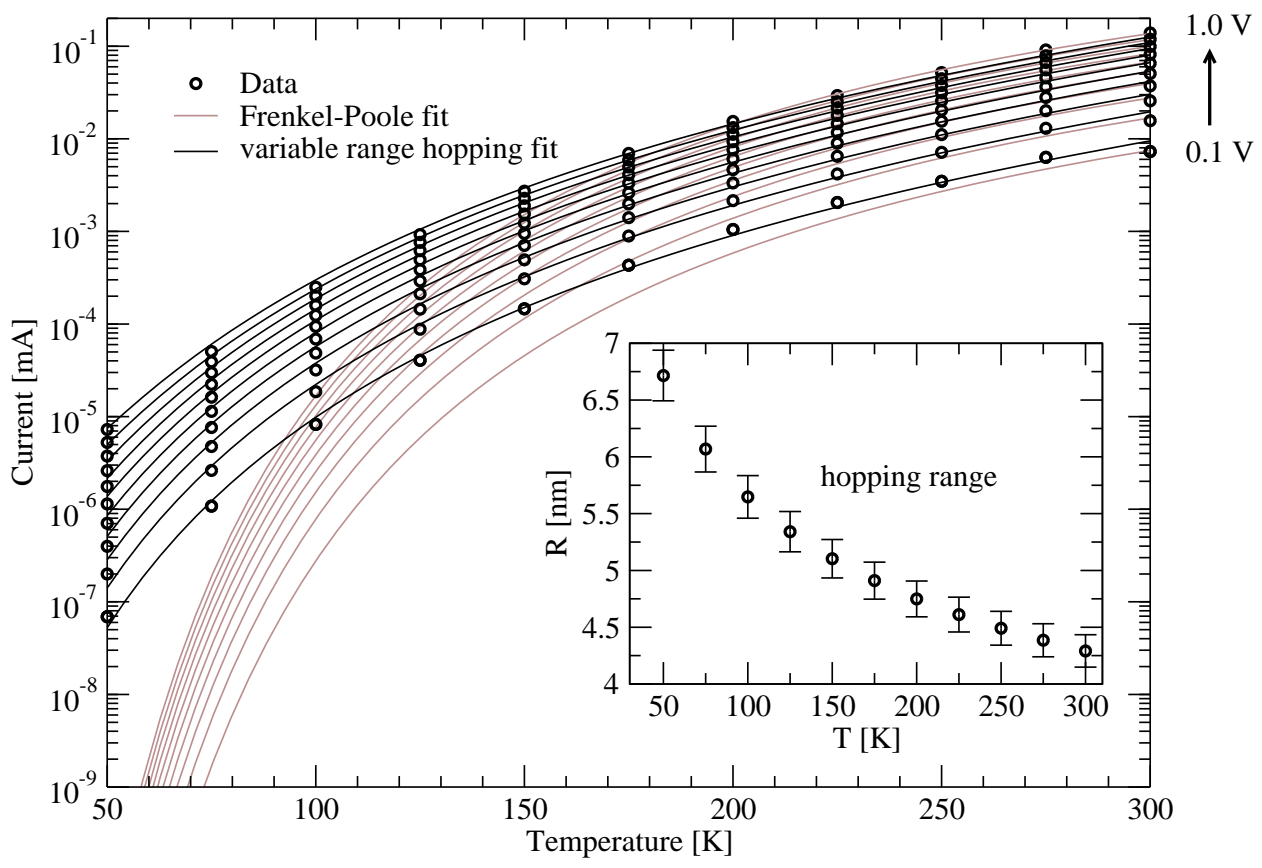

Figure 3.15: Comparison of variable range hopping and Frenkel-Poole fits to the experimental IV data set. The Frenkel-Poole effect describes the conduction properties only in the high temperature range, and predicts significantly lower currents at low temperatures than yielded by the measurement. Variable range hopping at moderate fields [Pol76] is in good agreement with the measured data over the entire temperature range. The fit parameters allow the extraction of the hopping range (shown in the inset) and the density of states contributing to the conduction. 


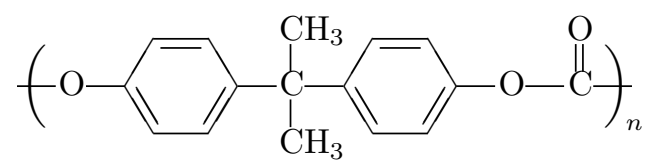

Figure 3.16: Structure formula of the polymer polycarbonate. The monomer empirical formula is $\mathrm{C}_{16} \mathrm{O}_{3} \mathrm{H}_{14}$. Makrofol, the brand used to create the spincoat-solution, consists of polymers with $n \approx 120$ [Eyn92].

cency on the temperature, extracted from the fit parameters. The density of states contributing to the conduction was determined to be of the order of $10^{19} \mathrm{~cm}^{-} 3 \mathrm{eV}^{-1}$. This value is in good agreement with electron spin resonance (ESR) measurements on ta-C films [Ama95]. A detailed discussion of further electrical measurements is presented in [Nix05].

\subsubsection{Polycarbonate masking}

Measurements on large ensembles of tracks have two key disadvantages. The electrical properties of all measured tracks are averaged, possibly over a large parameter range. If resistivity, diameter, or dielectric constant of the track material are not identical in every track, this averaging will hamper the interpretation of the involved conduction mechanisms. Secondly, by measuring through evaporated contact pads not only the tracks are contacted, but also large area fractions of pristine material in between the tracks. While increasing the irradiation fluence would reduce this fraction, track overlap becomes an issue at high fluences.

To avoid the aforementioned issues an ion-beam lithographic process has been devised to mask all unirradiated areas and allow selective deposition of metal contacts at the sites of the ion tracks. As a resist material polycarbonate (PC) was chosen, which has long been known to be a swift heavy ion beam sensitive material. The irradiation leads to chain-breaking of the polymer molecules. The fragmented chains possess an increased sensitivity to certain etchants.

The masks were prepared by spin coating a solution of PC onto the amorphous carbon samples. The solution was prepared by dissolving commercial grade PC (fig. 3.16), of the brand-name Makrofol, in dichlormethane $\left(\mathrm{CCl}_{2} \mathrm{H}_{2}\right)$, an organic 


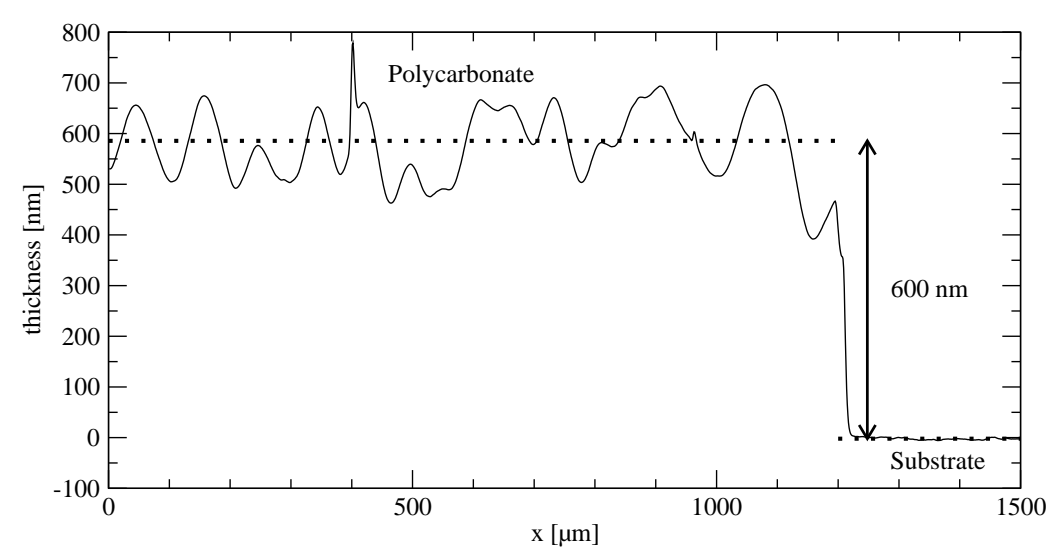

Figure 3.17: Profilometer measurements on a typical PC film, coated at $1250 \mathrm{RPM}$ with $4 \%$ solution. The film was locally removed by scratching to reveal the substrate. The observed thickness oscillations by about $200 \mathrm{~nm}$ with an approximate wavelength of $100 \mu \mathrm{m}$ can be attributed to surface tension effects prior to solidification of the PC solution.

solvent. Several coating runs were done varying concentration and rotation speed of the spincoater. Best results, leading to a uniform and reproducible thickness of about $600 \mathrm{~nm}$ (see 3.17) over large areas of our samples, were achieved with a $4 \%$ solution at a spin coater speed of 1250 RPM. The films exhibit wave-like thickness oscillations of about $200 \mathrm{~nm}$ with an approximate wavelength of $100 \mu \mathrm{m}$ which can be attributed to surface tension effects prior to solidification. This oscillation can be considered small compared to the total thickness, as long as the ratio of maximum thickness to minimum thickness is much smaller than the ratio of etch rates in the irradiated and unirradiated PC. This condition guarantees that in any case the tracks are completely etched open before the etchant dissolves the unirradiated matrix. 

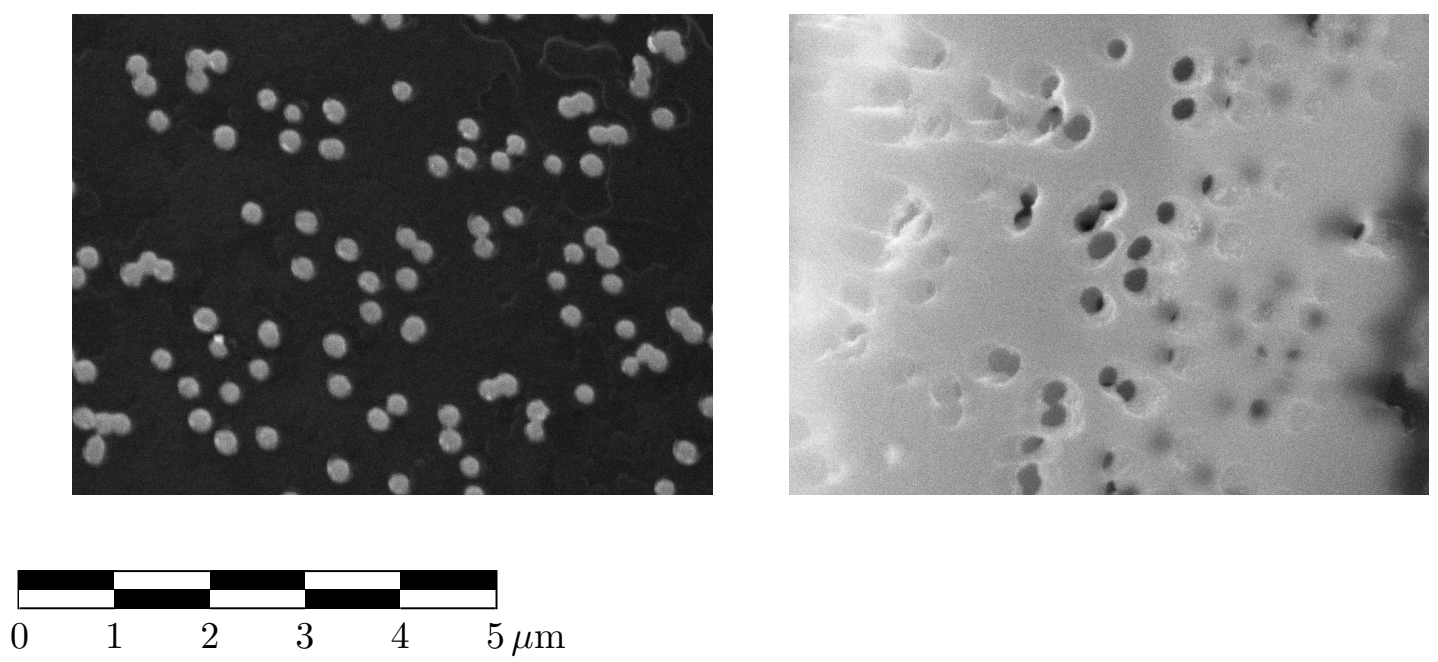

Figure 3.19: Scanning electron microscopy (SEM) images of an etched PC mask and corresponding deposited metal contacts. Left: A pattern of $200 \mathrm{~nm}$ gold dots on silicon, evaporated through an ion irradiated and etched polycarbonate mask. Right: corresponding section of the etched polycarbonate mask from below. The mask was lifted off the sample. As the thin PC film is almost transparent in the electron microscope the gold film on top and a thin gold lining inside the etch holes can be seen through the PC.

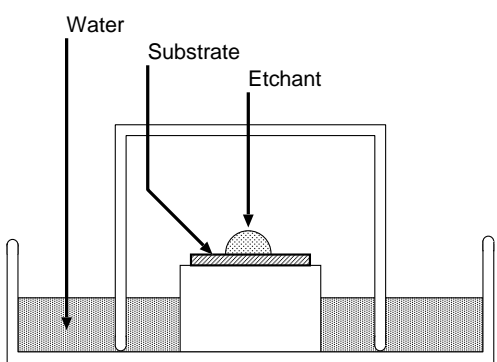

Figure 3.18: The irradiated samples were etched using a $15 \mathrm{l}$ drop of $5 \mathrm{~mol} / \mathrm{l}$ sodium hydroxide $(\mathrm{NaOH})$ solution in an air tight chamber to minimize etchant evaporation.
The irradiated samples were etched using a $5 \mathrm{~mol} / \mathrm{l}$ sodium hydroxide $(\mathrm{NaOH})$ solution. A $15 \mu$ l drop of the etchant was placed on the sample in an air-tight water-filled chamber (fig. 3.18). The water saturates the chamber with vapor, minimizing the evaporation of the solution over the etching time. After $30 \mathrm{~min}$ the etchant is removed and the sample is flushed with distilled water. In the course of the etching process the etchant is forced into the opened tracks by capillary force. As soon as the tracks are fully opened, the $\mathrm{NaOH}$ solution creeps under the film lifting it from its substrate. This effect makes the etched films unsuitable for pore-filling by electro-chemical deposition [Enc03].

Instead a dry approach was taken for the pore-filling by evaporation of a thin metal layer. The left scanning electron microscopy (SEM) image in figure 3.19 shows a pattern of $200 \mathrm{~nm}$ gold dots on a silicon substrate. On the right side the corresponding section of the etched mask is shown from below. As the thin PC 
film is almost transparent to the electron beam, the goldfilm on top and a thin gold lining inside the etch holes can be seen through the PC. A comparison of irradiation fluence, etchhole area density and deposited gold dot area density shows a good correspondence. In particular every hole in the membrane results in the deposition of a gold dot.

To create a single track contact the masked substrate has to be irradiated with precisely one swift heavy ion. This requires the detection of individual ion impacts and extremely low ion fluxes to distinguish and react on the detected impacts. The silicon substrates were mechanically thinned to a thickness of less than $1 \mu \mathrm{m}$ before the amorphous carbon films were depeosited. The thin samples allow the passage of the projectile ions, with enough remaining energy to allow for the ion detection behind the sample by means of a PIN diode. The first detection immediately triggers a beam blanker. The steps of this process are illustrated in figure 3.20.

The single ion irradiated samples were etched and coated with multiple gold contact pads, one at the site of the ion impact, and several control pads in unirradiated regions. The control measurements determine the resisitance of the polycarbonate film. With currents below of $10 \mathrm{pA}$ across the contact pad areas of approximately $1 \mathrm{~mm}^{2}$ with a bias voltage of $1 \mathrm{~V}$ applied, the leak current is well below the expected current signals from the irradiated portions. C-AFM measurements on individual ion tracks yield currents of the order of $10 \mathrm{nA}$ at $1 \mathrm{~V}$ bias voltage.

However, to this date no reproducible single ion track contact could be established. We attribute this to mechanical leeway of the PC film, either laterally displacing the gold contact from the ion track in the amorphous carbon film, a lift off due to local loss of film adhesion caused by the etching agent or insufficient pore-filling.

\subsection{Plane view TEM analysis}

While scanning force microscopy yields only direct information on the surface topography and indirect information on volume properties through electrical conductance measurements, transmission electron microscopy (TEM) can provide direct information on volume properties such as the density distribution. 

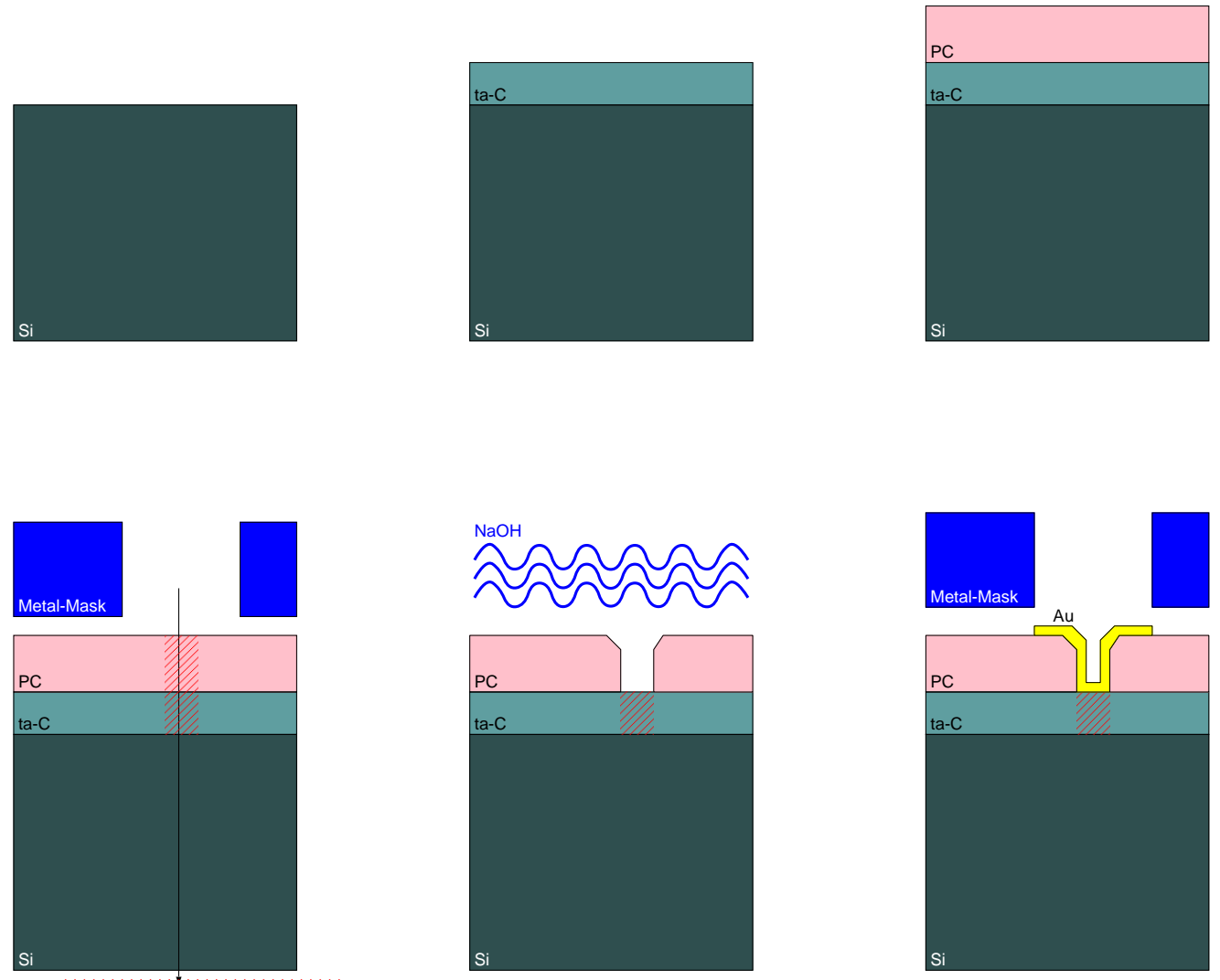

Figure 3.20: Processing steps needed to create a single track contact. From top left to bottom right: Starting with a sputter-cleaned silicon substrate, an amorphous carbon film (ta-C) is grown by ion-beam deposition. A polycarbonate film is spin coated on top of the ta- $\mathrm{C}$ and then irradiated with a single swift heavy ion. The metal mask defines the region of the ion impact. In a wet chemical etching process, utilizing a sodium hydroxide solution, the material along the ion track is removed. A small gold pad is evaporated, filling the opened channel. 

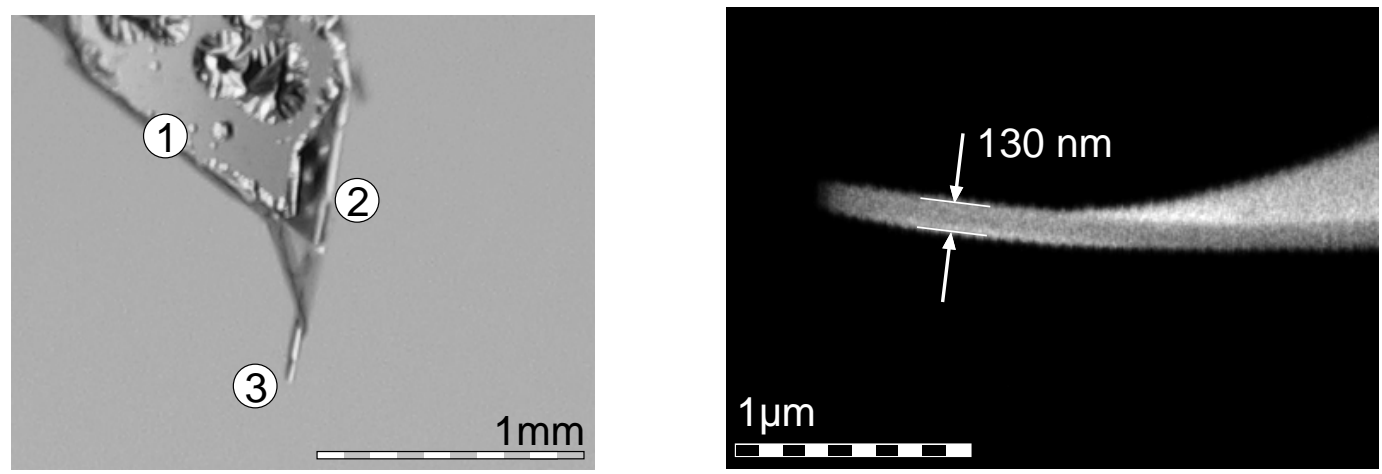

Figure 3.21: (left) Macro photo of a ta-C sample (1) after partial substrate removal through wet chemical etching. The carbon films are not attacked by the etchant and remain as free-standing foils (2). Once the supporting substrate is removed the films roll up (3) due to the compressive stress gradient introduced by the deposition process.

Figure 3.22: (right) On-edge SEM image of a free-standing carbon film. The apparent film thickness is $130 \pm 10 \mathrm{~nm}$

\subsubsection{Sample preparation}

The sample preparation for the TEM analysis in plane view geometry requires the separation of the carbon films from their silicon substrates. The good film adhesion impedes a simple physical delamination approach. Instead the silicon substrates were chemically dissolved. An etchant composed of three parts nitric acid $\left(\mathrm{HNO}_{3}\right)$ and one part hydrofluoric acid (HF) was used to dissolve the substrates. The nitric acid oxidizes the $\mathrm{Si}$ to $\mathrm{SiO}_{2}$ which in turn is dissolved by the hydrofluoric acid, resulting in the following total reaction:

$$
\mathrm{Si}+\mathrm{HNO}_{3}+6 \mathrm{HF} \rightarrow \mathrm{H}_{2} \mathrm{SiF}_{6}+\mathrm{HNO}_{2}+\mathrm{H}_{2}+\mathrm{H}_{2} \mathrm{O} .
$$

The carbon films are not attacked by this etchant and remain as free-standing foils. However, the ion beam deposition process by which the films are grown induces a compressive stress gradient perpendicular to the surface, causing the foil to roll up once the rigid substrate is removed (fig. 3.21). By masking parts of the substrate back side, a stabilizing frame of silicon can be created which suppresses the rolling up. To facilitate the transfer of foil fragments to TEM sample grids, the glue from adhesive black carbon pads used in scanning electron microscopy, was dissolved and 

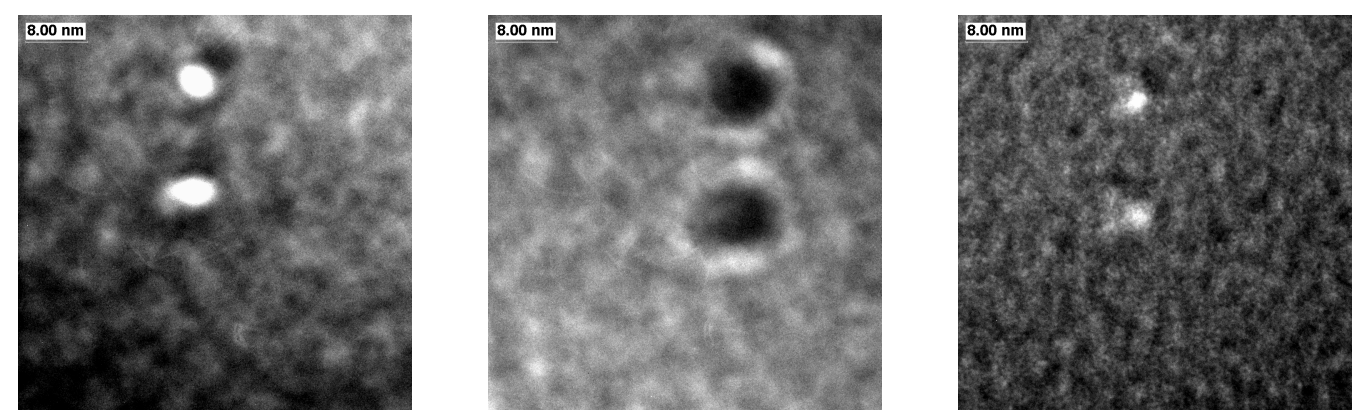

Figure 3.23: Plane view TEM images of an irradiated ta-C film with defocus settings $-7.5 \mu \mathrm{m}$, $3.6 \mu \mathrm{m}$, and $-3.4 \mu \mathrm{m}$. Area density of the point like diffraction patterns is consistent with the irradiation density of the film and suggests the identification of the patterns with the ion tracks. A sign change of the defocus length causes a contrast reversal of the track image, indicating a phase contrast. Comparisons with image simulations (fig. 2.15) allow this to be attributed to a density reduction in the track center.

diluted in acetone, and applied onto the TEM grids coating them with a minute amount of non-drying glue. The sticky TEM grids were then used to pick up tiny flakes of the prepared carbon foil, suspending them across grid openings and fixing them tightly in place with the glue coating.

The amorphous structure of the samples, the small nuclear charge $\left(Z_{C}=6\right)$, and the small density variations vindicate the weak-phase-object approximation (WPOA) for the image generation in the TEM. All images were acquired using a Philips CM200-FEG-UT operating at $200 \mathrm{keV}$ beam energy.

Plane view TEM images of an irradiated ta-C film with defocus settings $-7.5 \mu \mathrm{m}$, $3.6 \mu \mathrm{m}$, and $-3.4 \mu \mathrm{m}$ are shown in fig. 3.23. Point-like diffraction patterns with circular brightness oscillations appear on the images with equal area density as the ion fluence. A sign change of the defocus length causes a reversal of the image contrast. Cautiousness has to be execised in evaluating the diffration patterns with respect to density distribution and track diameters. Comparisons with image simulations (fig. 2.3) show the distribution of light and dark zones to be caused by a density reduction along the tracks.

Figure 3.24 shows two conical dark field (CDF) TEM micrographs of an irradiated ta-C specimen tilted by about $18^{\circ}$. The left image was taken with a $6,5 \mu \mathrm{m}$ overfocus, the right image at a $6.5 \mu \mathrm{m}$ underfocus. Defocus sign-change causes a contrast reversal, indicating a phase contrast. As the elemental composition of the film is 

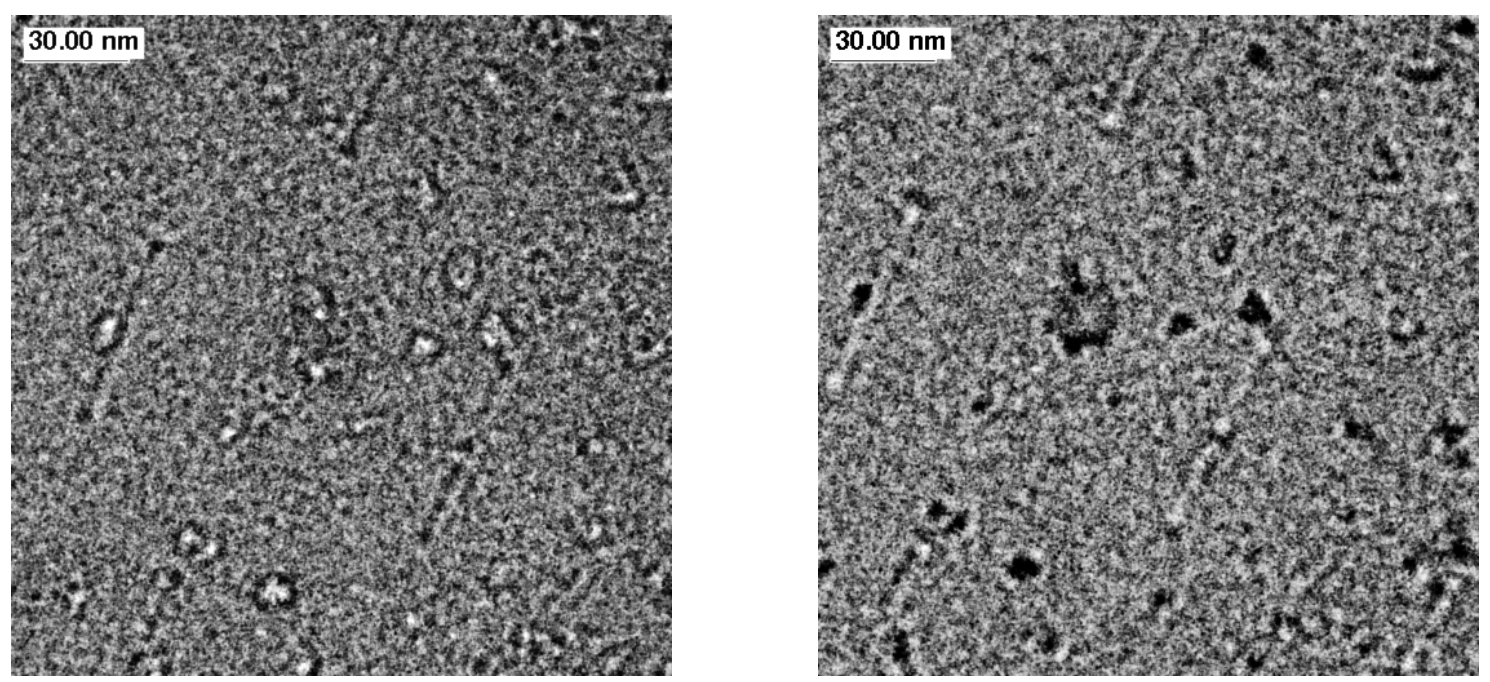

Figure 3.24: Conical dark field (CDF) image of an irradiated ta-C specimen tilted by about $18^{\circ}$. Left at $6,5 \mu \mathrm{m}$ overfocus, right at $6.5 \mu \mathrm{m}$ underfocus. Defocus sign-change causes a contrast reversal, indicating a phase contrast caused by a density reduction in a cylindrical volume.

expected to be unchanged, the phase contrast must be caused by a density reduction along a cylindrical volume. With a visible diffraction pattern length of about $50 \mathrm{~nm}$ at a viewing angle of about $18^{\circ}$ a track length of $160 \mathrm{~nm}$ can be calculated, which is consistent with cross-sectional SEM analysis of the film thickness (fig. 3.22). As expected the track extends along the entire film thickness. At the ion energies used for the irradiations studied in his thesis, the projectile ion loses less than a percent of its total energy upon film traversal, guaranteeing a virtually constant stopping power.

\subsection{Irradiated fullerene films}

As a complementary carbon based carbon thin film system fullerene $\mathrm{C}_{60}$ [Kro85, Krä90] was chosen. The films were deposited using an resistively heated Knudsen cell UHV system onto HF dipped silicon substrates at room temperature. The chamber pressure was kept at $10^{-10}$ mbar before and $10^{-8}$ mbar during deposition. 


\subsubsection{Thermal desorption}

Exposure to UV light can lead to the formation of covalent bonds between neighboring molecules [Mat93] resulting in a matrix with much stronger networking. Electron irradiation has similar effects on fullerene thin films, making them a potential resist material for electron beam lithography [Tad96].

Considering the high electronic energy deposition of swift heavy ions and their effect on amorphous carbon, $\mathrm{C}_{60}$ is likely to be a promising material for track formation by swift heavy ions. Due to the energy deposition of the ions along their trajectories a breaking of the molecular bonds and a renetworking to a low density covalently bound amorphous material is expected. Such a material would exhibit a substantially larger sublimation temperature, similar to ta-C which is expected to evaporate above $4300 \mathrm{~K}$. In the peripheral regions of the tracks the deposited energy might only be sufficient to polymerize the $\mathrm{C}_{60}$ matrix by forming covalent bonds between neighboring molecules. However, both damage mechanisms would modify the sublimation temperature and solubility locally.

Tempering of the irradiated samples would lead to thermal desorption of the pristine matrix material between the tracks, while the covalently renetworked material along the ion trajectories would be left behind. Observation of the remaining carbon pillars or piles could help determine the volume of the track damage region.

The fullerene thin films were irradiated with $1 \mathrm{GeV}$ lead $(\mathrm{Pb})$ ions and fluences of $10^{9}, 10^{10} \frac{\mathrm{ions}}{\mathrm{cm}^{2}}$, and $10^{11} \frac{\mathrm{ions}}{\mathrm{cm}^{2}}$. In a preliminary experiment the irradiated $\mathrm{C}_{60}$ films were for a few seconds dipped into 1,2,4-trichlorobenzene, which acts as a solvent for the pristine unirradiated parts. However, the results were inconclusive due to contaminations, fringes, and spot patterns possibly left behind by the evaporating solvent. This might indicate the need for longer dip times and thorough flushing of the samples.

Another sample set was irradiated and then tempered in a vacuum furnace for $10 \mathrm{~min}$ each for successive steps of $650 \mathrm{~K}, 750 \mathrm{~K}, 800 \mathrm{~K}$, and $850 \mathrm{~K}$. After each step the sample was analyzed in an SEM. After the temper step at $750 \mathrm{~K}$ holes appear to form in the film (fig. 3.25 right). Their area density is independent of the ion fluence. It seems reasonable to assume that the sublimation starts at grain boundary 

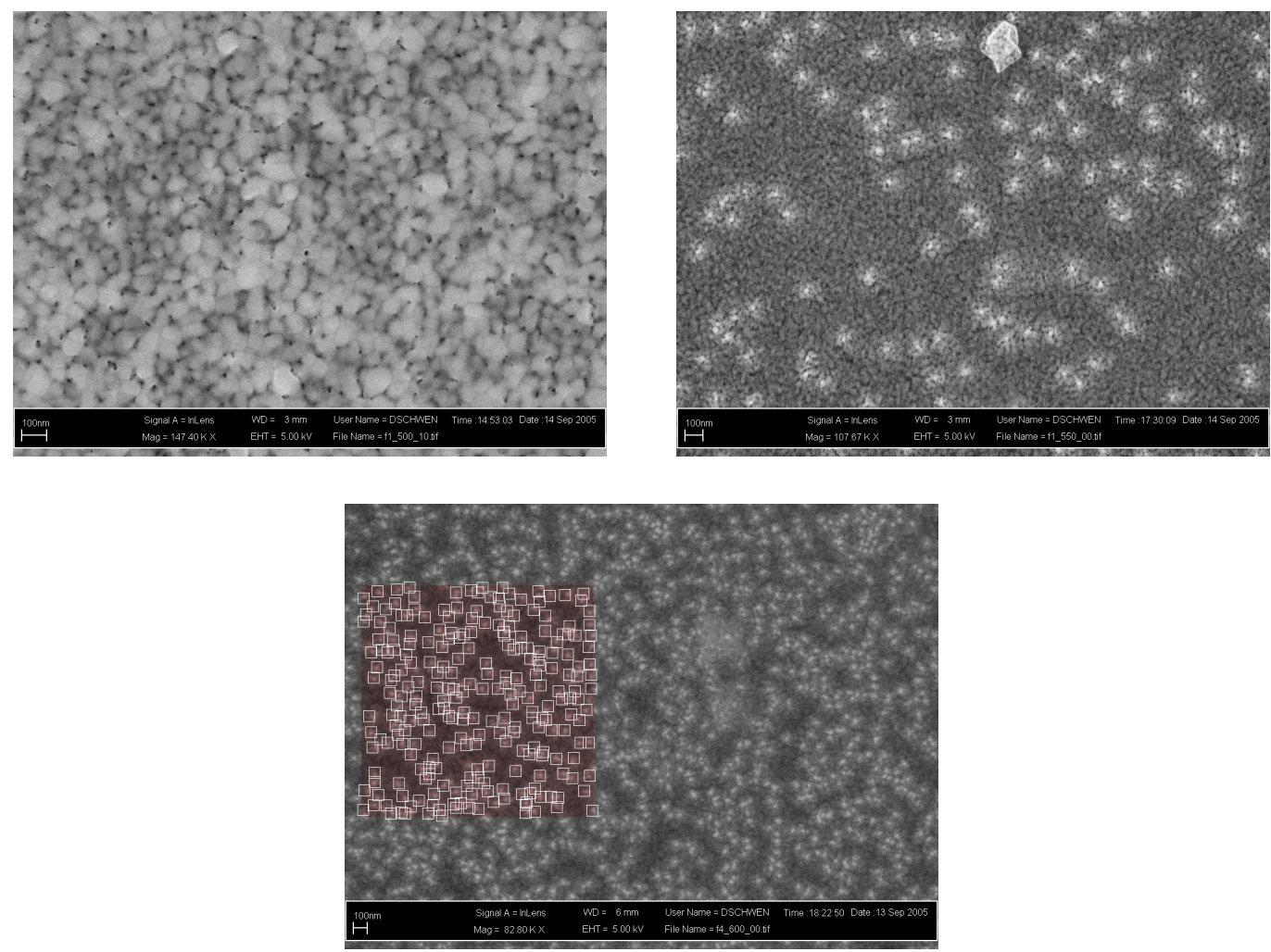

Figure 3.25: $\quad$ SEM images of an irradiated and tempered $\mathrm{C}_{60}$ film. Left: after $10 \mathrm{~min}$ at $750 \mathrm{~K}$ the columnar grains of the film are still visible. The dark $<20 \mathrm{~nm}$ spots located in the grain boundaries appear to be newly formed holes. Their area density is independent of the ion fluence. Right: after another $10 \mathrm{~min}$ at $800 \mathrm{~K}$ the matrix material disappears leaving behind small carbon piles. The area density of the piles can be estimated from the image to be about $1.4(1) \cdot 10^{9} \mathrm{~cm}^{-2}$. The irradiation fluence was a nominal $10^{9} \frac{\mathrm{ions}}{\mathrm{cm}^{2}}$. Bottom: a sample irradiated at $10^{10} \frac{\text { ions }}{\mathrm{cm}^{2}}$ after 10 min tempering at $850 \mathrm{~K}$ the $\mathrm{C}_{60}$ matrix is evaporated. The SEM image shows remaining and carbon piles with an area density of $0.9(1) \cdot 10^{10} \mathrm{~cm}^{-2}$. This coincides with the irradiation fluence. 
intersections where the $\mathrm{C}_{60}$ molecules are less densely packed, undercoordinated, and therefore weakly bound.

After further annealing at $800 \mathrm{~K}$ the appearance of the sample in the SEM changes drastically. The film gets sublimated with tiny carbon piles remaining (fig. 3.25 left). The area density of piles on the sample surface estimated from the image is about $1.4(1) \cdot 10^{9} \mathrm{~cm}^{-2}$. This corresponds well with the irradiation fluence which was determined to be $10^{9} \frac{\text { ions }}{\mathrm{cm}^{2}}$. It should be noted that the precision of the fluence measurement depends on the precision of the flux measurement and the irradiation time. Comparatively low fluences of $10^{9} \frac{\text { ions }}{\mathrm{cm}^{2}}$ tend to be uncertain to a few $10 \%$ [Tra06].

The fluence dependence of the carbon piles is confirmed by samples with different irradiation fluences. A sample irradiated at $10^{10} \frac{\text { ions }}{\mathrm{cm}^{2}}$ after $10 \mathrm{~min}$ tempering at $850 \mathrm{~K}$ the $\mathrm{C}_{60}$ is shown in the bottom viewgraph of fig. 3.25. Again the matrix is evaporated and the SEM image shows remaining carbon piles with an area density of $0.9(1) \cdot 10^{10} \mathrm{~cm}^{-2}$. This is again in good agreement with the irradiation fluence.

\subsubsection{Raman measurements}

The $\mathrm{C}_{60}$ molecule is comprised of 60 identical atom sites arranged in the shape of a regular truncated icosahedron. Due to this high structural symmetry theoretical calculations of Raman active modes were very successful, leading to a good understanding of the fullerene Raman spectrum [Dre96]. Particular modes are very sensitive on the existence of pristine $\mathrm{C}_{60}$ molecules. Comparison of signal intensities may provide information on the volume fraction of ion beam modified material.

The measurements utilize the $514.5 \mathrm{~nm}$ argon (Ar) laser line, which may provide enough energy for photo-chemical modifications such as bond breaking and renetworking. Thus reliable results require the minimization of intensities and exposure times. Repeated measurements on one sample spot document the decay of the $\mathrm{C}_{60}$ signals due to laser modification.

Only intermolecular modes were taken into consideration, as the intramolecular modes appear at wavenumbers below $60 \mathrm{~cm}^{-1}$ and were not accessible with the available Raman setup. The dominant line in the fullerene Raman spectrum is the $A_{g}(2)$ pentagonal pinching mode at $1468 \mathrm{~cm}^{-1}$, a tangential breathing of pentago- 


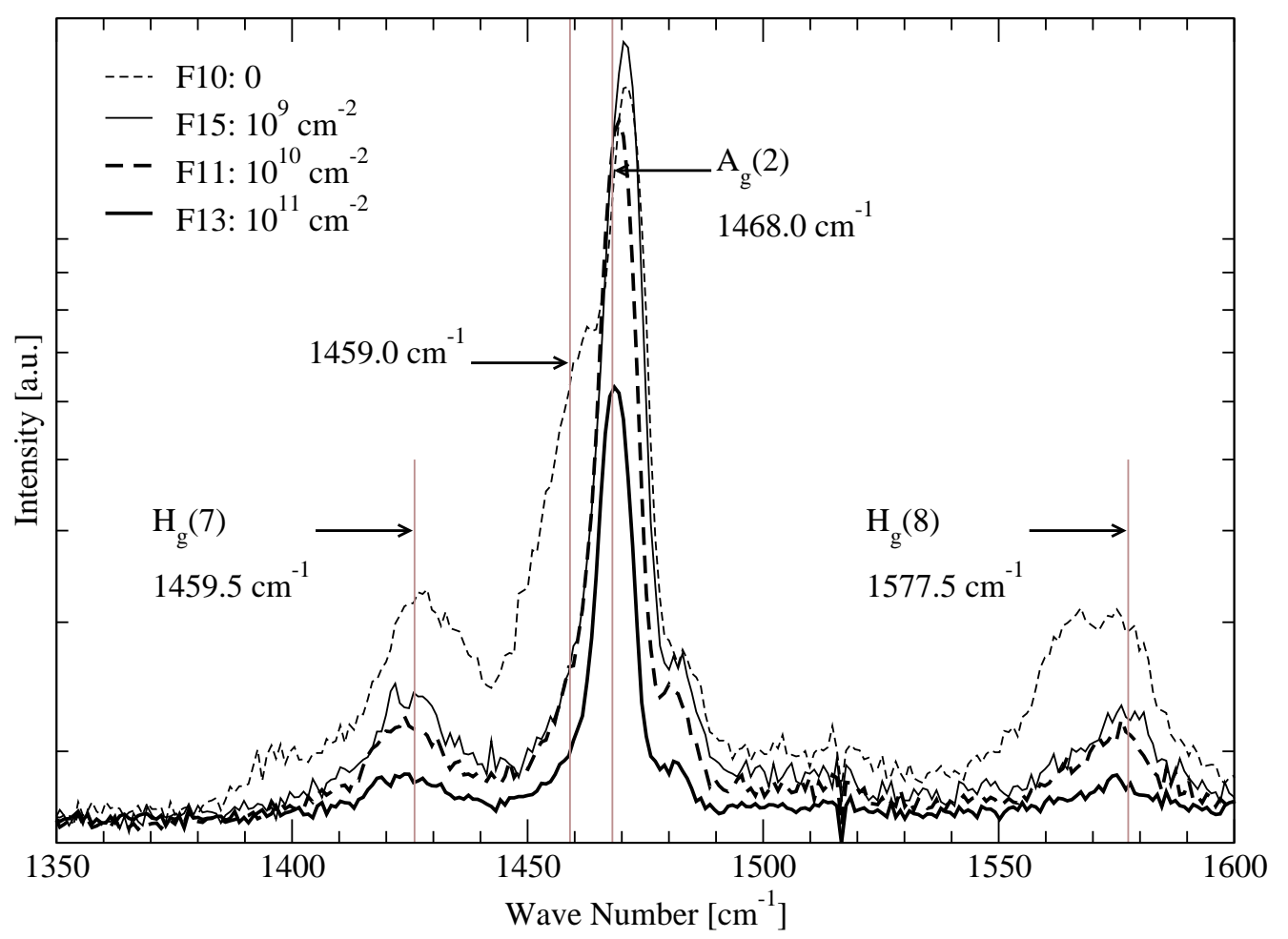

Figure 3.26: Raman signal of unirradiated and irradiated $\mathrm{C}_{6} 0$ films. The spectrum is dominated by the $A_{g}(2)$ pentagonal pinching mode peak at $1468 \mathrm{~cm}^{-1}$. This line occurs only in the presence of fully intact $\mathrm{C}_{60}$ molecules [Dre96]. Up to $10^{10} \frac{\text { ions }}{\mathrm{cm}^{3}}$ the $A_{g}(2)$ line intensity stays constant. The $50 \%$ decrease of the $A_{g}(2)$ line intensity at $10^{11} \frac{\text { ions }}{\mathrm{cm}^{2}}$ indicates substantial damage of the $\mathrm{C}_{60}$ matrix. The unirradiated sample shows a pronounced broad peak at $1459 \mathrm{~cm}^{-1}$, which is a perturbed $A_{g}(2)$ line from scattering at cross linked molecules due to photo-transformation [Mat93].

nal atom configurations. This line occurs only in the presence of fully intact $\mathrm{C}_{60}$ molecules [Dre96]. Cross linking of fullerene molecules through covalent bonding leads to a perturbation of the $A_{g}(2)$ manifesting as a broad peak centered around $1459 \mathrm{~cm}^{-1}$.

Raman spectra acquired for unirradiated and irradiated fullerene thin film samples are plotted in figure 3.26. Up to $10^{10} \frac{\text { ions }}{\mathrm{cm}^{2}}$ the $A_{g}(2)$ line intensity stays constant. The sample irradiated with $10^{11} \frac{\text { ions }}{\mathrm{cm}^{2}}$ shows an intensity decrease by a factor of two. Assuming a linear dependence of signal intensity and unmodified film volume, the volume of material modified by a single ion impact can be calculated as $(2$. $\left.10^{11} \frac{\mathrm{ions}}{\mathrm{cm}^{2}}\right)^{-1} \approx 500 \frac{\mathrm{nm}^{2}}{\mathrm{ion}}$, yielding a track radius of about $13 \mathrm{~nm}$. This radius is large 

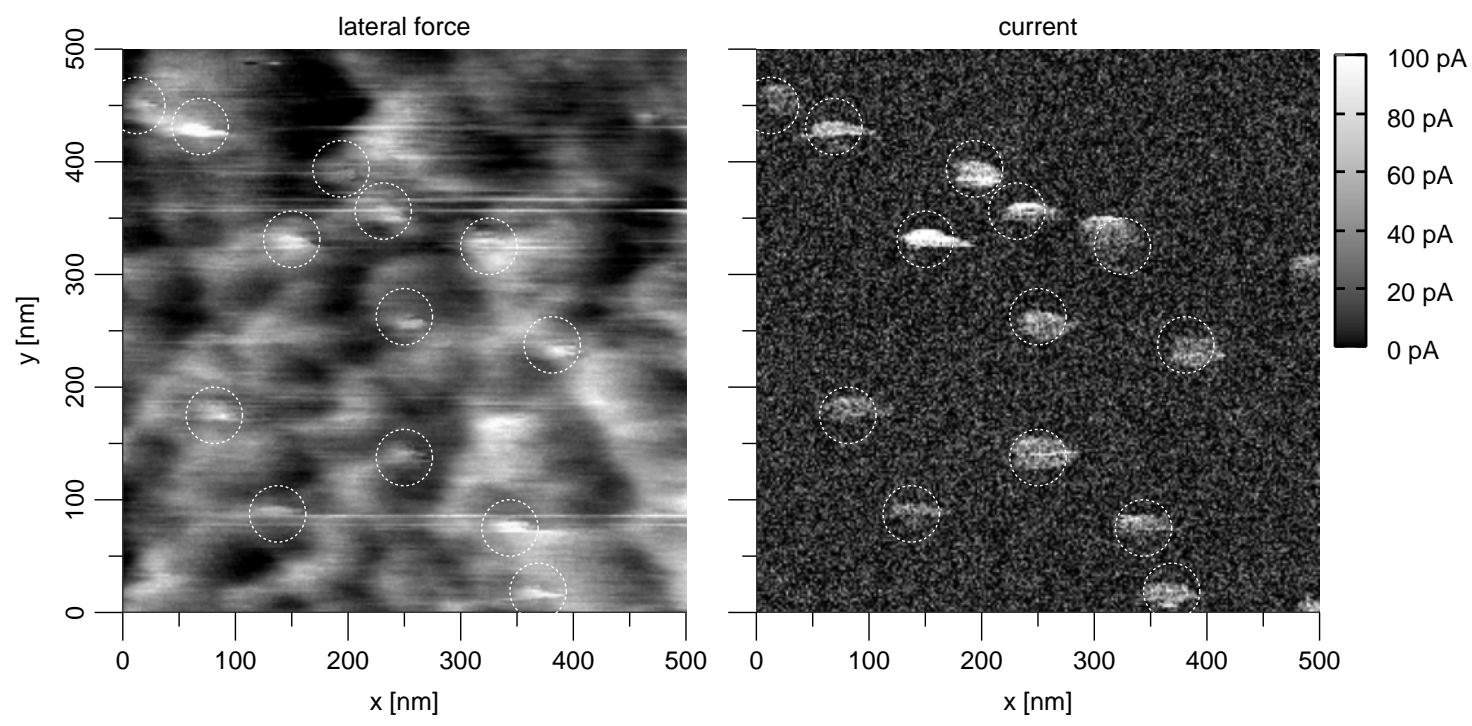

Figure 3.27: Comparison of lateral force and C-AFM images of a $\mathrm{C}_{60}$ film irradiated with $10^{10} \mathrm{~cm}^{-2} 1 \mathrm{GeV}$ lead ions. Bright areas correspond to lateral forces coefficients and high conductivity respectively. The visible track area density is about $50 \%$ of the nominal fluence. FWHM of the tracks in the lateral force image is about $8 \mathrm{~nm}$ in the slow scanning direction and $25 \mathrm{~nm}$ in the fast scanning direction.

compared to experimentaly determined radii in amorphous carbon through AFM and TEM measurements, and the radii used in the computer simulations of track formation in amorphous carbon.

The unirradiated sample shows a pronounced broad peak at $1459 \mathrm{~cm}^{-1}$, indicating cross linking due to photo-transformation [Mat93] in the presence of oxygen and high intensity laser irradiation [Duc91]. This can be attributed to the first series of measurements being performed on this sample at high energy densities, which were reduced to the lowest possible value while still retaining a reasonable signal to noise ratio.

\subsubsection{AFM measurements}

AFM measurements were performed on the fullerene films directly after irradiation (fig. 3.27). As any atomic rearrangement with formation of an amorphous carbon material will only lead to a density increase the appearance of surface hillocks is not expected. 
The lateral force is depending on the friction coefficient and the slope of the sample in the fast scanning direction. If the long range lateral force features are taken to be caused by the sample surface topography then the remaining spot-like areas of high lateral force can be identified as high friction sites.

An ideal fullerene film at room temperature behaves like stack of ball bearings. At room temperature each $\mathrm{C}_{60}$ can freely rotate around its center of mass. Furthermore the saturated $\mathrm{C}_{60}$ surface is expected to have similar adhesion properties as a graphene sheet. Renetworking along the heated ion track creates surface patches of amorphous material with a higher fiction coefficient.

The visible track area density is about $50 \%$ of the nominal fluence. The full width at half maximum (FWHM) of the tracks in the lateral force image (fig. 3.27 left) is about $8 \mathrm{~nm}$ in the slow scanning direction and $25 \mathrm{~nm}$ in the fast scanning direction. This anisotropy is visible in the current image (fig. 3.27 right) as well, and is likely to be the result of the convolution of sample and tip image. The shape of the metalized contact-mode tips is changed over the course of the measurement due to abrasion, which explains the correlation of anisotropy and scan direction. With the assumption of a cylindrical track and thus circular surface features the upper bound of the track diameter can be set at about $8 \mathrm{~nm}$, a value consistent with observations on amorphous carbon. 


\section{Chapter 4}

\section{Discussion and conclusions}

Aim of this discussion is to determine the relation of the electronic energy loss of the projectile ions and the effective energy loss deposited into the lattice within the track volume. The treatment of the ion track formation process as a thermal spike is motivated by the short timescales of the electronic energy transfer to the lattice (fig. 1.7). Effective energy losses for beginning track and hillock formation set a window in which the experiments must be positioned. Electronic energy losses in the experiments range from about $12 \mathrm{keV} / \mathrm{nm}$ to $70 \mathrm{keV} / \mathrm{nm}$, and all experiments show track formation. From the simulations the lower effective energy limit for the track formation in amorphous carbon can be put at $4.0 \mathrm{keV} / \mathrm{nm}$, the minimum energy needed to reach the saturation limit of the density reduction in the amorphous samples.

Both graphite and diamond simulation exhibit the formation of continuous ion tracks in the simulation runs at effective energy thresholds of $4.4 \mathrm{KeV} / \mathrm{nm}$ and $7.5 \mathrm{keV} / \mathrm{nm}$ respectively. The electronic energy losses in all three carbon materials are comparable and only scale with the material density. However, no continuous track formation has been reported experimentally in either material. It could be argued that diamond, as the highest density carbon phase at standard conditions, cannot accommodate the densification needed in the track periphery to compensate for the reduced density in the track core. The excess material from the full graphitization of a cylindrical volume with a radius of $5 \mathrm{~nm}$ would, if distributed across a cylindrical volume with a radius of $25 \mathrm{~nm}$, only lead to a density increase of 1.5\%. As compressibility calculations (fig. 2.3 bottom) based of the Brenner poten- 
tial confirm, such a density increase would lead to a compressive stress increase to about $3 \mathrm{GPa}$, a pressure which can easily be sustained by the amorphous track material. Even if the underestimated bulkmodulus of diamond in the Brenner potential is considered, the resulting compressive stress is still below the intrinsic stress of ion beam deposited amorphous carbon. The simulations confirm the existence of compression shockwaves with ranges well in excess of $25 \mathrm{~nm}$, and simulated density profiles support a long range compression. The incompressibility argument does not apply to the low density material graphite. In fact discontinuous damage trails have been observed in highly oriented pyrolytic graphite (HOPG) [Liu01], but only energy losses above $18 \mathrm{keV} / \mathrm{nm}$ result in a $100 \%$ yield. Furthermore radial extent is much lower than observed in the simulations, and below the surface the tracks manifest only in an interrupted chain of defects, unlike predicted in the simulation results.

The absence of continuous ion tracks in graphite and diamond is, therefore, largely attributed to the energy conversion process from the electronic system to the lattice. This energy conversion has to be less efficient in the two crystalline materials than in the amorphous carbon. The high energy electrons, created by the passage of the swift heavy ion, travel ballistically in both graphite and diamond due the low concentration of scattering centers, leading to an energy dispersion. This reduces the energy density transferred to the lattice. The mean free path in amorphous carbon on the other hand is expected to be much shorter of the order of interatomic distances.

The timescales explored in the presented MD simulations are limited to the order of $10 \mathrm{ps}$. The surrounding crystalline matrix could lead to a template guided recrystallization of the track material progressing from the track-matrix interface to the core. Kinetic Monte Carlo simulations [Lan00] or temperature accelerated dynamics (TAD) [Ube04] could be used to investigate long timescale behaviour.

For amorphous carbon MD simulations yield tracks with diameters around $6 \mathrm{~nm}$, while TEM investigations (fig. 3.23) with the help of image simulations (fig. 2.15) put the track diameter at about $10 \mathrm{~nm}$. Assuming that the energy density determines the degree of amorphization in the track core, and with the initially excited radius approximately determining the resulting track size, a new set of simulations can be proposed. In this set the initially excited radius would have to be changed 


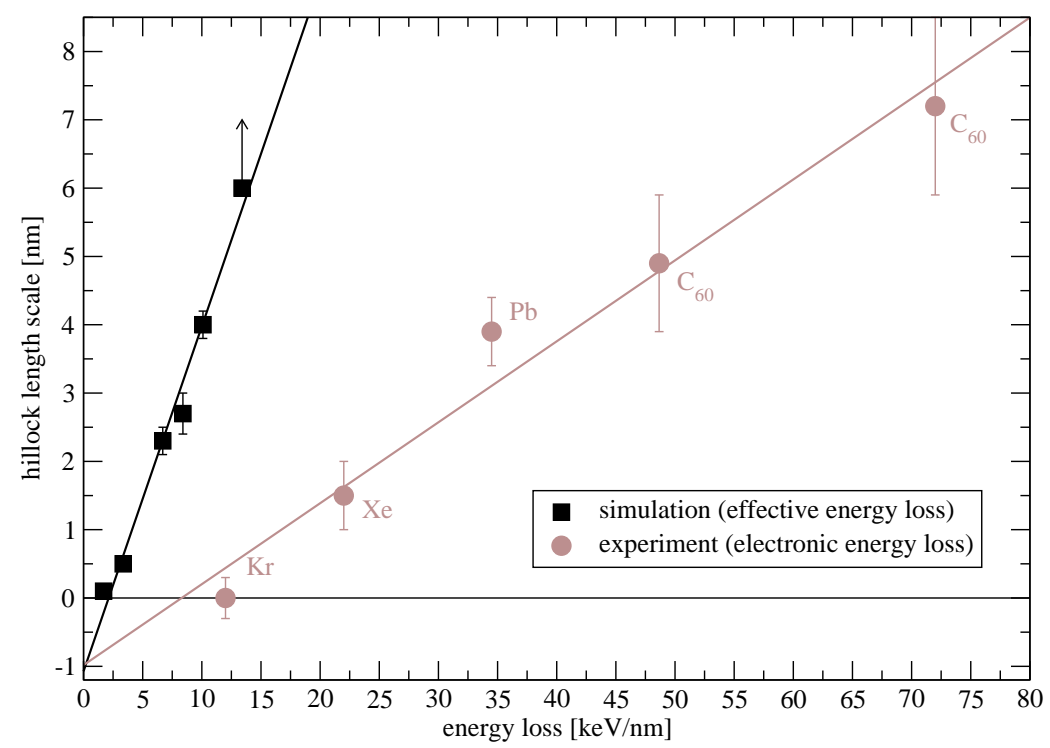

Figure 4.1: Comparison of simulated and experimental hillock heights with linear fits according to 2.1.5. The energy loss for the simulated data points is the effective $d E / d x$ provided as local lattice heating, while the experimental hillock heights are plotted as a function of the electronic energy loss. Both simulation and experiment agree reasonably well with a linear dependence hillock height on energy loss. Furthermore both data sets exhibit a similar energy threshold behavior for the hillock formation, and matching y-axis intersections. This strongly suggests a linear scaling factor in energy to relate the electronic energy loss of the projectile atom to the effective energy available for atomic rearrangement.

from $3 \mathrm{~nm}$ to $5 \mathrm{~nm}$ while keeping the energy density constant, resulting in an increase of the effective stopping by a factor of about 2.5, yielding a stopping power range from $3.4 \mathrm{keV} / \mathrm{nm}$ to $33.5 \mathrm{keV} / \mathrm{nm}$. These values are much closer to the actual experimental values used.

Figure 4.1 shows a comparison of simulated and experimental hillock heights with linear fits according to 2.1.5. The energy loss for the simulated data points is the effective $d E_{\text {eff }} / d x$ provided as local lattice heating, while the experimental hillock heights are plotted as a function of the electronic energy loss $d E_{\text {el }} / d x$. Both simulation and experiment agree reasonably well with a linear dependence hillock height on energy loss. Furthermore both data sets exhibit a similar energy threshold behavior for the hillock formation, and matching y-axis intersections. This strongly suggests a linear scaling in energy to relate experimental to simulated data sets. 
Thus, determining the ratio of the electronic energy loss of the projectile atom to the effective energy available for atomic rearrangement

$$
\frac{d E_{\mathrm{eff}}}{d x}=\gamma^{-1} \cdot \frac{d E_{\mathrm{el}}}{d x}
$$

For the data sets depicted in fig. $4.1 \gamma \approx 4.6$ can be calculated.

The plane view TEM images (fig. 3.23) of the irradiated carbon film are in good agreement with the simulated images with a track diameter of $10 \mathrm{~nm}$ (fig. 2.15). However, the molecular dynamic simulations yield track diameters of about $6 \mathrm{~nm}$. The high resolution image simulations based on the MD data sets exhibit considerably smaller diffraction patterns than the experimental TEM micrographs. A comparison with the hillock diameter behavior indicates that the track diameter is mainly governed by the size of the initially excited region.

A linear up-scaling of the radius of the initially excited thermal spike, while keeping the energy given to each atom inside the spike constant, would lead to a quadratic increase in deposited energy. At the same time the footprint of the ensuing hillock would increase quadratically. Assuming a linear dependence of deposited energy and hillock volume this would lead to the hillock height staying constant. Simulations to confirm the identical scaling behavior of deposited energy and hillock footprint with increased thermal spike radius are still pending. For the data sets presented here, an up-scaling from $3 \mathrm{~nm}$ radius used in the simulation runs to a $5 \mathrm{~nm}$ radius as observed in the TEM micrographs, would result in a rescaling of the effective energy loss scale by a factor of $\left(\frac{5}{3}\right)^{2} \approx 2.8$. This results in a conversion efficiency for electronic energy loss to effective energy available for atomic rearrangement of about $60 \%$, with the remaining $40 \%$ going into delta or convoy electrons and radiative recombinations.

The results of the STS measurements clearly show a change in the density of states in selected areas. Two distinct sets of mobility gap widths were identified on the irradiated sample. Large areas exhibited gap widths of about $2 \mathrm{eV}$. These areas were taken to be the unirradiated matrix material. Furthermore a number of spot-like areas exhibiting gap widths below $1 \mathrm{eV}$ were detected. This gap width reduction is in agreement with the hybridization change from $\mathrm{sp}^{3}$ to $\mathrm{sp}^{2}$ along the ion trajectories. As the STS measurements only probe a shallow surface layer, a bias to higher $\mathrm{sp}^{2}$ contents and, thus, narrower mobility gaps is to be expected. 
The DFT calculations on MD sub-samples reflect this trend in gap-widths, but are exhibiting a non-zero density of states in the gap region. This high DOS is assumed to be either an artifact of the empirical potential MD, or the tight-binding structure relaxation needed to force periodic boundary conditions on the subsamples taken from much larger MD data sets. The REBO empirical potential used in the MD simulations is fitted to bulk parameters and hydro-carbon molecules. It yields good results for the systems used in this fitting process and structurally similar systems. Cautiousness has to be exercised in when applying these potentials to new applications. The known shortcoming of the low interaction range has direct influence on the local microstructure. While the cut-off radius increase proposed by Jäger and Albe restores expected bulk properties of an average $\mathrm{sp}^{2}: \mathrm{sp}^{3}$ ratio to a certain degree, all interactions above $2.25 \AA$ are still disregarded. This can have a serious impact on the local first and second neighbor environments. The tight binding structure optimizations, on the other hand, modify the surface regions of the cut out samples to impose the periodicity needed for the DFT calculations. The ensuing atomic rearrangement is unphysical, strictly speaking, as it is completely disconnected from the track formation procedure. An alternative would be a full MD simulation on these samples including the respective heat and quench cycles. Timescales and temperatures could be extracted from the empirical potential MD simulations of the track formation and a-C synthesis runs. Ideally these simulations should be performed as ab initio calculations. To reproduce the surface sensitivity of STS new DFT calculations on thin surface samples could be performed. A closer agreement between such simulations and the STS measurements is expected.

Current-voltage spectroscopy experiments proved to be problematic. The presented temperature-dependent measurements constitute an averaging over an ensemble of many tracks. Due to embedded nature of the tracks four-point measurements cannot easily be performed. Therefore, it is difficult to separate interface effects at both the metalized top contact and the silicon or metalized substrate. Smoothly transient conduction regimes were identified, but, as can be expected from the approximative nature of the known conduction models, no single model that fits the entire range of temperatures and electrical fields could be determined. The conduction properties of an average track do not necessarily equal the averaged conduction properties of many tracks. This is in fact only the case if the physical properties contribute 


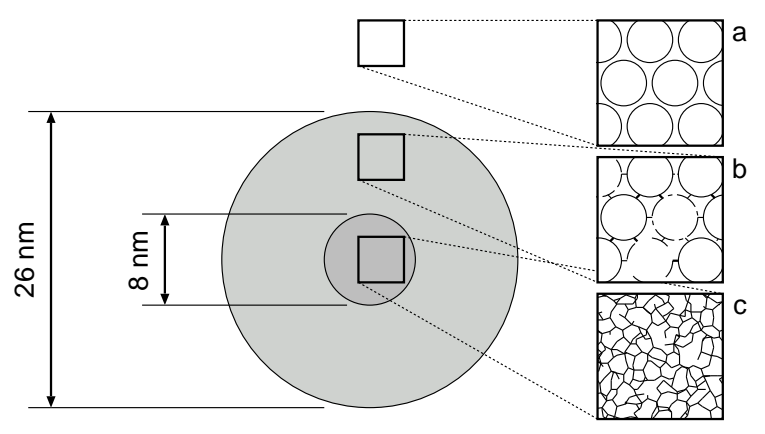

Figure 4.2: $\quad$ Raman scattering and AFM measurements suggest a two zone track model for fullerene films. Embedded in a matrix composed of pristine $\mathrm{C}_{60}$ molecules (a) an outer shell of defective fullerene molecules (b), polymerized to a varying degree, with fully amorphized material in the core (c) make up each ion track. Neither polymerized shell, nor amorphized core exhibit a fullerene $A_{g}(2)$ Raman signal, but only the fully amorphized core shows up on the C-AFM measurements.

linearly to the conduction mechanism. Thus, a single track contact is desirable. A technique to create single ion track contacts has been proposed and implemented to a certain degree. A polycarbonate masking process was developed and the ensuing steps of track etching and filling were successfully demonstrated.

The seemingly contradictory values for track diameters extracted from Raman scattering and AFM measurements can be explained by a simple umbra and penumbra model (fig. 4.2). It describes the ion track as a two zone coaxial system. An umbra in the core region with fully amorphized material and no fullerene structure remaining, enclosed by a penumbra region containing defective $\mathrm{C}_{60}$ molecules polymerized to a varying degree. The lateral force and C-AFM signals are sensitive to the completely amorphized track center region. The material must be fully amorphized to create a current path, and modify the surface roughness. The peripheral region is likely to be formed by delta electrons which carry energy away from the track core. Fullerene films are sensitive to electron irradiation, which causes polymerization by linking neighboring $\mathrm{C}_{60}$ molecules. The Raman signal is very sensitive to this morphological modification and hence yields a larger track radius.

The abundance of stellar hydrogen and its interaction with inter-planetary dust grains and the observed ion track formation in plasma deposited hydrogen rich carbon samples call for simulations of hydrogenated amorphous carbon (a-C:H) 
and tetrahedrally bound amorphous carbon (ta-C:H) samples. The low mass of the hydrogen atoms causes an excitation to high velocities and a possible removal from the track core zone. However, the high hydrogen atom velocities demand small simulation timesteps and increase the computational cost per simulated time-span. Simulations of fullerene films with respect to formation of surface features could help explain the friction increase and shed light on the structure of the amorphous carbon material in the track cores. However, the inter molecular binding in those films is mediated by van der Waals dispersion forces. Their long range makes such computer simulations computationally intensive.

Lastly the study of ion irradiation effects on small particles is a promising task. Due to their confined geometry very little heat dissipation can occur, potentially amplifying the sensitivity to morphological changes. Preliminary simulation runs show great promise, displaying sputtering effects which could lead to possible formation of complex carbon molecules.

The viability of a thermal spike treatment of ion tracks in amorphous carbon molecular dynamics simulations shown in this work provides a powerful tool to approach these interesting tasks. 


\section{Appendix A}

\section{Current Distribution}

To facilitate the computation of the current distribution in a given sample it can be approximated by a three-dimensional resistor network, discretizing the conduction properties of the continuum with a finite number $n$ of resistors. Conductances between two nodes $i$ and $j$ in the network are stored in a $n \times n$ matrix $\hat{\sigma}$, the connectivity-matrix of the network.

(A.1).

$\hat{C}=\hat{\sigma}-\hat{\varphi}=\left(\begin{array}{cccccc}-\varphi_{1} & \sigma_{1,2} & \cdots & \sigma_{1,1+n x} & \\ \sigma_{2,1} & -\varphi_{2} & \sigma_{2,3} & & \sigma_{1,1+n x} \\ \vdots & \sigma_{3,2} & -\varphi_{3} & \ddots & & \ddots \\ \sigma_{1+n y, 1} & & \ddots & \ddots & \\ & \sigma_{2+n y, 2} & & & \\ & & \ddots & & \\ & & \hat{B} & & \end{array}\right) \quad, \quad \vec{V}=\left(\begin{array}{c}V_{1} \\ \vdots \\ V_{n}\end{array}\right)$

The $n \times n$ diagonal matrix $\hat{\varphi}$ with elements $\varphi_{i}$ consisting of the sums of all conductances leading to the node $i$ is introduced to convert potentials to potential 
differences. The vector $\vec{V}$ denotes the potentials $V_{i}$ at node $i$. With $\hat{B}$ being empty $\hat{C} \cdot \vec{V}$ yields a vector containing $n$ terms of the form

$$
-\underbrace{\sum_{j \in N_{i}} C_{i, j}}_{\varphi_{i}} V_{i}+\sum_{j \in N_{i}}\left(C_{i, j} V_{j}\right)
$$

$N_{i}$ being the set of nodes adjacent to $i$. With $V_{i}-V_{j}=U_{i, j}$ this can be transformed to the sum of currents entering node $i$

$$
\sum_{j \in N_{i}}\left(\sigma_{i, j} U_{i, j}\right)=\sum_{j \in N_{i}} I_{i, j}
$$

To comply with Kirchhoff's second law this sum has to be zero. In the vector notation the following equation has to be solved.

$$
\hat{C} \cdot \vec{V}=\overrightarrow{0}_{n}
$$

Without further boundary conditions this constitutes an $n$-dimensional homogeneous system of linear equations, with a kernel of potential vectors $\vec{V}$ assigning each node the same potential. A number $b$ of boundary conditions can be introduced using the $b \times n$ block matrix $\hat{B}$. Setting elements $B_{i, m(i)}$ to 1 , with the injective function $m$ that maps the boundary condition index $i$ to the node number $m(i)$

With the definition of a new vector

$$
\vec{Y}=\left(\begin{array}{c}
\overrightarrow{0}_{n} \\
\vec{Z}
\end{array}\right)
$$

composed of $n$ zero elements and a vector $\vec{Z}$ with $b$ elements $Z_{i}$ containing the potential for node $j(i)$, equation A.3 can be rewritten as

$$
\hat{C} \cdot \vec{V}=\vec{Y}
$$

This yields $b$ additional terms to eq. A.3 of the form

$$
V_{i}=Z_{i}
$$

containing boundary conditions on the potential at certain nodes. 


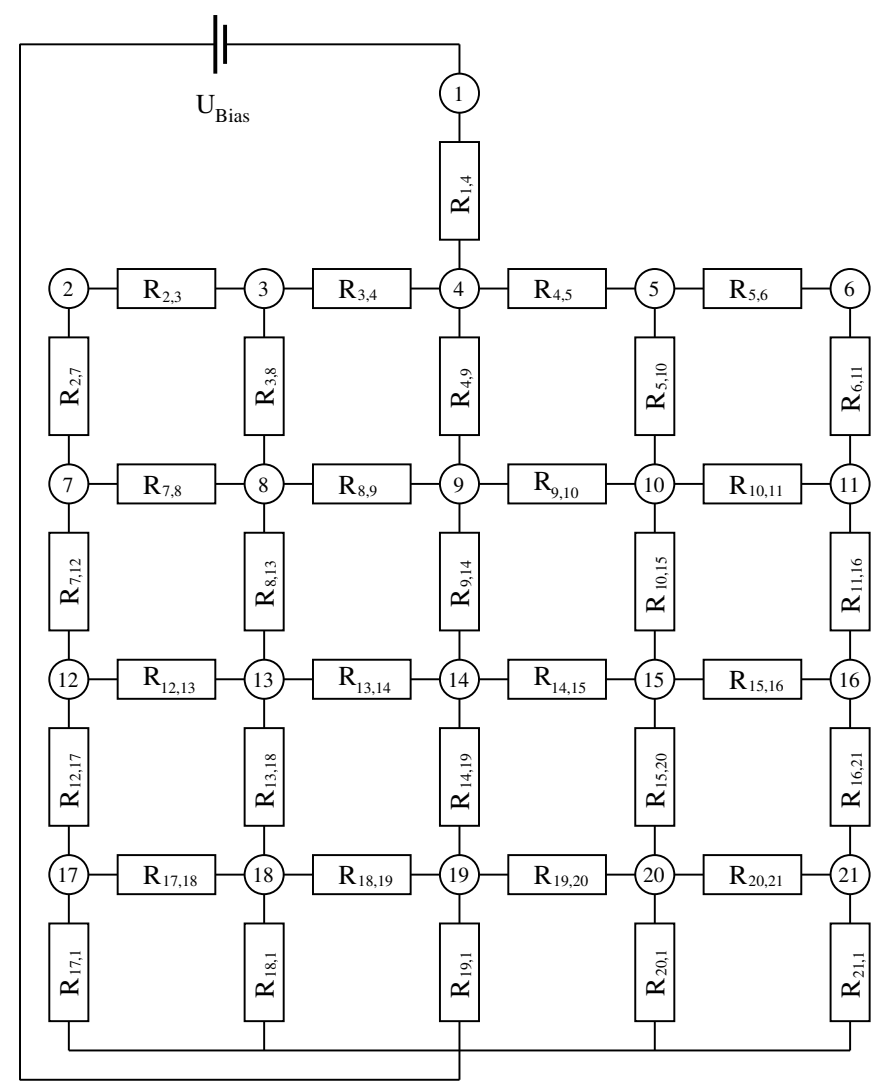

Figure A.1: Connection matrix ansatz used to solve for the current distribution in the unirradiated and irradiated samples. The track is modeled by increasing the conductivity of all vertical connections in the center column.

Equation A.5 can be solved for $\vec{V}$ using a computer algebra system, yielding a potential map of the system. The current between node $i$ and node $j$ are simply given by $\sigma_{i, j} \cdot\left(V_{i}-V_{j}\right)$. The current flow at each node was computed by summing the incoming and outgoing currents divided by two. 


\section{Appendix B}

\section{Publications}

\section{B.1 List of publications}

1. Lattice Location Studies of Indium in $\mathrm{Cr}_{2} \mathrm{O}_{3}$, U. Vetter, M. Uhrmacher, D. Schwen, A. Lohstroh, H. Hofsäss, and K.-P. Lieb, Hyperfine Interactions, 136-137 3-8 (2001) 607-612

2. Ion beam synthesis of boron carbide thin films, C. Ronning, D. Schwen, S. Eyhusen, U. Vetter, and H. Hofsäss, Surface and Coatings Technology 158 (2002) 382

3. Field emission enhancement by graphitic nano-scale channels through ta-C layers, N. Koenigsfeld, H. Hofsäss, D. Schwen, A. Weidinger, C. Trautmann, and R. Kalish, Diamond and Related Materials 12 (2003) 469

4. Conductivity of Ion tracks in diamond-like carbon films, J.-H. Zollondz, J. Krauser, A. Weidinger, C. Trautmann, D. Schwen, C. Ronning, H. Hofsäss, and B. Schultrich, Diamond and Related Materials 12 (2003) 938

5. Fieldemission studies on swift heavvy ion irradiated amorphous carbon, D. Schwen, C. Ronning, and H. Hofsäss, Diamond and Related Materials 13 (2004) 1032

6. Manganese doped ZnO nanobelts for spintronics, C. Ronning, P.X. Gao, Y. Ding, Z.L. Wang, and D. Schwen, Applied Physics Letters 84 (2004) 783 
7. Quasi One-Dimensional Electrical Conductors Created by Swift Heavy Ion Tracks in Tetrahedral Amorphous Carbon Films, D. Schwen, H. Hofsäss, A.K. Nix, H. Krauser, C. Trautmann, GSI Scientific Report 2005, GSI Report 2006-1

8. Catalyst-Nanostructure Interaction in the Growth of 1-D ZnO Nanostructures, C. Borchers, S. Müller, D. Stichtenoth, D. Schwen, and C. Ronning, J.Phys.Chem.B 110 (2006) 1656

9. Catalyst nanostructure interaction and growth of $Z n S$ nanobelts, C. Borchers, D. Stichtenoth, S. Müller, D. Schwen, and C. Ronning, Nanotechnology 17 (2006) 1067-1071

10. Conductive nanoscopic ion-tracks in diamond-like-carbon, J.-H. Zollondz, D. Schwen, A.-K. Nix, et al., Mater. Sci. Eng. C 26 (2006) 1171-1174

11. Optical activation of implanted impurities in $Z n S$ nanowires, D. Stichtenoth, D. Schwen, S. Müller et al., J. Vac. Sci. Tech. A 24 (2006) 1356-1359

12. Electronic properties of graphite-like ion tracks in insulating tetrahedral amorphous carbon, A.-K. Nix, D. Schwen, C. Ronning, J. Krauser, C. Trautmann, and H. Hofsäss, Physica Status Solidi (B), to be published

13. Morphological change of carbon surfaces by sputter erosion, K. Takahiro1, K. Zhang, F. Rotter, D. Schwen, C. Ronning, H. Hofsss, J. Krauser, Nucl. Instr. and Meth B (2007), in press

14. Atomistic simulations of swift ion tracks in diamond and graphite, D. Schwen and E. Bringa, Nucl. Instr. and Meth B (2007), in press

15. Ultrafast carrier dynamics in tetrahedral amorphous carbon film: carrier trapping vs. electron-hole combination, E. Carpene, E. Mancini, C. Dallera, S. De Silvestri, D. Schwen, C. Ronning, Phys. Rev. Lett, submitted 


\section{B.2 Conference contributions}

1. DPG 2002, Regensburg, Talk: Untersuchungen zur Feldemission an Ionenspuren in Kohlenstoffschichten, D. Schwen, C. Ronning, H. Hofsäss, J. Krauser, J.-H. Zollondz, A. Weidinger, and C. Trautmann.

2. KFSI Bonn 2002, Talk: Untersuchungen zur Feldemission von Ionenspuren in diamantähnlichem Kohlenstoff, D. Schwen, C. Ronning, H. Hofsäss, J.-H. Zollondz, J. Krauser, A. Weidinger, and C. Trautmann.

3. DPG 2003, Dresden, Poster: Nanostructured diamond-like carbon for field emission applications, D. Schwen, C. Ronning, H. Hofsäss, J.-H. Zollondz, A. Weidinger, and C. Trautmann.

4. Diamond 2003, Salzburg, Poster: Conducting ion tracks in diamond like carbon, D. Schwen, C. Ronning, H. Hofsäss, J.-H. Zollondz, A. Weidinger, and C. Trautmann.

5. EUNITT 2004, Uppsala, Talk: Field emission studies on swift heavy ion irradiated tetrahedral amorphous carbon (ta-C), D. Schwen, C. Ronning, H. Hofsäss, J.-H. Zollondz, and A. Weidinger.

6. NANO-8 2004, Venedig, Poster: Synthesis and Properties of ZnS Nanobelts, D. Schwen, D. Stichtenoth, T. Hernan, S. Müller, C. Borchers, M. Seibt, H. Hofsäss, and C. Ronning.

7. IBMM 2004, Monterey, Poster: Electrical properties of tracks in swift heavy ion irradiated tetrahedral amorphous carbon, D. Schwen, A.-K. Nix, H. Hofsäss, H. Zollondz, A. Weidinger, C. Trautmann, and J. Krauser.

8. SHIM 2005, Aschaffenburg, Poster: Electrical properties of tracks in swift heavy ion irradiated tetrahedral amorphous carbon, D. Schwen, A.-K. Nix, C. Ronning, H. Hofsäss, J.-H. Zollondz, C. Trautmann, and J. Krauser.

9. SPP Meeting 2005, Fulda, Poster: Ion Implantation Doping of Nanowires, D. Schwen, D. Stichtenoth, S. Müller, C. Borchers, and C. Ronning. 
10. Nanomat 2005, Karlsruhe, Poster: Semiconductor Nanowires, D. Schwen, D. Stichtenoth, S. Müller, C. Borchers, and C. Ronning. (poster award)

11. FAHL Academy 2005, Wörlitz, Poster: Ion beam doping of semiconductor nanowires, D. Stichtenoth, S. Müller, D. Schwen, C. Borchers, and C. Ronning.

12. KFSI Workshop 2005, Munich, Talk : Electronic Properties of Conducting Ion Tracks in ta-C, D. Schwen, A.-K. Nix, J. Krauser, C. Trautmann, and H. Hofsäss.

13. MRS 2005, Boston, Talk: Electronic Properties of Conducting Ion Tracks in ta-C, D. Schwen, A.-K. Nix, J. Krauser, C. Trautmann, and H. Hofsäss.

14. SNI 2006, Hamburg, Talk: Quasi One-Dimensional Electrical Conductors Created by Swift Heavy Ion Tracks in Tetrahedral Amorphous Carbon Films, D. Schwen, A.-K. Nix, C. Ronning, J. Krauser, C. Trautmann, and E. Bringa. 


\section{Appendix C}

\section{List of Abbreviations}

a-C amorphous carbon

a-C:H hydrogenated amorphous carbon

AFM atomic force microscopy

AIREBO adaptive intermolecular reactive empirical bond order (potential)

C-AFM conducting atomic force microscopy

CDF conical dark field

CTF contrast transfer function

DFT density functional theory

DLC diamond-like carbon

DOS density of states

EDIP environment-dependent interatomic potential

EELS electron energy loss spectroscopy

FFT fast fourier transformation

FWHM full width at half maximum

HOPG highly oriented pyrolytic graphite

LDOS local density of states

MSIBD mass selected ion-beam deposition

REBO reactive empirical bond order (potential)

RMS root mean square

SEM scanning/secondary electron microscopy

STM scanning tunneling microscopy

STS scanning tunneling spectroscopy

ta-C tetrahedrally bound amorphous carbon

ta-C:H hydrogenated tetrahedrally bound amorphous carbon

TEM transmission electron microscopy

REBO reactive bond order

UHV ultra high vacuum

ZBL Ziegler-Biersack-Littmark 


\section{List of Figures}

1.1 Stopping powers in ta-C . . . . . . . . . . . . 8

1.2 Ta-C film with a density of about $\approx 3 \mathrm{~g} / \mathrm{cm}^{3}$, irradiation perpendicular to film surface. . . . . . . . . . . . . . . . . . . . 99 9

1.3 Swift heavy ion passes within $\approx 1 / 100 \mathrm{fs}$, leaving a trail of high energetic electronic excitations. . . . . . . . . . . . . . 9 9

1.4 Electronic excitations couple to the lattice, heating it up, energy is available for atomic rearrangement. . . . . . . . . . . . 9

1.5 Density is reduced, $\mathrm{sp}^{2}$-fraction increased, heat dissipates within ps. $\quad 9$

1.6 Differential reflectivity and free carrier dynamics in ta-C . . . . . 11

1.7 Differential reflectivity at early times . . . . . . . . . . . . . . 12

1.8 Energyband schematics . . . . . . . . . . . . . . . . . . . . 14

1.9 Frenkel-Poole energy barrier schematics . . . . . . . . . . . . . . 15

1.10 Simulated current distributions . . . . . . . . . . . . . . . 19

1.11 Measured conductivity contrast . . . . . . . . . . . . . . 20

2.13 D rendering of an amorphous carbon network . . . . . . . . 25

2.2 Optimization schemes . . . . . . . . . . . . . . 26

2.3 Bulkmoduli of ta-C and diamond . . . . . . . . . . . . . . . 29

2.4 Thermal spike approximation of an ion track . . . . . . . . . . . . . 32

2.5 Diamond bond plot . . . . . . . . . . . . . . . . . . 33

2.6 Graphite bond plot . . . . . . . . . . . . . . . 34

2.7 Neighbor distribution functions . . . . . . . . . . . . . . 36

2.8 Radialy averaged hybridization distributions . . . . . . . . . . . 38

2.9 Radial temperature distribution . . . . . . . . . . . . . . . . . . 39

2.10 Free surface simulation . . . . . . . . . . . . . . . . . 41 
2.11 Hillock heights and radii . . . . . . . . . . . . . . . . . . 42

2.12 Calculated DOS for diamond and graphite . . . . . . . . . . . . . 45

2.13 Calculated averaged DOS for irradiated amorphous carbon . . . . . 47

2.14 Image simulation schematic . . . . . . . . . . . . . . . . . . . . 49

2.15 Simulated TEM images for a modeled track density distribution . . 51

2.16 Simulated TEM images of a-C MD data sets . . . . . . . . . . . . 53

2.17 Simulated TEM images of diamond MD datasets . . . . . . . . . 54

3.1 AFM topography . . . . . . . . . . . . . . . 56

3.2 AFM current measurements . . . . . . . . . . . . . . 58

3.4 Hillocks on Xe irradiated ta-C . . . . . . . . . . . . . . . 58

3.3 Electronic stopping power curves for selected ion species . . . . . . 59

3.5 Hillock height histogram . . . . . . . . . . . . . . . . . . . . 60

3.6 Measuring DOS with the STM . . . . . . . . . . . . . 61

3.7 STM topography measurement . . . . . . . . . . . . 63

3.8 Crosssectional TEM image, ta-C on $\mathrm{Fe}$. . . . . . . . . . . . . 64

3.9 STM current maps at $0.8 \mathrm{~V}$ and $1.0 \mathrm{~V}$ bias voltage . . . . . . . . 65

3.10 STM integral current map . . . . . . . . . . . . . . . . . . . 66

3.11 LDOS sampling locations . . . . . . . . . . . . . . . 67

3.12 LDOS curves on- and off-track . . . . . . . . . . . . . . . . . 68

3.13 IV sample setup . . . . . . . . . . . . . . . . . . . . . . 69

3.14 Conduction regimes . . . . . . . . . . . . . . . . . 70

3.15 Comparison of variable range hopping and Frenkel-Poole conduction 71

3.16 Polycarbonate . . . . . . . . . . . . . . . . 72

3.17 Polycarbonate . . . . . . . . . . . . . . . . 73

3.19 Etched $\mathrm{PC}$ mask . . . . . . . . . . . . . . . . . . . . . . 74

3.18 Track etching . . . . . . . . . . . . . . . . . . . . . 74

3.20 Single contact process _. . . . . . . . . . . . . . . 76

3.21 Wet chemical substrate removal . . . . . . . . . . . . . . 77

3.22 Carbon foil thickness . . . . . . . . . . . . . . . . . . . 77

3.23 Plane view TEM parallel . . . . . . . . . . . . . . . . . . 78

3.24 Plane view TEM tilted . . . . . . . . . . . . . . . . . . . . . . . 79

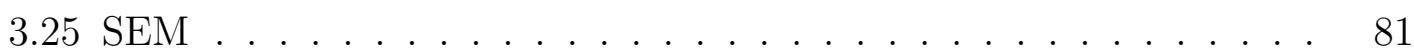


3.26 Raman signal of irradiated $\mathrm{C}_{60}$ films $\ldots \ldots$. . . . . . . . 83

3.27 Comparison of lateral force and C-AFM images on $\mathrm{C}_{60} \ldots \ldots 4$

4.1 Comparison of simulated and experimental hillock heights _ . . . 89

4.2 Proposed $\mathrm{C}_{60}$ track model . . . . . . . . . . . . . . . 92

A.1 Connection matrix f . . . . . . . . . . . . . . . . . . . 97 


\section{Bibliography}

[Ale97] H. Alexander, Physikalische Grundlagen der Elektronenmikroskopie, B. G. Teubner, 1 edn. (1997).

[Ama95] G. A. J. Amaratunga, J. Robertson, V. S. Veerasamy, W. I. Milne, and D. R. McKenzie, Gap states, doping and bonding in tetrahedral amorphous carbon, Diamond Relat. Mater. 4(5-6), 637 (1995).

[And58] P. W. Anderson, Absence of Diffusion in Certain Random Lattices, Phys. Rev. 109(5), 1505 (1958).

[Ape03] P. Apel, Swift ion effects in polymers: industrial applications, Nucl. Instrum. Methods Phys. Res., Sect. B 208, 11 (2003).

[Ban89] J. Banhart, P. Weinberger, and J. Voitländer, Short-range-order effects in $C u_{x} P t_{1-x}$, Phys. Rev. B 40(18), 12079 (1989).

[Bet28] H. A. Bethe, Theorie der Beugung von Elektronen an Kristallen, Ann. d. Physik 87, 55 (1928).

[Beu03] M. Beuve, N. Stolterfoht, M. Toulemonde, C. Trautmann, and H. M. Urbassek, Influence of the spatial and temporal structure of the depositedenergy distribution in swift-ion-induced sputtering, Phys. Rev. B 68, 125423 (2003).

[Bin82] G. Binnig, H. Rohrer, Ch. Gerber, and E. Weibel, Tunneling through a controllable vacuum gap, Appl. Phys. Lett. 40(2), 178 (1982).

[Bin86] G. Binnig, C. F. Quate, and Ch. Gerber, Atomic Force Microscope, Phys. Rev. Lett. 56, 930 (1986).

[Bol06] T. Bolse, A. Elsanousi, H. Paulus, and W. Bolse, Dewetting of nickel oxidefilms on silicon under swift heavy ion irradiation, Nucl. Instrum. Methods Phys. Res., Sect. B 244(1), 115 (2006). 
[Bra84] J. P. Bradley, D. E. Brownlee, and P. Fraundorf, Discovery of Nucler Tracks in Interplanetary Dust, Science 226(4681), 1432 (1984).

[Bre90] D. W. Brenner, Empirical potential for hydrocarbons for use in simulating the chemical vapor deposition of diamond films, Phys. Rev. B 42, 9458 (1990), corrigendum Phys.Rev. B 46, 1948 (1992).

[Bri99] E. M. Bringa, R. E. Johnson, and M. Jakas, Molecular-dynamics simulations of electronic sputtering, Phys. Rev. B 60, 15107 (1999).

[Bri02a] E. M. Bringa and R. E. Johnson, Coulomb Explosion and Thermal Spikes, Phys. Rev. Lett. 88, 165501 (2002).

[Bri02b] E. M. Bringa, R. Papaleo, and R. E. Johnson, Crater formation by single ions in the electronic stopping regime: Comparison of molecular dynamics simulations with experiments on organic films, Phys. Rev. B 65, 094113 (2002).

[Bus93] R. H. Buss, A. G. G. M. Tielens, M. Cohen, M. W. Werner, J. D. Bregman, and F. C. Witteborn, Infrared spectra of transition objects and the composition and evolution of carbon dust, Astrophys J. 415, 250 (1993).

[Car81] M. J. Cardillo, Nature of the Si(111) $7 \times 7$ reconstruction, Phys. Rev. $B$ 23(8), 4279 (1981).

[Car85] R. Car and M. Parrinello, Unified Approach for Molecular Dynamics and Density-Functional Theory, Phys. Rev. Lett. 55(22), 2471 (1985).

[Car07] E. Carpene, E. Mancini, C. Dallera, S. De Silvestri, D. Schwen, and C. Ronning, Ultrafast carrier dynamics in tetrahedral amorphous carbon film: carrier trapping vs. electron-hole combination, Phys. Rev. Lett. (2007), submitted.

[Coh69] M. H. Cohen, H. Fritzsche, and S. R. Ovshinsky, Simple Band Model for Amorphous Semiconducting Alloys, Phys. Rev. Lett. 22(20), 1065 (1969).

[Cow57] J. M. Cowley and A. F. Moodie, The Scattering of Electrons by Atoms and Crystals. I. A New Theoretical Approach, Acta Cryst. 10, 609 (1957).

[Cro70] G. B. Crooks and M. E. Rudd, Experimental Evidence for the Mechanism of Charge Transfer into Continuum States, Phys. Rev. Lett. 25(23), 1599 (1970). 
[Cro74] G. Crozaz, R. Drozd, C. Hohenberg, C. Morgan, C. Ralston, R. Walker, and D. Yuhas, Lunar surface dynamics - Some general conclusions and new results from Apollo 16 and 17, in Lunar Science Conference, 5th, Houston, Tex., p. 2475, Pergamon Press (1974).

[Dob01] D. Dobrev, J. Vetter, N. Angert, and R. Neumann, Growth of iron single crystals in the etched ion tracks of polymer foils, Appl. Phys. A 72(6), 729 (2001).

[Doy67] P. A. Doyle and P. S. Turner, Relativistic Hartree-Fock X-ray and Electron Scattering Factors, Acta Cryst. A24, 390 (1967).

[Dre96] M. S. Dresselhaus, G. Dresselhaus, and P. C. Eklund, Raman Scattering in Fullerenes, J. Raman Spec. 27(3-4), 351 (1996).

[Duc91] S. J. Duclos, R. C. Haddon, S. H. Glarum, A. F. Hebard, and K. B. Lyons, The influence of oxygen on the Raman spectrum of $C_{60}$ films, Solid State Commun. 80(7), 451 (1991).

[Enc03] I. Enculescu, Z. Siwy, D. Dobrev, C. Trautmann, M. E. Toimil Molares, R. Neumann, K. Hjort, L. Westerberg, and R. Spohr, Copper nanowires electrodeposited in etched single-ion track templates, Appl. Phys. A 77(6), 751 (2003).

[Eyn92] G. v. Eynatten, K. Nothelfer, and K. Dransfeld, Light induced chemical reactions on interfaces between metallic iron and polymers, Hyperfine Interact. 69(1-4), 759 (1992).

[Fal93] P. J. Fallon, V. S. Veerasamy, C. A. Davis, J. Robertson, G. A. J. Amaratunga, W. I. Milne, and J. Koskinen, Properties of filtered-ion-beamdeposited diamondlike carbon as a function of ion energy, Phys. Rev. B 48(7) (1993).

[Fer93] E. Ferain and R. Legras, Heavy ion tracks in polycarbonate. Comparison with a heavy ion irradiated model compound (diphenyl carbonate), Nucl. Instrum. Methods Phys. Res., Sect. B 82(4), 539 (1993).

[Fle64a] R. L. Fleischer and P. B. Price, Techniques for geological dating of minerals by chemical etching of fission fragment tracks, Geochim. Cosmochim. Acta 28(10), 1705 (1964).

[Fle64b] R. L. Fleischer, P. B. Price, R. M. Walker, and E. L. Hubbard, Track Registration in Various Solid-State Nuclear Track Detectors, Phys. Rev. 133, A1443 (1964). 
[Fle67] R. L. Fleischer, P. B. Price, and R. M. Walker, Criterion for Registration in Dielectric Track Detectors, Phys. Rev. 156(2), 353 (1967).

[Fle75] R. L. Fleischer, P. B Price, and R. M. Walker, Nuclear Tracks in Solids, Univ. California Press, Berkeley (1975).

[Fre38] J. Frenkel, On Pre-Breakdown Phenomena in Insulators and Electronic Semi-Conductors, Phys. Rev. 54(80), 647 (1938).

[Glo99] J. N. Glosli and F. H. Ree, The melting line of diamond determined via atomistic computer simulations, J. Appl. Phys. 110, 441 (1999).

[Gon02] X. Gonze, J.-M. Beuken, R. Caracas, F. Detraux, M. Fuchs, G.-M. Rignanese, L. Sindic, M. Verstraete, G. Zerah, F. Jollet, M. Torrent, A. Roy, M. Mikami, Ph. Ghosez, J.-Y. Raty, and D.C. Allan, First-principles computation of material properties: the ABINIT software project, Comput. Mater. Sci. 25, 478 (2002).

[Ham86] R. J. Hamers, R. M. Tromp, and J. E. Demuth, Surface Electronic Structure of Si (111)-(7 × 7) Resolved in Real Space, Phys. Rev. Lett. 56(18), 1972 (1986).

[Han06] M. J. Han, T. Ozaki, and J. Yu, $O(N) L D A+U$ electronic structure calculation method based on the nonorthogonal pseudoatomic orbital basis, Phys. Rev. B 73, 045110 (2006).

[Her98] O. Herre, W. Wesch, E. Wendler, P. I. Gaiduk, F. F. Komarov, S. Klaumünzer, and P. Meier, Formation of discontinuous tracks in singlecrystalline InP by 250-MeV Xe-ion irradiation, Phys. Rev. B 58(8), 4832 (1998).

[HN05] S.R. Hashemi-Nezhad, The geometry of etched heavy ion tracks in phlogopite mica; a clear dependence on energy deposited, Nucl. Instrum. Methods Phys. Res., Sect. B 234(4), 533 (2005).

[Hof93] H. Hofsäss, H. Binder, T. Klumpp, and E. Recknagel, Doping and growth of diamond-like carbon films by ion beam deposition, Diamond Relat. Mater. 3(1-2), 137 (1993).

[Hof98] H. Hofsäss, H. Feldermann, R. Merk, M. Sebastian, and C. Ronning, Cylindrical spike model for the formation of diamondlike thin films by ion deposition, Appl. Phys. A 66, 153 (1998). 
[Hoh64] P. Hohenberg and W. Kohn, Inhomogeneous Electron Gas, Phys. Rev. 136(3B), B864 (1964).

[Hur91] A. J. Hurford, Uplift and cooling pathways derived from fission track analysis and mica dating: a review, Int. J. Earth Sci. 80(2), 349 (1991).

[IO00] V. I. Ivanov-Omskii, A. B. Lodygin, and S. G. Yastrebov, The Scanning Tunneling Microscopy and Scanning Tunneling Spectroscopy of Amorphous Carbon, Semiconductors 34(12), 1355 (2000).

[Jäg00] H. U. Jäger and K. Albe, Molecular-dynamics simulations of steady-state growth of ion-deposited tetrahedral amorphous carbon films, J. Appl. Phys. 88, 1129 (2000).

[Jak02] M. Jakas, E. M. Bringa, and R. E. Johnson, Fluid dynamics calculation of sputtering from a cylindrical thermal spike, Phys. Rev. B 65, 165425 (2002).

[Jen00] J. Jensen and P. Sigmund, Electronic stopping of swift partially stripped molecules and clusters, Phys. Rev. A 61(3), 032903 (2000).

[Jes01] H. O. Jeschke, M. E. Garcia, and K. H. Bennemann, Theory for the Ultrafast Ablation of Graphite Films, Phys. Rev. Lett. 87, 015003 (2001).

[Joh80] E. Johnson and R. Evatt, Thermal Spikes and Sputtering Yields, Radiat. Eff. 52, 187 (1980).

[Joh93a] M. B. Johnson, O. Albrektsen, R. M. Feenstra, and H. W. M. Salemink, Direct imaging of dopants in GaAs with cross-sectional scanning tunneling microscopy, Appl. Phys. Lett. 63(21), 2923 (1993).

[Joh93b] R. E. Johnson and J. Schou, Sputtering of Organic Insulators, Mat. Fys. Medd. K. Dan. Vidensk. Selsk. 43, 403 (1993).

[Kat69] R. Katz and E. J. Kobetich, Particle Tracks in Emulsion, Phys. Rev. 186, 344 (1969).

[Ker99] M. Kerford and R. P. Webb, Molecular Dynamics simulation of the desorption of molecules by energetic fullerene impacts on graphite and diamond surfaces, Nucl. Instrum. Methods Phys. Res., Sect. B 153, 270 (1999).

[Koh65] W. Kohn and L. J. Sham, Self-Consistent Equations Including Exchange and Correlation Effects, Phys. Rev. 140(4A), A1133 (1965). 
[Kol95] A. A. Kolomenskii, M. Szabadi, and P. Hess, Laser diagnostics of $C_{60}$ and $C_{70}$ films by broadband surface acoustic wave spectroscopy, Appl. Surf. Sci. 86(1-4), 591 (1995).

[Krä90] W. Krätschmer, L. D. Lamb, F. Fostiropoulos, and D. R. Huffman, Solid $C_{60}$ : a new form of carbon, Nature 347, 354 (1990).

[Kra93] B. Kramer and A. MacKinnon, Localization: theory and experiment, Rep. Prog. Phys. 56, 1469 (1993).

[Kra01] J. Krauser, V. Hoffmann, W. Harneit, M. Waiblinger, N. Stolterfoht, A. Weidinger, C. Trautmann, H. Hofsäss, C. Ronning, B. Schultrich, and H. Sturm, Graphitic nanowires embedded in diamond-like carbon films, AIP Conf. Proc. 591, 507 (2001).

[Kra03] J. Krauser, J.-H. Zollondz, A. Weidinger, and C. Trautmann, Conductivity of nanometer-sized ion tracks in diamond-like carbon ?lms, J. Appl. Phys. 94(3), 1959 (2003).

[Kro85] H. W. Kroto, J. R. Heath, S. C. O'Brien, R. F. Curl, and R. E. Smalley, $C_{60}$ : Buckminsterfullerene, Nature 318, 162 (1985).

[Kub83] O. Kubaschewski and C. B. Alcock, Metallurgical Thermochemistry, Pergamon Press, Oxford, 5 edn. (1983).

[Lan86] N. D. Lang, Spectroscopy of single atoms in the scanning tunneling microscope, Phys. Rev. B 34(8), 5947 (1986).

[Lan00] D. Landau and K. Binder, A guide to Monte Carlo Simulations in Statistical Physics, Cambridge University Press, Cambridge (2000).

[Lan05] M. Lang, U. A. Glasmacher, R. Neumann, D. Schardt, C. Trautmann, and G. A. Wagner, Energy loss of 50-GeV uranium ions in natural diamond, Appl. Phys. A (80), 681 (2005).

[Liu01] J. Liu, R. Neumann, C. Trautmann, and C. Müller, Tracks of swift heavy ions in graphite studied by scanning tunneling microscopy, Phys. Rev. B (64), 184115 (2001).

[Lon99] M. Lonfat, B. Marsen, and K. Sattler, The energy gap of carbon clusters studied by scanning tunneling spectroscopy, Chem. Phys. Lett. 313, 539 (1999). 
[Mal88] T. Malis, S. C. Cheng, and R. F. Egerton, EELS Log-Ratio Technique for Specimen-Thickness Measurement in the TEM, J. El. Micr. Tech. 8, 193 (1988).

[Man01] C. Manfredotti, E. Vittone, A. Lo Giudice, C. Paolini, F. Fizzotti, G. Dinca, V. Ralchenko, and S. V. Nistor, Ionoluminescence in CVD diamond and in cubic boron nitride, Diamond Relat. Mater. 10(3-7), 568 (2001).

[Mar90] N. Maron, Optical properties of fine amorphous carbon grains in the infrared region, Astrophys Space Science 172, 21 (1990).

[Mar97] N. A. Marks, Evidence for subpicosecond thermal spikes in the formation of tetrahedral amorphous carbon, Phys. Rev. B 56, 2441 (1997).

[Mar02] N. A. Marks, N. C. Cooper, D. R. McKenzie, D. G. McCulloch, P. Bath, and S. P. Russo, Comparison of density-functional, tight-binding, and empirical methods for the simulation of amorphous carbon, Phys. Rev. B 65, 075411 (2002).

[Mar04] A. Mara, Z. Siwy, C. Trautmann, J. Wan, and F. Kamme, An asymmetric polymer nanopore for single molecule detection, Nano Lett. 4(3), 497 (2004).

[Mar05] R. M. Martin, Electronic Structure, Basic Theory and Practical Methods, Cambridge University Press, 1 edn. (2005).

[Mar06] J. Marian, L. A. Zepeda-Ruiz, G. H. Gilmer, E. M. Bringa, and T. Rognlien, Simulations of carbon sputtering in amorphous hydrogenated samples, Physica Scripta T124, 65 (2006).

[Mat93] M. Matus and H. Kuzmany, Raman Spectra of Single-Crystal C60, Appl. Phys. A 56(3), 241 (1993).

[McK91] D. R. McKenzie, D. Muller, and B. A. Pailthorpe, Compressive-StressInduced Formation of Thin-Film Tetrahedral Amorphous Carbon, Phys. Rev. Lett. 67(6), 773 (1991).

[McS57] H. J. McSkimin and W. L. Bond, Elastic Moduli of Diamond, Phys. Rev. 105(1), 116 (1957). 
[Mef94] A. Meftah, F. Brisard, J. M. Costantini, E. Dooryhee, M. Hage-Ali, M. Hervieu, J. P. Stoquert, F. Studer, and M. Toulemonde, Track formation in $\mathrm{SiO}_{2}$ quartz and the thermal-spike mechanism, Phys. Rev. B 49(18), 12457 (1994).

[Men97] V. Mennella, G. A. Baratta, L. Colangeli, P. Palumbo, A. Rotundi, E. Bussoletti, and G. Strazzulla, Ultraviolet Spectral Changes in Amorphous Carbon Grains Induced by Ion Irradiation, Astrophys J. 481, 545 (1997).

[Mol01] M. E. Toimil Molares, J. Brötz, V. Buschmann, D. Dobrev, R. Neumann, R. Scholz, I. U. Schuchert, C. Trautmann, and J. Vetter, Etched heavy ion tracks in polycarbonate as template for copper nanowires, Nucl. Instrum. Methods Phys. Res., Sect. B 185(1-4), 192 (2001).

[Nix05] A.-K. Nix, Elektrische Eigenschaften von graphitischen Ionenspuren in diamantartigen Kohlenstoffschichten, Master's thesis, Universität Göttingen (2005).

[Nor96] K. Nordlund, J. Keinonen, and T. Mattila, Formation of Ion Irradiation Induced Small-Scale Defects on Graphite Surfaces, Phys. Rev. Lett. 77, 699 (1996).

[Noy97] A. Noy, D. V. Vezenov, and C. M. Lieber, Chemical Force Mocroscopy, Annu. Rev. Mater Sci. 27, 381 (1997).

[Oth02] A. Othonos and C. Christofides, Ultrafast dynamics in phosphorusimplanted silicon wafers: The effects of annealing, Phys. Rev. B 66(8), 085206 (2002).

[Oza99] T. Ozaki, Bond-order potential based on the Lanczos basis, Phys. Rev. B 59(24), 16064 (1999).

[Pol76] M. Pollak and I. Riess, A percollation treatment of high-field hopping transport, J. Phys. C. 9, 2339 (1976).

[Ree83] R. C. Reedy, J. R. Arnold, and D. Lal, Cosmic-Ray Record in Solar System Matter, Science 219(4581), 127 (1983).

[Ron96] C. Ronning, Untersuchungen zum Wachstum und zur Dotierung diamantähnlicher Schichten, hergestellt über die Deposition massenseparierter Ionen, Ph.D. thesis, Universität Konstanz (1996). 
[Ron00] C. Ronning, R. Merk, H. Feldermann, F. Harbsmeier, and H. Hofsäss, Properties of ion beam deposited tetrahedral fluorinated amorphous carbon films ta-C:F, MRS Symp. Proc. 593 (2000).

[Ron03] C. Ronning, Ion-beam synthesis and growth mechanism of diamond-like materials, Appl. Phys. A 77(1), 39 (2003).

[Rot04] M. Roth, G. Schiwietz, K. Czerski, B. Schattat, F. Staufenbiel, and B. Walz, AIP Conference Proceedings, AIP Conf. Proc. 740, 175 (2004), pp. 175-181.

[Rub90] T. Diaz de la Rubia and M.W. Guinan, http://www. lınl.gov/ asci/platforms/purple/rfp/benchmarks/limited/mdcask/, Mat. Res. Forum 174, 151 (1990).

[Saa99] D. Saada, J. Adler, and R. Kalish, Computer simulation of damage in diamond due to ion impact and its annealing, Phys. Rev. B 59, 6650 (1999).

[Sal01] E Salonen, K. Nordlund, J. Keinonen, and C. H. Wu, Swift chemical sputtering of amorphous hydrogenated carbon, Phys. Rev. B 63, 195415 (2001).

[Sch99] G. Schiwietz et al., Determination of the electron temperature in the thermal spike of amorphous carbon, Europhys. Lett. 47(3), 384 (1999).

[Sch00] G. Schiwietz, G. Xiao, E. Luderer, and P. L. Grande, Auger electrons from ion tracks, Nucl. Instrum. Methods Phys. Res., Sect. B 164-165, 353 (2000).

[Sch04] D. Schwen, C. Ronning, and H. Hofsäss, Field emission studies on swift heavy ion irradiated tetrahedral amorphous carbon, Diamond Relat. Mater. 13, 1032 (2004).

[Sch06] D. Schwen and E. M. Bringa, Atomistic simulations of swift ion tracks in diamond and graphite, Nucl. Instrum. Methods Phys. Res., Sect. B (2006), in press, doi:10.1016/j.nimb.2006.12.001.

[Sig81] P. Sigmund and C. Claussen, Sputtering from elastic-collision spikes in heavy-ion-bombarded metals, J. Appl. Phys. 52, 990 (1981).

[Sil59] E. C. H. Silk and R. S. Barnes, Examination of Fission Fragment Tracks with an Electron Microscope, Phil. Mag. 8(4), 970 (1959). 
[Sko05] E. V. Skokan, I. V. Arkhangelskii, D. E. Izotov, N. V. Chelovskaya, M. M. Nikulin, and Yu. A. Velikodnyi, Stability of hexagonal modification of fullerite $C_{60}$, Carbon 43(4), 803 (2005).

[Smi90] R. Smith, A classical dynamics study of carbon bombardment of graphite and diamond, Proc. R. Soc. London, Ser. A 431, 143 (1990).

[Smi96] R. Smith and K. Beardmore, Molecular dynamics studies of particle impacts with carbon-based materials, Thin Solid Films 272, 255 (1996).

[Sor04] A. Sorkin, J. Adler, and R. Kalish, Computer simulations of damage due to passage of a heavy fast ion through diamond, Phys. Rev. B 70, 064110 (2004).

[Stu00] S. J. Stuart et al., A reactive potential for hydrocarbons with intermolecular interactions, J. Chem. Phys. 112, 6472 (2000).

[Stu06] S. J. Stuart, M. T. Kippenberg, O. Kum, and P. S. Krstic, Simulation of amorphous carbon with a bond-order potential, Physica Scripta T124, 58 (2006).

[Sud01] R. Sudowe, P. Vater, R. Brandt, J. Vetter, and W. Ensinger, Filters with $<100 \mathrm{~nm}$ radius pores for gas separation formed by high-energy ion irradiation of polymers, Nucl. Instrum. Methods Phys. Res., Sect. B 175, 564 (2001).

[Sze95] G. Szenes, General features of latent track formation in magnetic insulators irradiated with swift heavy ions, Phys. Rev. B 51(13), 8026 (1995).

[Sze97] G. Szenes, Amorphous track formation in $\mathrm{SiO}_{2}$, Nucl. Instrum. Methods Phys. Res., Sect. B 122, 530 (1997).

[Tad96] T. Tada and T. Kanayama, Nanolithography Using Fullerene Films as an Electron Beam Resist, Jpn. J. Appl. Phys. 35, L63 (1996).

[Ter83] J. Tersoff and D. R. Hamann, Theory and Application for the Scanning Tunneling Microscope, Phys. Rev. Lett. 50(25), 1998 (1983).

[Ter85] J. Tersoff and D. R. Hamann, Theory of the scanning tunneling microscope, Phys. Rev. B 31(2), 805 (1985).

[Ter88] J. Tersoff, Empirical Interatomic Potential for Carbon, with Applications to Amorphous Carbon, Phys. Rev. Lett. 61, 2879 (1988). 
[Tom94] T. A. Tombrello, Predicting latent track dimensions, Nucl. Instrum. Methods Phys. Res., Sect. B 94, 424 (1994).

[Tou92] M. Toulemonde, C. Dufour, and E. Paumier, Transient thermal process after a high-energy heavy-ion irradiation of amorphous metals and semiconductors, Phys. Rev. B 46(22), 14362 (1992).

[Tou02] M. Toulemonde, W. Assmann, C. Trautmann, and F. Grüner, Jetlike Component in Sputtering of LiF Induced by Swift Heavy Ions, Phys. Rev. Lett. 88, 057602 (2002).

[Tra00] C. Trautmann, M. Toulemonde, J. M. Costantini, J. J. Grob, and K. Schwartz, Swelling effects in lithium fluoride induced by swift heavy ions, Phys. Rev. B 62(1), 13 (2000).

[Tra06] C. Trautmann, personal communication (2006).

[Tro91] N. Troullier and J. L. Martins, Efficient pseudopotentials for plane-wave calculations, Phys. Rev. B 43(3), 1993 (1991).

[Tuc05] O. J. Tucker et al., Molecular dynamics simulation of sputtering from a cylindrical track: EAM versus pair potentials, Nucl. Instrum. Methods Phys. Res., Sect. B 228, 163 (2005).

[Ube04] B. Uberuaga et al., Structure and Mobility of Defects Formed from Collision Cascades in MgO, Phys. Rev. Lett. 92, 115505 (2004).

[Urb94] H. M. Urbassek, H. Kafemann, and R. E. Johnson, Atom ejection from a fast-ion track: A molecular-dynamics study, Phys. Rev. B 49, 786 (1994).

[Vin76] G. H. Vineyard, Thermal spikes and activated processes, Radiat. Eff. 29, 245 (1976).

[Vol02] A. E Volkov and V. A. Borodin, Effects of relaxation of electronic excitations from swift heavy ions in amorphous targets, Nucl. Instrum. Methods Phys. Res., Sect. B 193, 381 (2002).

[Vos05] M. Vos, C. Bowles, A. S. Kheifets, and M. R. Went, Electron momentum spectroscopy of light and heavy targets, J. Electron. Spectrosc. Relat. Phenom. 149, 20 (2005).

[Wal86] M. P. R. Waligorski, R. N. Hamm, and R. Katz, The radial distribution of dose around the path of a heavy ion in liquid water, Nucl. Tracks Radiat. Meas. 11, 309 (1986). 
[Wan94] Z. G. Wang, C. Dufour, E. Paumier, and M. Toulemonde, The $S_{e}$ sensitivity of metals under swift-heavy-ion irradiation: a transient thermal process, J. Phys.: Condens. Matter 6(34), 6733 (1994).

[You58] D. A. Young, Etching of Radiation Damage in Lithium Fluoride, Nature 182, 375 (1958).

[Zal83] R. Zallen, The Physics of Amorphous Solids, Wiley-Interscience, 1 edn. (1983).

[Zer42] F. Zernike, Phase Contrast: A New Method for the Microscopic Observation of Transparent Objects, Nijhoff (1942).

[Zie85] J. F. Ziegler, J. P. Biersack, and U. Littmark, The Stopping and Range of Ions in Solids, Pergamon Press, 1 edn. (1985). 


\section{Acknowledgements}

The author is deeply grateful for the help and support received by the following people:

- Prof. Dr. Hans Hofsäss, University of Göttingen, for all the freedom and support

- Dr. Eduardo Bringa, LLNL, Livermore, USA, for introduction to and fruitful discussions on the molecular dynamics simulations

- Dr. Carsten Ronning, University of Göttingen for the interesting work on nano structures and his motivational efforts

- Dr. Christina Trautmann, GSI, Darmstadt, for the swift heavy ion irradiations and discussions

- Prof. Dr. Hans Krauser, Hochschule Harz, Wernigerode for assistance and guidance with the AFM measurements

- Dr. Inga Gerhards and Dr. Tore Niermann for TEM preparations, imaging, and valuable discussions

- Isaac Tamblyn, Dalhousie University, Halifax, Canada for helpful discussions and computing time for some of the DFT calculations.

- Sebastian Loth for STM/STS measurements and valuable discussions

- Dr. Andrey Kamarou, Freidrich-Schiller-Universität Jena and Dr. Kay-Obbe Voss, GSI for additional irradiations at ISL/HMI, Berlin and CSNSM, Orsay.

- Helga Krüsemann and Dr. Katrin Lepler for orthographic and grammatical checking of the manuscript 



\title{
Curriculum Vitae
}

\author{
Daniel Schwen \\ Kellnerweg 14/220 \\ 37077 Göttingen \\ Tel.: +49 (0)5515033385 \\ E-Mail: dschwen@uni-goettingen.de \\ geboren 12.06.1977 in Hannover, Deutschland \\ deutscher Staatsangehöriger
}

Eltern Dominique Edovige Schwen-Fritschi, geb. Fritschi

geboren 25.05.1941 in Saint-Germain-en-Laye, Frankreich

Volkshochschuldozentin

Gustav Wilhelm Schwen

geboren 02.04.1927 in Emden, Deutschland

Diplom Bauingineur, pensioniert

\section{Ausbildung}

07.1981-08.1983 „Deutsches Rotes Kreuz“-Kindergarten, Wunstorf, Deutschland

09.1983-08.1987 Grundschule „am Bürgerpark“, Wunstorf, Deutschland

09.1987-08.1989 Orientierungsstufe „OS Nord“, Wunstorf, Deutschland

09.1989-07.1996 Gymnasialstufe „Hölty Gymnasium“, Wunstorf, Deutschland

10.1997-06.1999 Grundstudium, Physik, Georg-August-Universität Göttingen, Deutschland

07.1999-07.2000 Auslandsaufenthalt, University Of California Los Angeles (UCLA), Los Angeles, USA

08.2000-11.2002 Hauptstudium, Physik, Georg-August-Universität, Göttingen, Deutschland

06.2006-08.2006 Forschungsaufenthalt am Lawrence Livermore National Laboratory, USA, Molekulardynamik Simulationen von Ionenspuren in Kohlenstoffmaterialien

04.2001-10.2002 Diplomarbeit angefertigt in der Gruppe von Prof. Hans Hofsäss, Georg-August-Universität Göttingen, Deutschland

Thema: „Untersuchungen zur Feldemission von Ionenspuren in diamantähnlichem Kohlenstoff" 

August-Universität Göttingen, Deutschland

Thema: "Structural and electronic properties of swift heavy ion tracks in amorphous carbon"

Abschlüsse

07.1996

07.1999

11.2002

\section{Zivildienst}

08.1996-08.1997 Altenheim „Haus am Bürgerpark“, Wunstorf, Deutschland
Abitur, „Hölty Gymnasium“, Wunstorf, Deutschland (Note: 1.7)

Vordiplom, Georg-August-Universität, Göttingen, Deutschland (Note: sehr gut)

Diplom, Georg-August-Universität, Göttingen, Deutschland (Note: sehr gut)

Göttingen, 9. Januar 2007 